\title{
Synthesis and Electrogenerated Chemiluminescence of Donor-substituted Phenylethynylcoumarins
}

Arumugasamy Elangovan, Jui-Hsien Lin, Shu-Wen Yang, Hsien-Yi Hsu and Tong-Ing Ho*

Department of Chemistry, National Taiwan University, Taipei-106, Taiwan

hall@ntu.edu.tw

\section{Supporting Information}

Contents

Page No:

1. Structures and synthetic scheme

S2

2. Characterization data

S3-S4

3. $\quad{ }^{1} \mathrm{H}-N M R$ and ${ }^{13} \mathrm{C}-N M R$ spectra of $\mathbf{1 - 1 0}$

S5-S24

4. X-Ray crystal structure (ORTEP) of 4

S25

5. Table of crystal data and structure refinement for $\mathbf{4}$

S26

6. Bond lengths and angles from crystal structure of $\mathbf{4}$

$\mathrm{S} 27$

7. Crystal packing diagram showing $\pi$-interaction in $\mathbf{4}$

S28

8. $\quad$ CV graphs for $\mathbf{1 - 5}$

S29-S33

9. Raw ECL spectra for $\mathbf{1 - 5}$

S34-S38

10. CV graphs for 6-10

S39-S43

11. Raw ECL spectra for $\mathbf{6}, \mathbf{8}$ and $\mathbf{1 0}$

S44-S46

12. Computational details

S47

13. Ground state structures, energies and Cartesian coordinates for 1, 6, 5 and 10

S48-S53

14. HOMO and LUMO surfaces of 1

S54

15. HOMO and LUMO surfaces of 6

S55

16. HOMO and LUMO surfaces of $\mathbf{6}$ after planarization

S56

17. HOMO and LUMO surfaces of 5

S57

18. HOMO and LUMO surfaces of $\mathbf{1 0}$

S58

19. HOMO and LUMO surfaces of $\mathbf{1 0}$ after planarization

S59 


\section{Scheme 1}

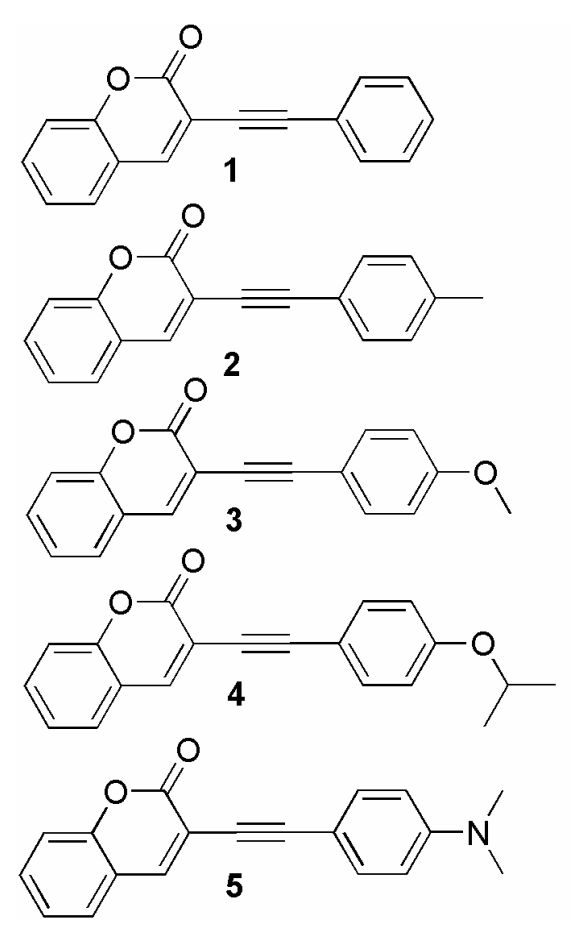

\section{Scheme 2}

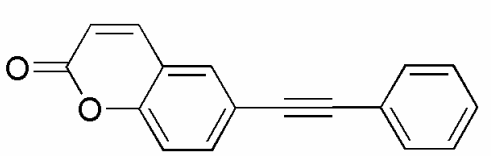

6
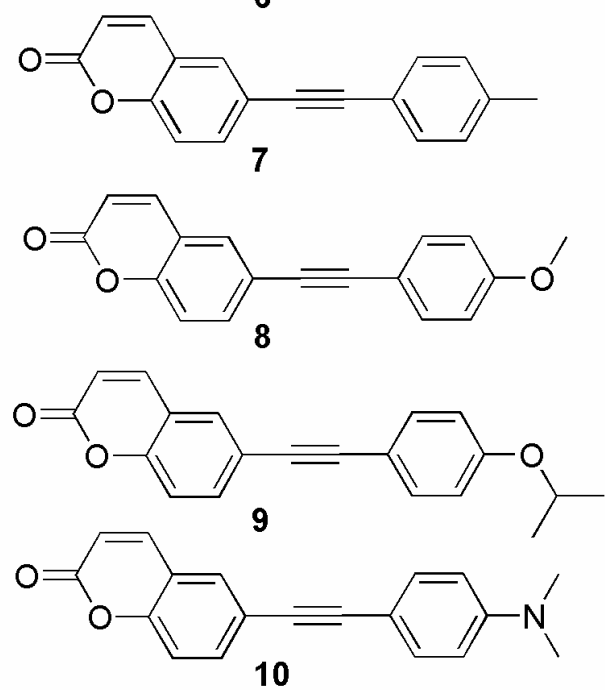

10

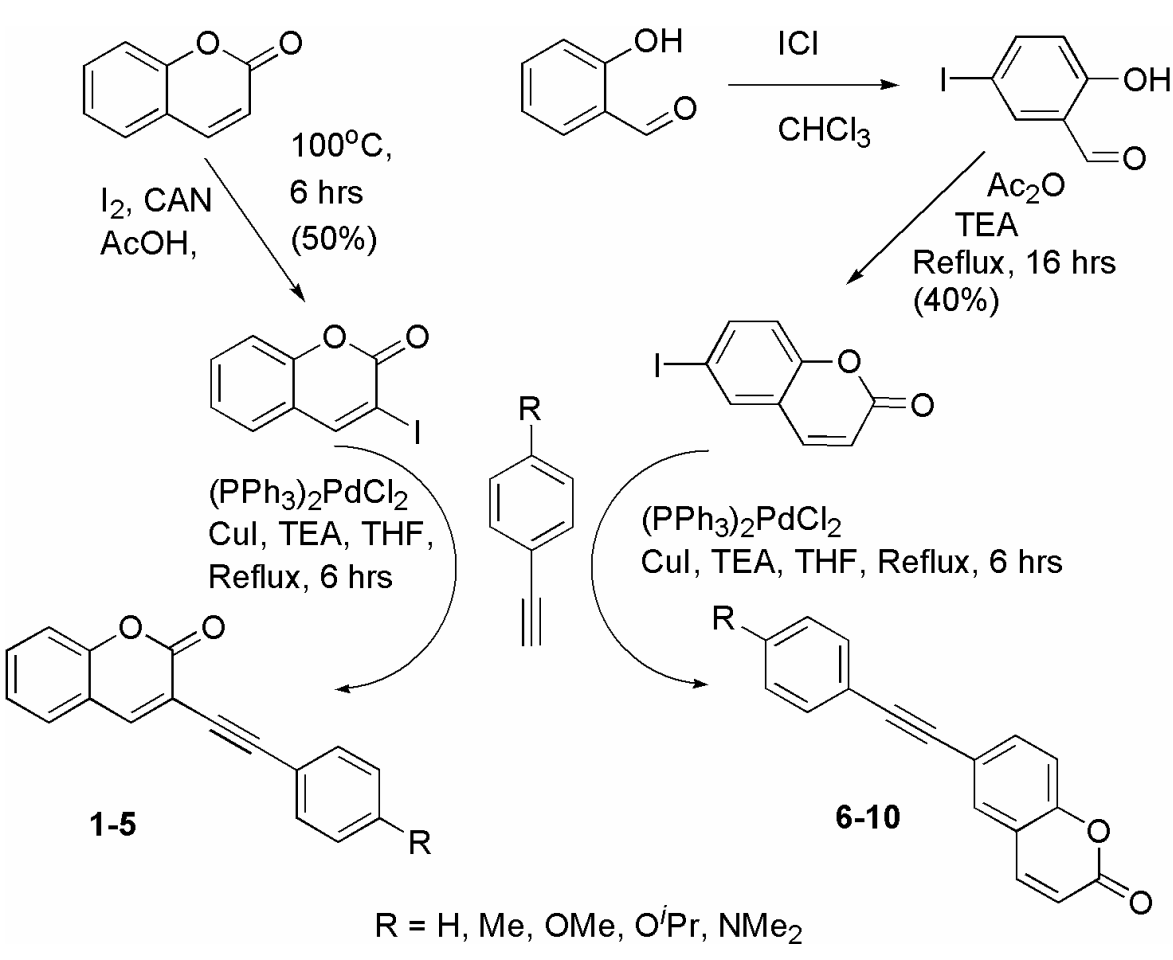

\section{Structures (Scheme 1) and synthetic scheme (Scheme 2) of preparation of donor-substituted phenylethynylcoumarins 1-10.}

Corrections to be incorporated in the paper jo0493424:

Structures of compounds 6-10 in Scheme 1 to appear as 6-isomers and not as 7-isomers and all terms reading 7-coumarin derivative(s) to read as 6-coumarin derivative(s). Any reference to compounds 6-10 in the text, figures and tables implies reference to the 6 -isomer and not to the 7 -isomer as incorrectly reported in the paper. 4-iodosalicylaldehyde to read as 5-iodosalicylaldehyde in the text.

The correct schemes are provided in this corrected Supporting Information that also carries the corrected structures in the spectra for compounds 6-10 and corrected results of the PM3 Calculations. PM3 calculations for the 6-isomers (compounds 6 and 10) revealed that the concept discussed in the result and discussion holds good. All the data including those given in the SI are maintainable for the corrected structures. All the results, discussion and conclusions still hold good and hence remain unaltered.

The error is regretted. 


\section{Characterization Data for 1 - 5}

\section{3-Phenylethynylcoumarin (1)}

From phenylacetylene and 3-iodocoumarin in $89 \%$ yield. M.p.: $176-177{ }^{\circ} \mathrm{C} ;{ }^{1} \mathrm{H}-\mathrm{NMR}\left(400 \mathrm{MHz}, \mathrm{CDCl}_{3} ; \delta, \mathrm{ppm}\right) 7.93(\mathrm{~s}$, 1H), 7.57-7.46 (m, 4H), 7.35-7.26 (m, 5H); ${ }^{13} \mathrm{C}-\mathrm{NMR}\left(100 \mathrm{MHz}, \mathrm{CDCl}_{3} ; \delta, \mathrm{ppm}\right) 159.1,153.1,144.5,132.0,131.8,128.9$, 128.2, 127.6, 124.7, 122.0, 118.8, 116.7, 113.0, 95.7, 83.3; HR-FAB M.S. m/z (M+): 246.0681, calculated for $\mathrm{C}_{17} \mathrm{H}_{10} \mathrm{O}_{2}$ : 246.0681 .

\section{3-p-Tolylethynylcoumarin (2)}

From p-tolylethyne and 3-iodocoumarin in $88 \%$ yield. M.p.: $127-128{ }^{\circ} \mathrm{C}$; ${ }^{1} \mathrm{H}$ NMR $(\delta, p p m): 7.91(\mathrm{~s}, 1 \mathrm{H}), 7.53-7.46(\mathrm{~m}$, 4H), 7.33-7.26 (m, 2H), $7.15(\mathrm{~d}, J=8 \mathrm{~Hz}, 2 \mathrm{H}), 2.36(\mathrm{~s}, 3 \mathrm{H}) ;{ }^{13} \mathrm{C}$ NMR $(\delta, \mathrm{ppm}): 159.17,153.01,144.2,139.3,131.8$, 131.7, 129.0, 127.5, 124.6, 118.9, 118.8, 116.6, 113.1, 96.1, 82.7, 21.7; MS (M+) m/z: 260.0838, calculated for $\mathrm{C}_{18} \mathrm{H}_{12} \mathrm{O}_{2}: 260.0837$.

\section{3-p-Anisylethynylcoumarin (3)}

From $p$-anisylethyne and 3-iodocoumarin in $85 \%$ yield. M.p.: $162-165{ }^{\circ} \mathrm{C} ;{ }^{1} \mathrm{H}-\mathrm{NMR}(\delta, \mathrm{ppm}) 7.89(\mathrm{~s}, 1 \mathrm{H}), 7.52-7.44(\mathrm{~m}$, 4H), 7.33-7.25 (m, 2H), $6.86(\mathrm{~d}, \mathrm{~J}=8.8 \mathrm{~Hz}, 2 \mathrm{H}), 3.81(\mathrm{~s}, 3 \mathrm{H}) ;{ }^{13} \mathrm{C}-\mathrm{NMR}(\delta, \mathrm{ppm}): 160.0,129.2,152.9,143.8,133.4$, 131.7, 127.4, 124.6, 118.9, 116.6, 114.1, 113.9, 113.2, 96.1, 82.2, 55.4; HR-FAB M.S. m/z (M+): 276.0790, calculated for $\mathrm{C}_{18} \mathrm{H}_{12} \mathrm{O}_{3}: 276.0786$.

\section{3-(p-O-Isopropyl)phenylethynylcoumarin (4)}

From 4-O-isopropylphenylethyne and 3-iodocoumarin in $73 \%$ yield. M.p.: $147-148{ }^{\circ} \mathrm{C} ;{ }^{1} \mathrm{H}-\mathrm{NMR}(\delta, \mathrm{ppm}) 7.88(\mathrm{~s}, 1 \mathrm{H})$, 7.52-7.45 (m, 4H), 7.33-7.25 (m, 2H), $6.83(\mathrm{~d}, J=8.8 \mathrm{~Hz}, 2 \mathrm{H}), 4.57$ (septet, $J=6.4 \mathrm{~Hz}, 1 \mathrm{H}), 1.33(\mathrm{~d}, J=6.4 \mathrm{~Hz}, 6 \mathrm{H})$; ${ }^{13} \mathrm{C}-N M R(\delta \mathrm{ppm}) 159.3,158.5,152.9,143.7,133.5,131.7,127.4,124.6,118.9,116.7,115.6,113.7,113.4,96.3,82.1$, 69.9, 22.1; HR-FAB M.S. m/z (M+): 304.1095, calculated for $\mathrm{C}_{20} \mathrm{H}_{16} \mathrm{O}_{3}: 304.1099$.

\section{3-(p-N,N-Dimethylamino)phenylethynylcoumarin (5)}

From $N, N$-dimethylaminophenylethyne and 3-iodocoumarin in $88 \%$ yield. M.p.; $205-206{ }^{\circ} \mathrm{C} ;{ }^{1} \mathrm{H}-\mathrm{NMR}(\delta, \mathrm{ppm}) 7.84(\mathrm{~s}$, $1 \mathrm{H}), 7.50-7.43(\mathrm{~m}, 4 \mathrm{H}), 7.32-7.26(\mathrm{~m}, 2 \mathrm{H}), 6.62(\mathrm{~d}, \mathrm{~J}=8.8 \mathrm{~Hz}, 2 \mathrm{H}), 2.99(\mathrm{~s}, 6 \mathrm{H}) ;{ }^{13} \mathrm{C}-\mathrm{NMR}(\delta, \mathrm{ppm}) 159.4,152.8,150.4$, 142.6, 133.1, 131.3, 127.2, 124.5, 119.2, 116.6, 113.8, 111.5, 108.6, 97.9, 81.9, 40.2; HR-FAB M.S.m/z (M+): 289.1098, calculated for $\mathrm{C} 19 \mathrm{H} 15 \mathrm{NO} 2: 289.1103$. 


\section{Characterization Data for 6 - 10}

\section{6-Phenylethynylcoumarin (6)}

From phenylacetylene and 6-iodocoumarin in $86 \%$ yield. M.p.: 74-76 ${ }^{\circ} \mathrm{C}$; ${ }^{1} \mathrm{H}-\mathrm{NMR}(\delta, \mathrm{ppm}) 7.65-7.67(\mathrm{~m}, 2 \mathrm{H}), 7.62$ $(\mathrm{s}, 1 \mathrm{H}), 7.51-7.49(\mathrm{~m}, 2 \mathrm{H}), 7.36(\mathrm{~d}, J=4.4 \mathrm{~Hz}, 2 \mathrm{H}), 7.34-7.20(\mathrm{~m}, 2 \mathrm{H}), 6.3(\mathrm{~d}, J=9.6 \mathrm{~Hz}, 1 \mathrm{H}) ;{ }^{13} \mathrm{C}-\mathrm{NMR}(\delta, \mathrm{ppm}) 160.0$, 153.2, 142.6, 134.6, 131.4, 130.6, 128.5, 128.2, 122.4, 119.6, 118.7, 117.1, 116.9, 89.9, 87.4; HR-FAB M.S. m/z (M+): 246.0682, calculated for $\mathrm{C}_{17} \mathrm{H}_{10} \mathrm{O}_{2}: 246.0714$.

\section{6-p-Tolylethynylcoumarin (7)}

From $p$-tolylacetylene and 6-iodocoumarin in $90 \%$ yield. M.p.: $183-186{ }^{\circ} \mathrm{C} ;{ }^{1} \mathrm{H}-\mathrm{NMR}(\delta, \mathrm{ppm}) 7.67(\mathrm{~s}, 1 \mathrm{H}), 7.65-7.63(\mathrm{~m}$, $2 \mathrm{H}), 7.41(\mathrm{~d}, J=7.6 \mathrm{~Hz}, 2 \mathrm{H}), 7.30(\mathrm{~d}, J=9.2 \mathrm{~Hz}, 1 \mathrm{H}), 7.15(\mathrm{~d}, J=7.6 \mathrm{~Hz}, 2 \mathrm{H}), 6.44(\mathrm{~d}, J=9.2 \mathrm{~Hz}, 1 \mathrm{H}), 2.36(\mathrm{~s}, 3 \mathrm{H}) ;{ }^{13} \mathrm{C}-$ $\operatorname{NMR}(\delta, \mathrm{ppm}) 160.0,153.1,142.6,138.7,134.6,131.3,130.5,129.0,119.6,119.3,118.6,117.1,116.9,90.1,86.8,21.6$; HR-FAB M.S.m/z (M+): 260.0839, calculated for $\mathrm{C}_{18} \mathrm{H}_{12} \mathrm{O}_{2}: 260.0837$.

\section{6-p-Anisylethynylcoumarin (8)}

From $p$-anisylethyne and 6-iodocoumarin in $85 \%$ yield. M.p.: $102-107{ }^{\circ} \mathrm{C} ;{ }^{1} \mathrm{H}-\mathrm{NMR}(\delta, \mathrm{ppm}) 7.63-7.67(\mathrm{~m}, 2 \mathrm{H}), 7.61(\mathrm{~s}$, $1 \mathrm{H}), 7.45(\mathrm{~d}, J=8.4 \mathrm{~Hz}, 2 \mathrm{H}), 7.28(\mathrm{~d}, J=8.0 \mathrm{~Hz}, 1 \mathrm{H}), 6.87(\mathrm{~d}, J=8.8 \mathrm{~Hz}, 2 \mathrm{H}), 6.43(\mathrm{~d}, J=8.2 \mathrm{~Hz}, 1 \mathrm{H}), 3.82(\mathrm{~s}, 3 \mathrm{H}) ;{ }^{13} \mathrm{C}-$ $\operatorname{NMR}(\delta, \mathrm{ppm}) 160.1,159.6,153.1,142.7,134.6,132.9,130.4,120.1,118.7,117.2,117.0,114.5,113.9,90.1,86.2,55.4$; HR-FAB M.S.m/z (M+): 276.0789, calculated for $\mathrm{C}_{18} \mathrm{H}_{12} \mathrm{O}_{3}: 277.0786$.

\section{6-(4-O-Isopropyl)phenylethynylcoumarin (9)}

From 4-O-isopropylphenylethyne and 6-iodocoumarin in $85 \%$ yield. M.p.: $112-116{ }^{\circ} \mathrm{C} ;{ }^{1} \mathrm{H}-\mathrm{NMR}(\delta \mathrm{ppm}) 7.66(\mathrm{~d}, \mathrm{~J}=6.8$ $\mathrm{Hz}, 1 \mathrm{H}), 7.60(\mathrm{~s}, 1 \mathrm{H}), 7.42(\mathrm{~d}, J=8.8 \mathrm{~Hz}), 7.27(\mathrm{~d}, J=8.4 \mathrm{~Hz}, \mathrm{H}), 6.84(\mathrm{~d}, J=8.2 \mathrm{~Hz}, 2 \mathrm{H}), 6.42(\mathrm{~d}, J=8.8 \mathrm{~Hz}, 1 \mathrm{H}), 4.55(\mathrm{q}$, $J=5.2 \mathrm{~Hz}, 1 \mathrm{H}), 1.33(\mathrm{~d}, J=6.0 \mathrm{~Hz}, 6 \mathrm{H}) ;{ }^{13} \mathrm{C}-\mathrm{NMR}(\delta \mathrm{ppm}) 160.0,158.0,153.0,142.7,134.5,132.9,130.4,120.1,118.7$, 117.1, 116.9, 115.6, 114.1, 90.2, 86.1, 69.9, 22.0; HR-FAB M.S.m/z (M+): 304.1103, calculated for $\mathrm{C}_{20} \mathrm{H}_{16} \mathrm{O}_{3}: 304.1099$.

\section{6-(4-N,N-Dimethylamino)phenylethynylcoumarin (10)}

From $\mathrm{N}, \mathrm{N}$-dimethylaminophenylethyne and 6-iodocoumarin in $92 \%$ yield. M.p.: $125-129{ }^{\circ} \mathrm{C} ; 1 \mathrm{H}-\mathrm{NMR}(\delta, \mathrm{ppm}) 7.65(\mathrm{~d}, \mathrm{~J}=$ $9.6 \mathrm{~Hz}, 2 \mathrm{H}), 7.56(\mathrm{~s}, 1 \mathrm{H}), 7.38(\mathrm{~d}, J=8.8 \mathrm{~Hz}, 2 \mathrm{H}), 7.26(\mathrm{~d}, J=8.4 \mathrm{~Hz}, 1 \mathrm{H}), 6.66(\mathrm{~d}, J=9.2 \mathrm{~Hz}, 2 \mathrm{H}), 6.42(\mathrm{~d}, J=9.6 \mathrm{~Hz}$, 1H), 2.98 (s, 6H); ${ }^{13} \mathrm{C}-\mathrm{NMR}(\delta, \mathrm{ppm}): 160.2,152.8,150.6,142.8,134.4,132.6,130.1,120.7,118.7,117.1,116.9,111.7$, 109.0, 91.4, 85.6, 40.2; HR-FAB M.S.m/z (M+): 289.1096, calculated for $\mathrm{C}_{20} \mathrm{H}_{16} \mathrm{O}_{3}: 289.1103$. 
RHL $-5-3$

Purse Sequence: s2pul

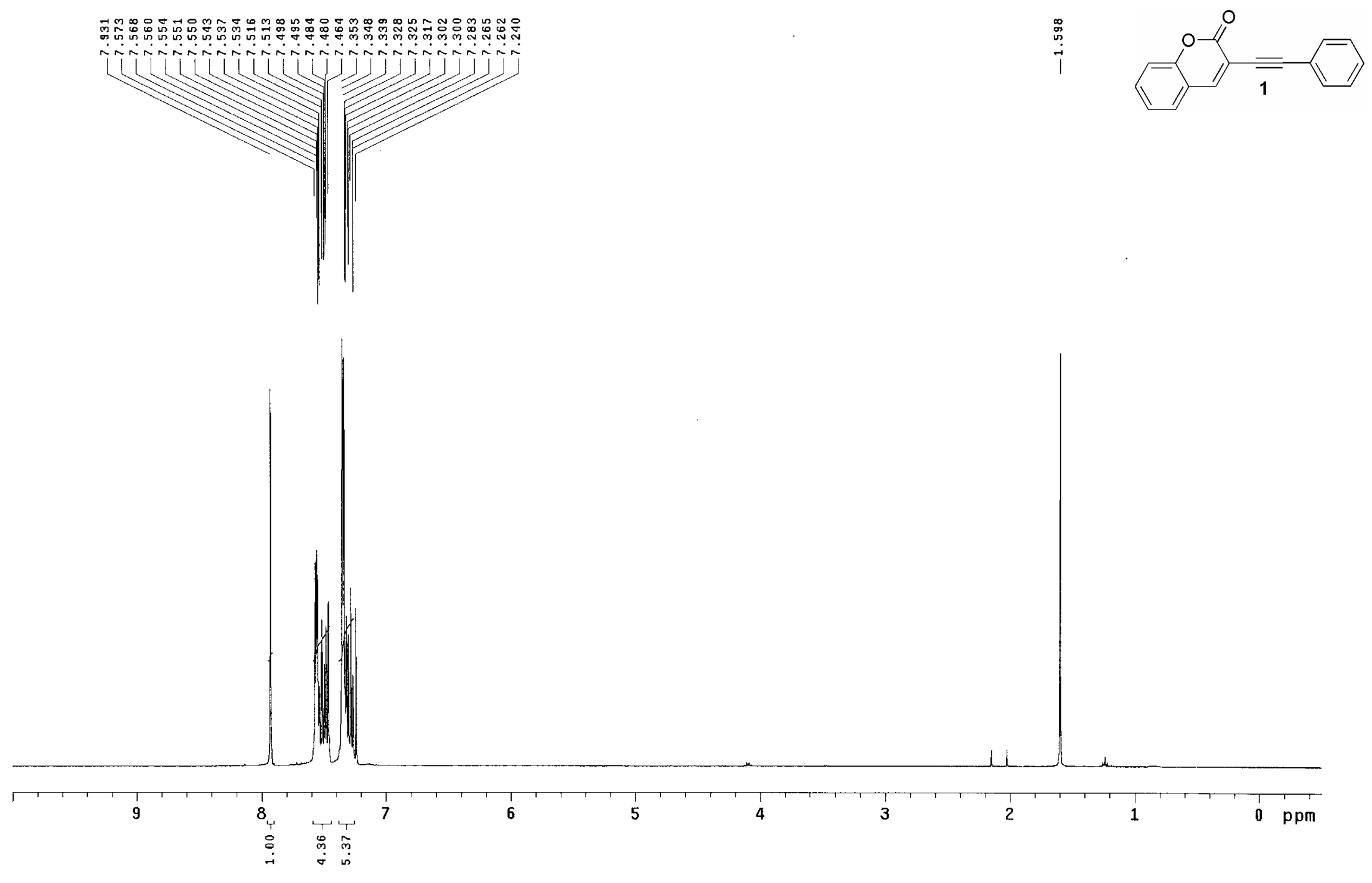


RHL-5-3

Pulse Sequence: $s 2 \mathrm{pur}$
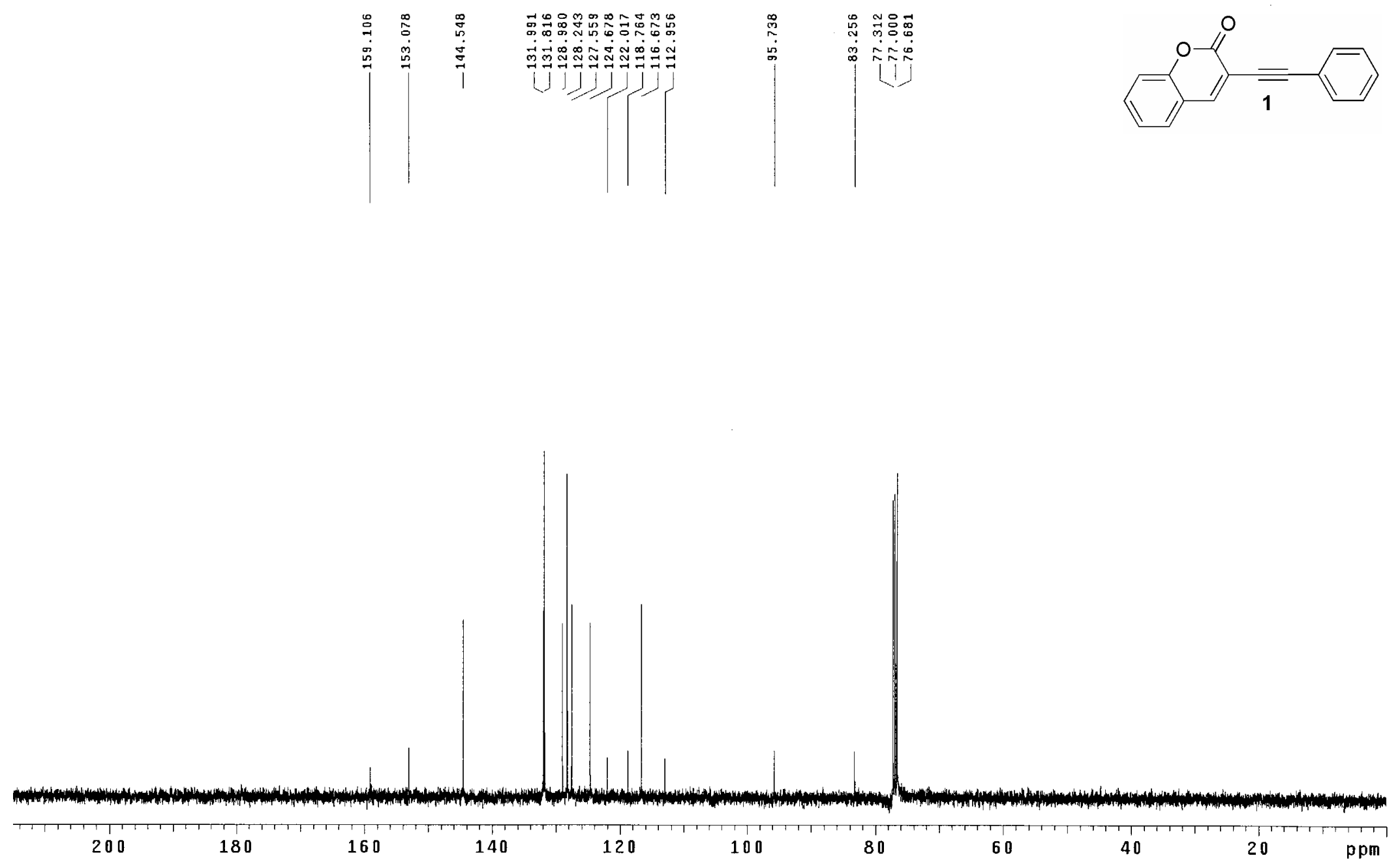
RHL - 5-1

Putse Sequence: s2pul
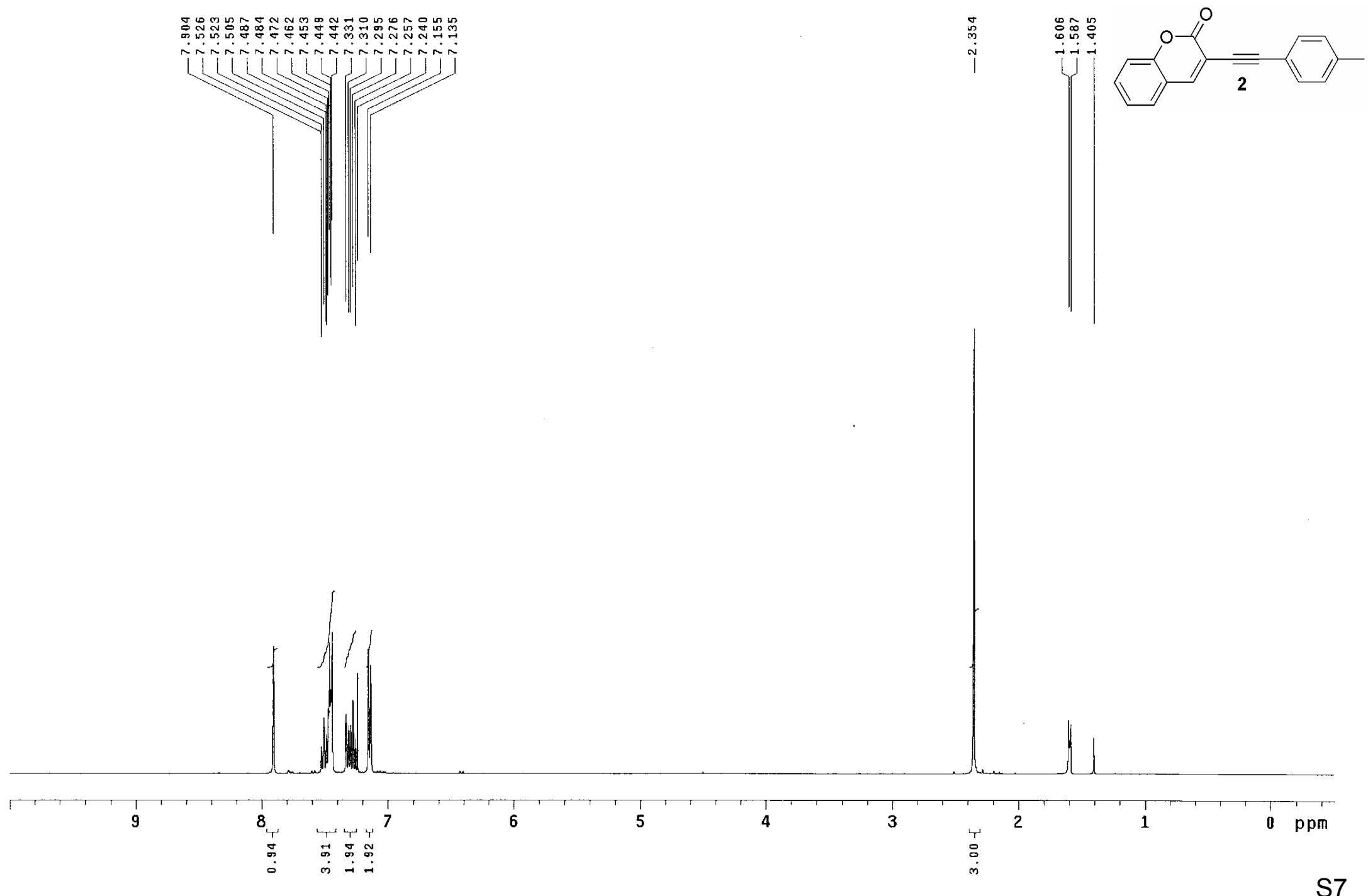
RHL -5-1

Pulse Sequence: s2pul
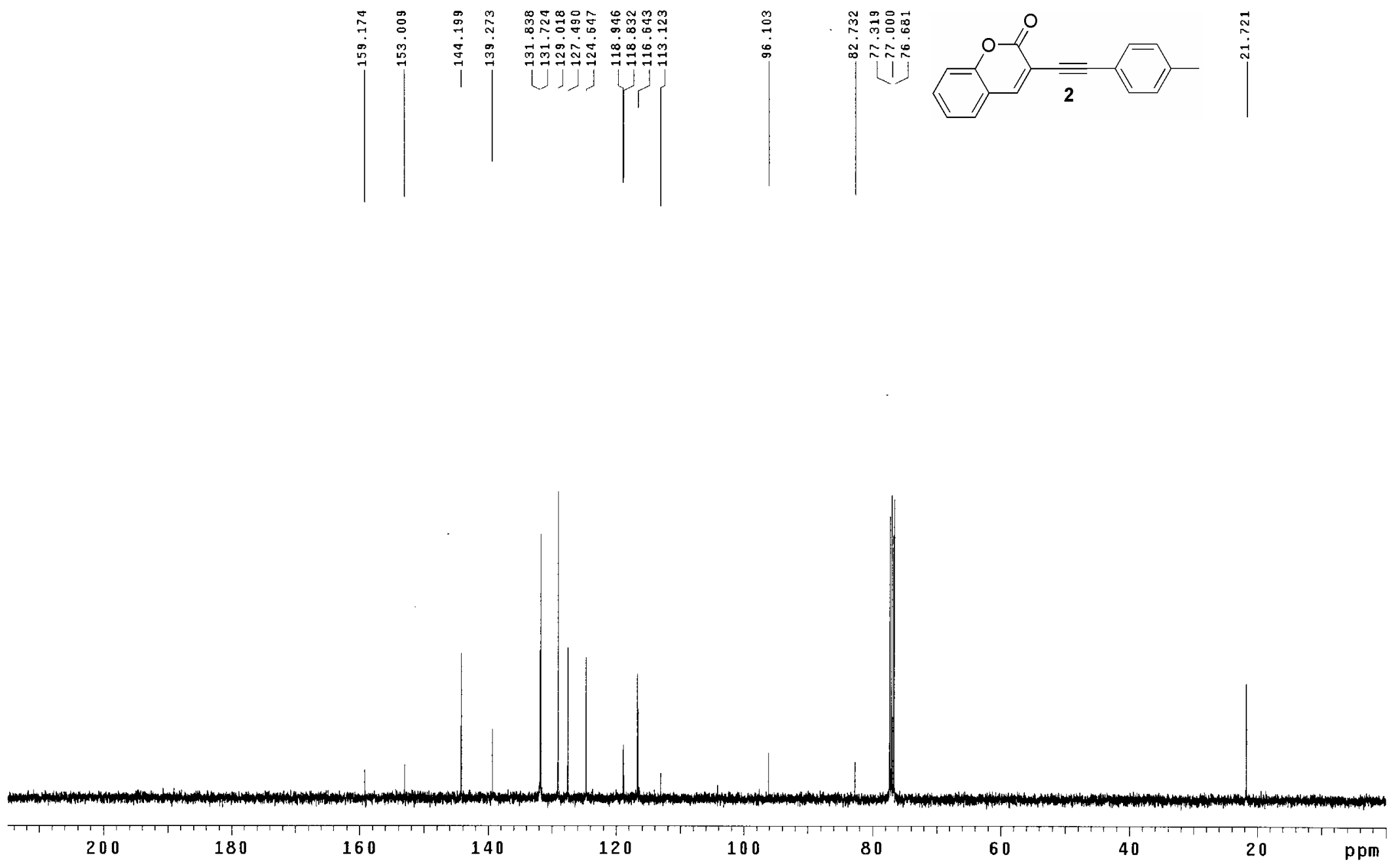
RHL -5-2

Pulse Sequence: $52 p u l$

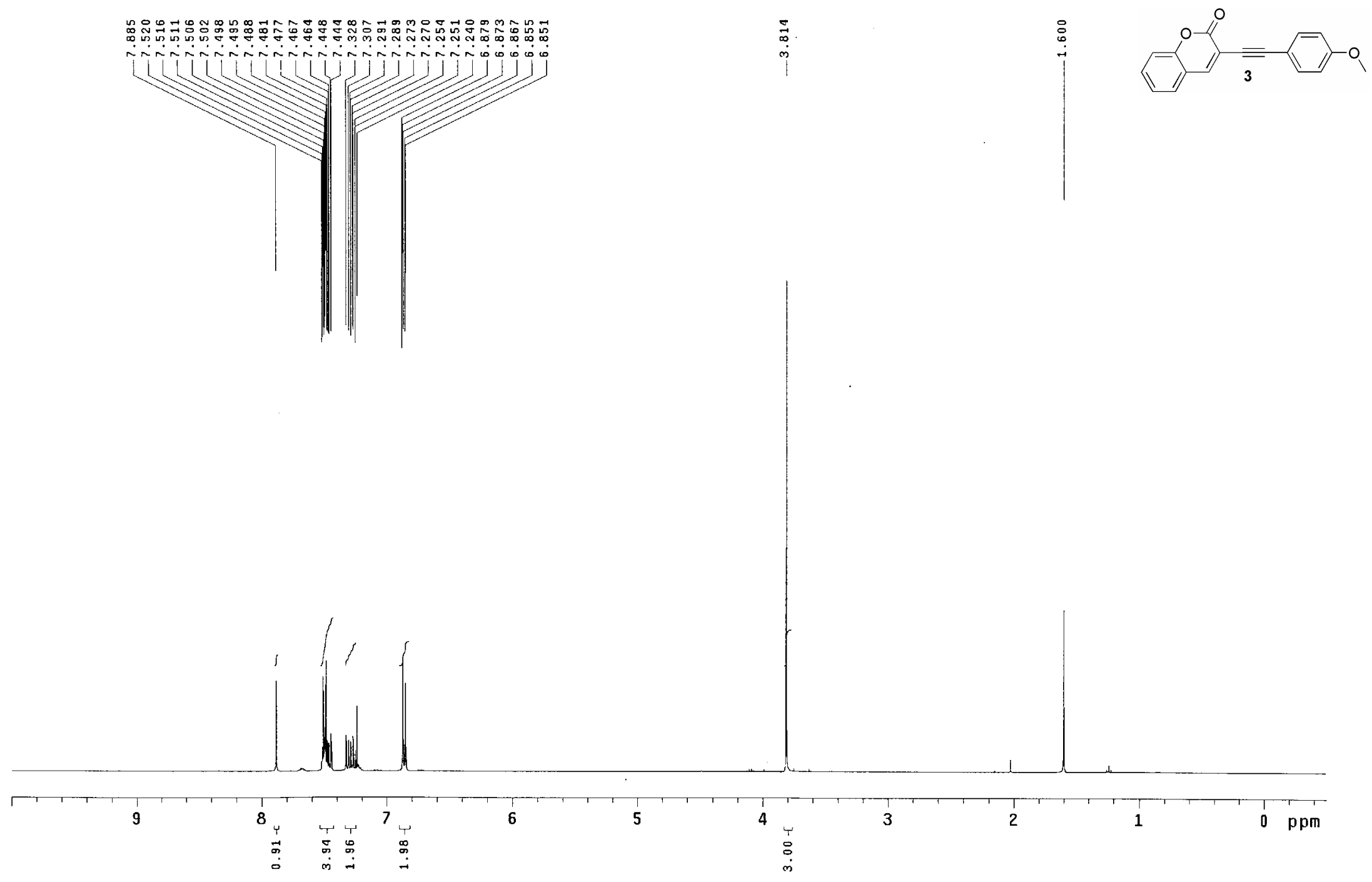


RHL-5-2

Putse Sequence: s2put
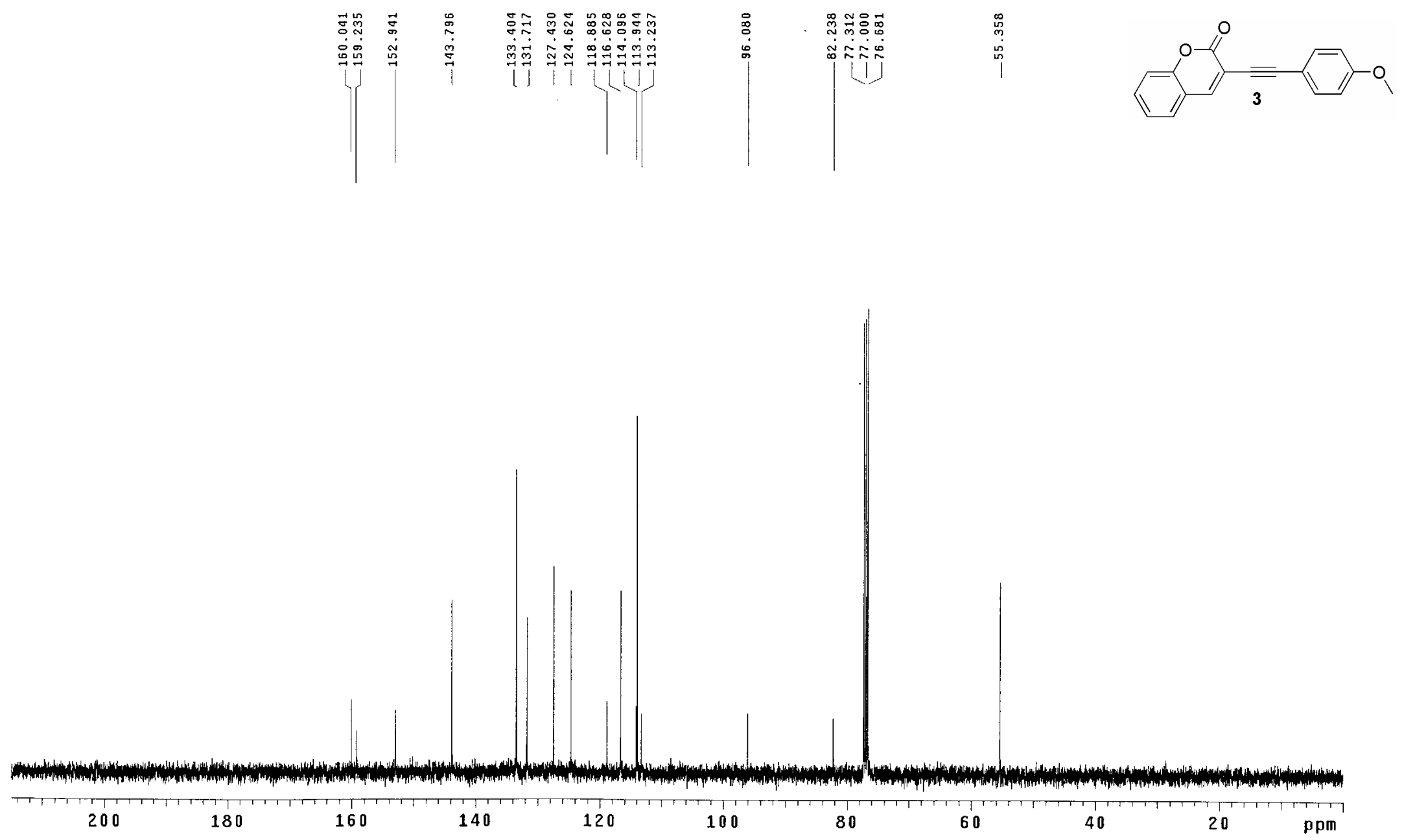
RHL -5-5

Pulse Sequence: $s 2 p u l$
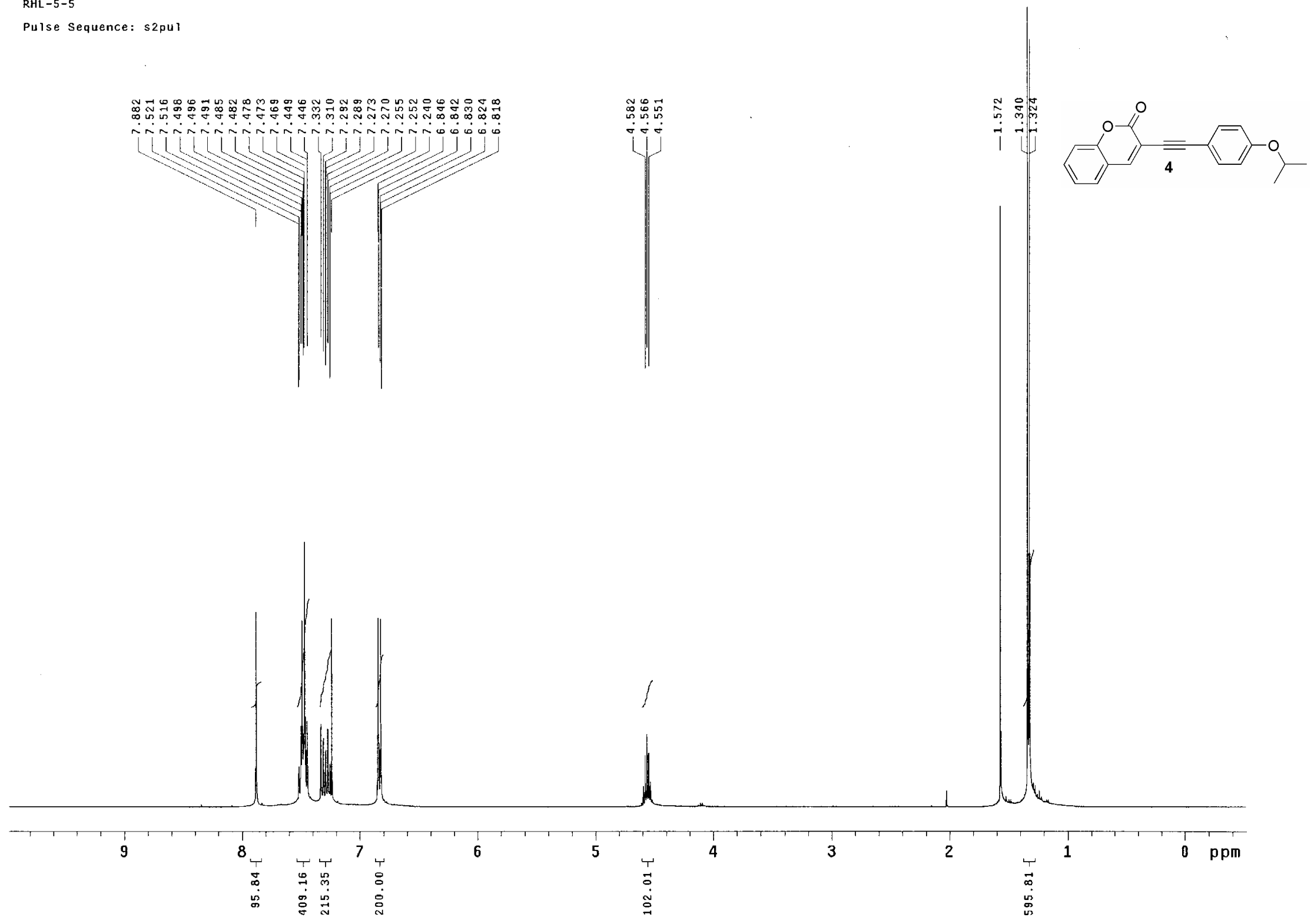
RHL $-5-5$

Pulse Sequence: s2pul

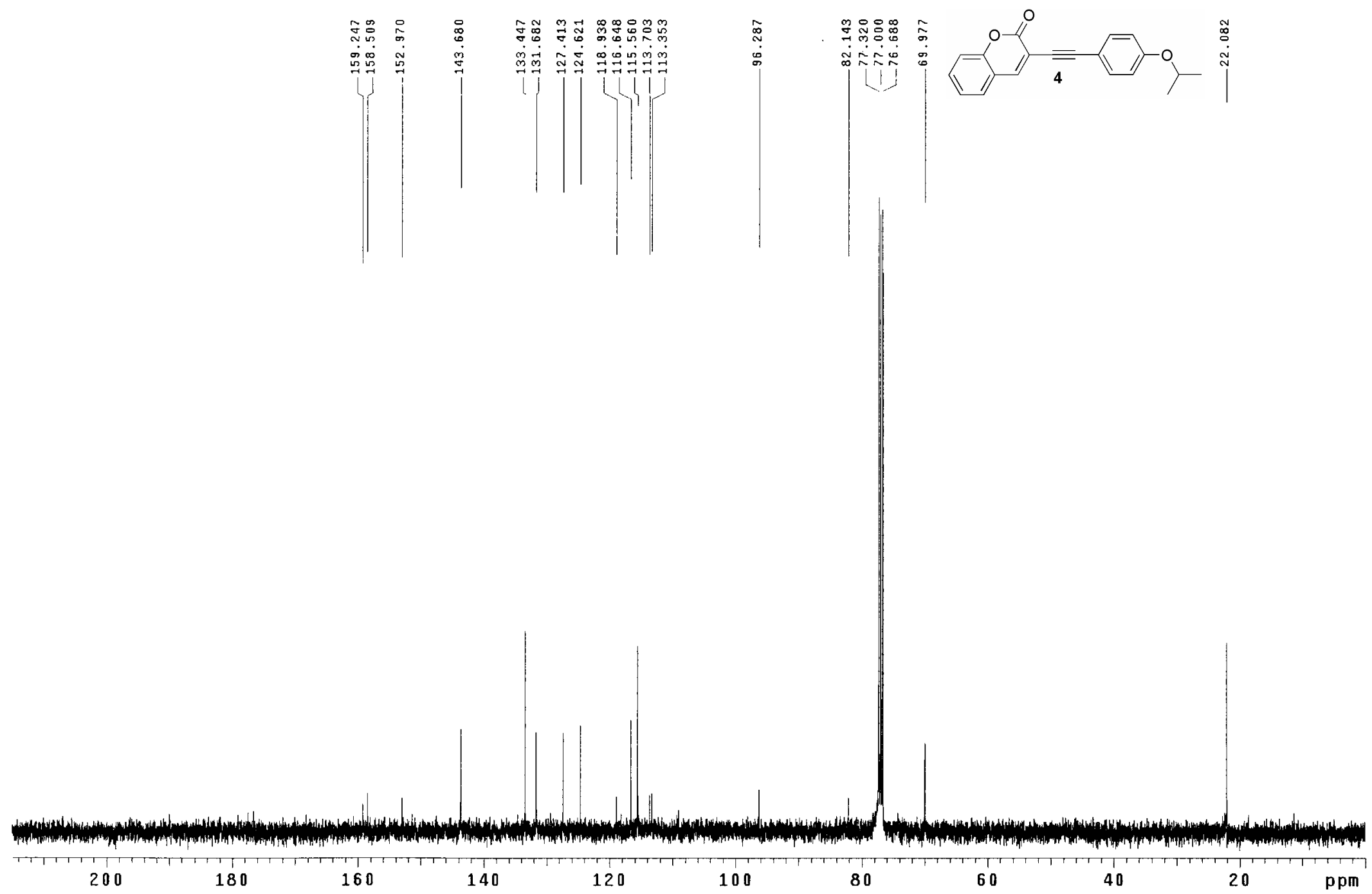


RHL -5-4

Pulse Sequence: s2pul

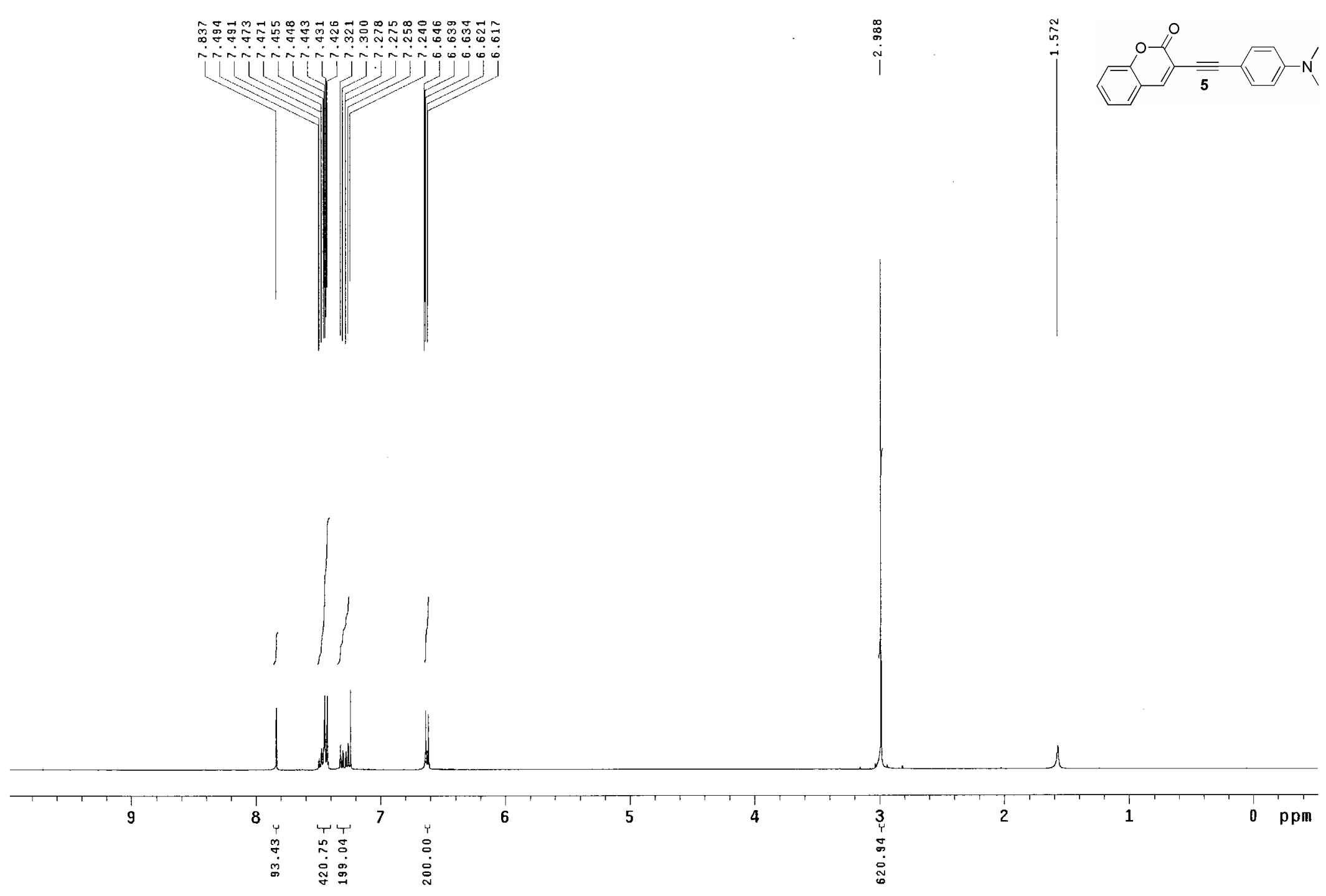


RHL-5-4

Pulse sequence: s2pur
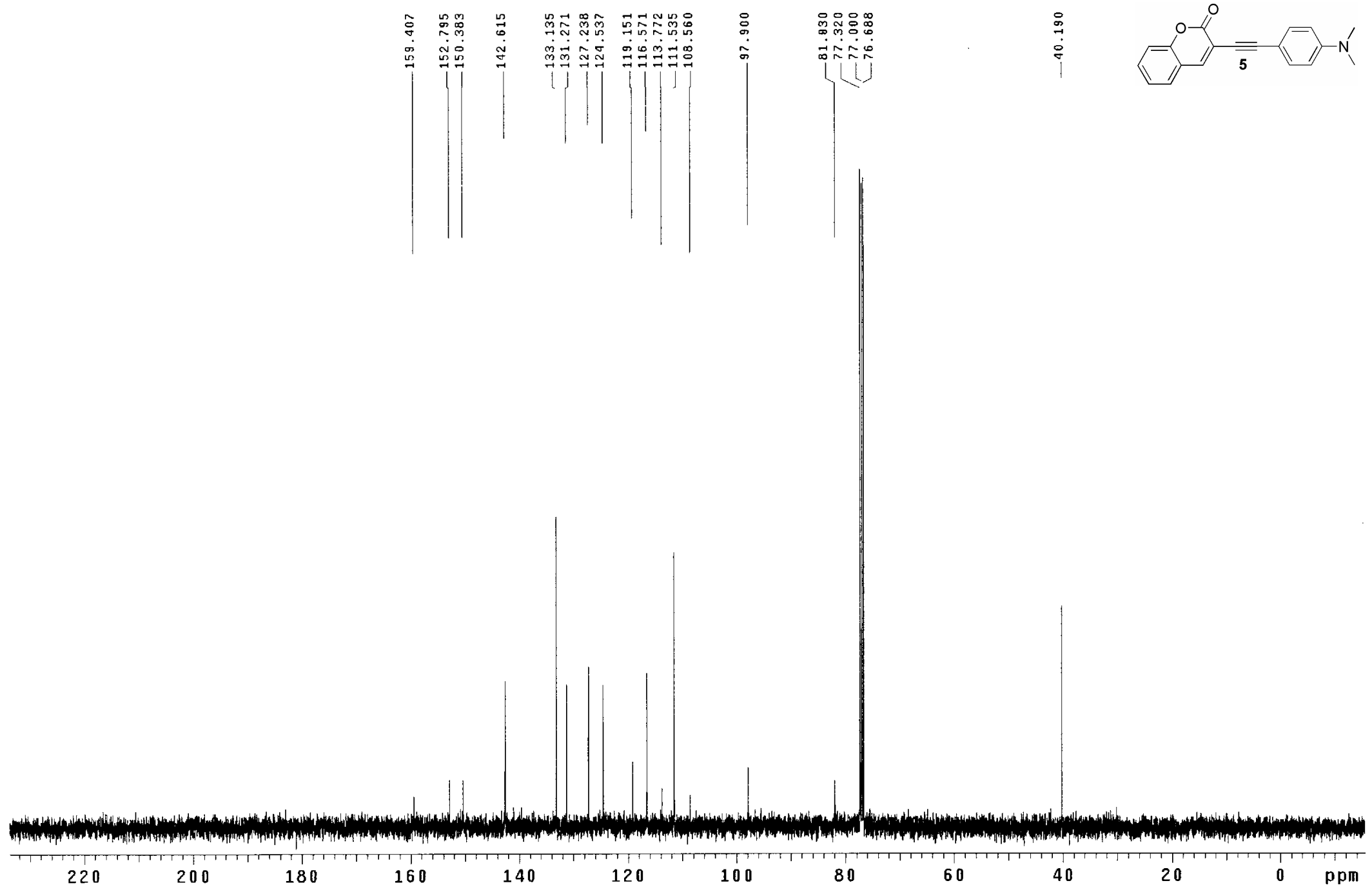

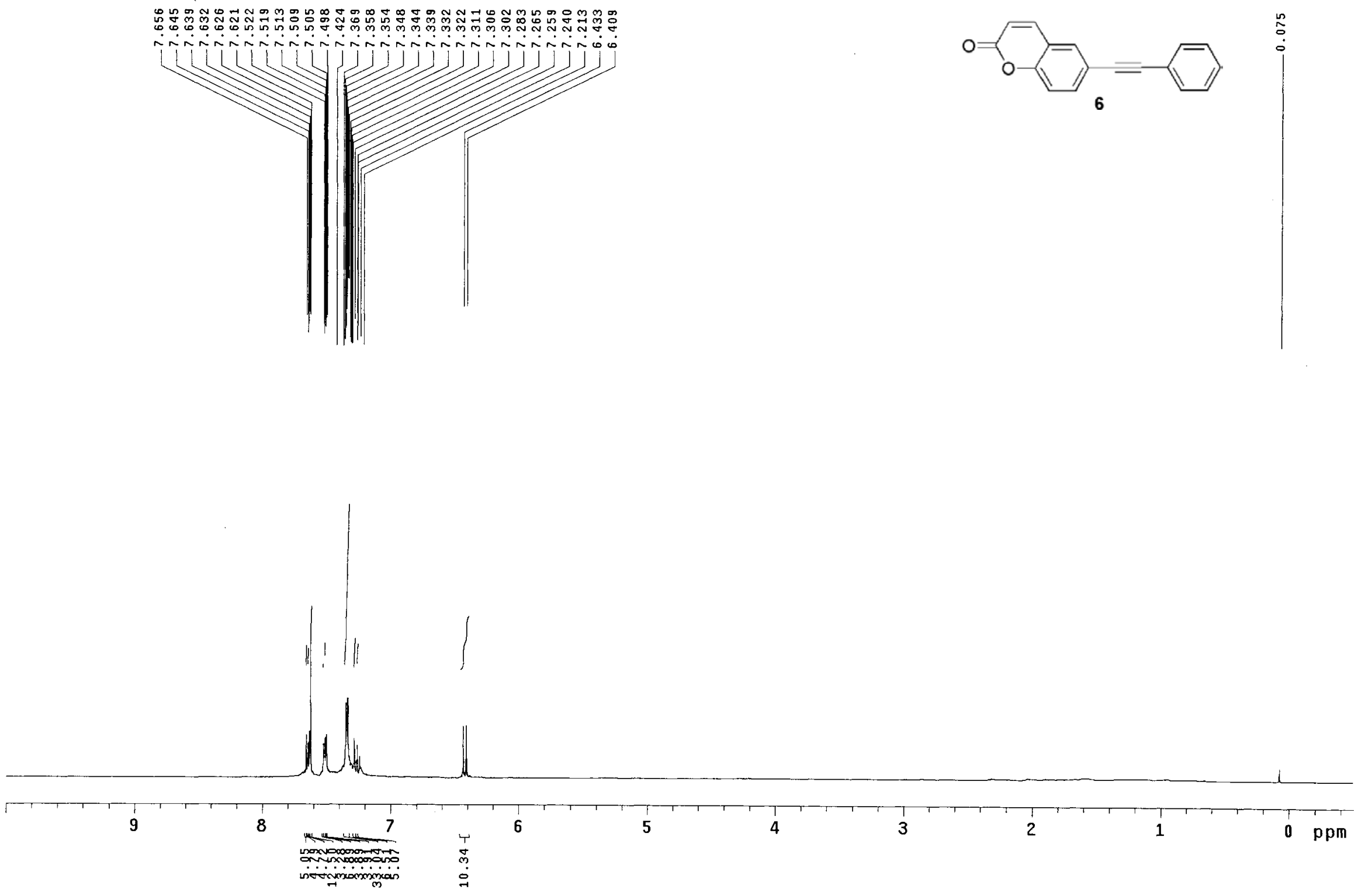

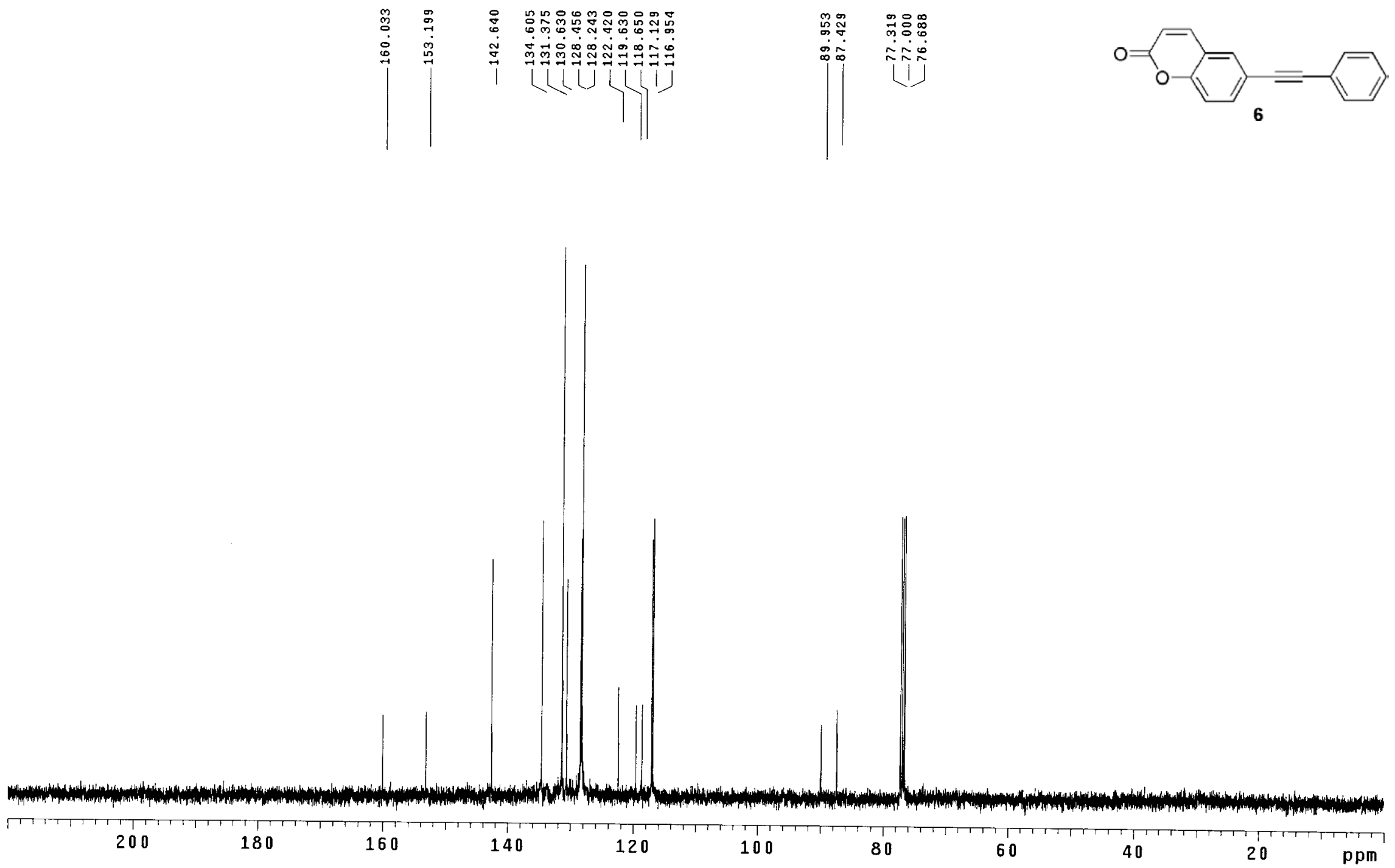


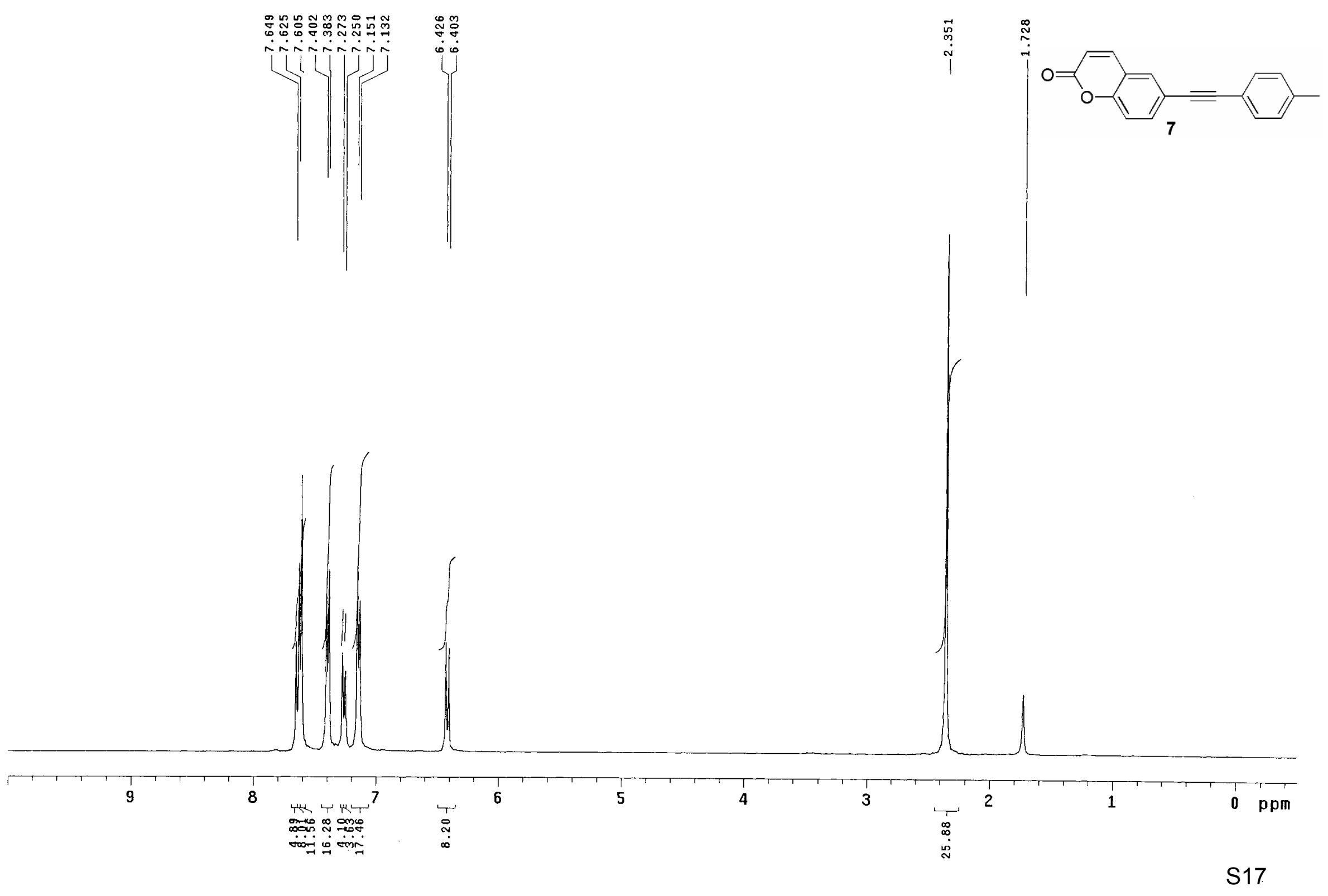



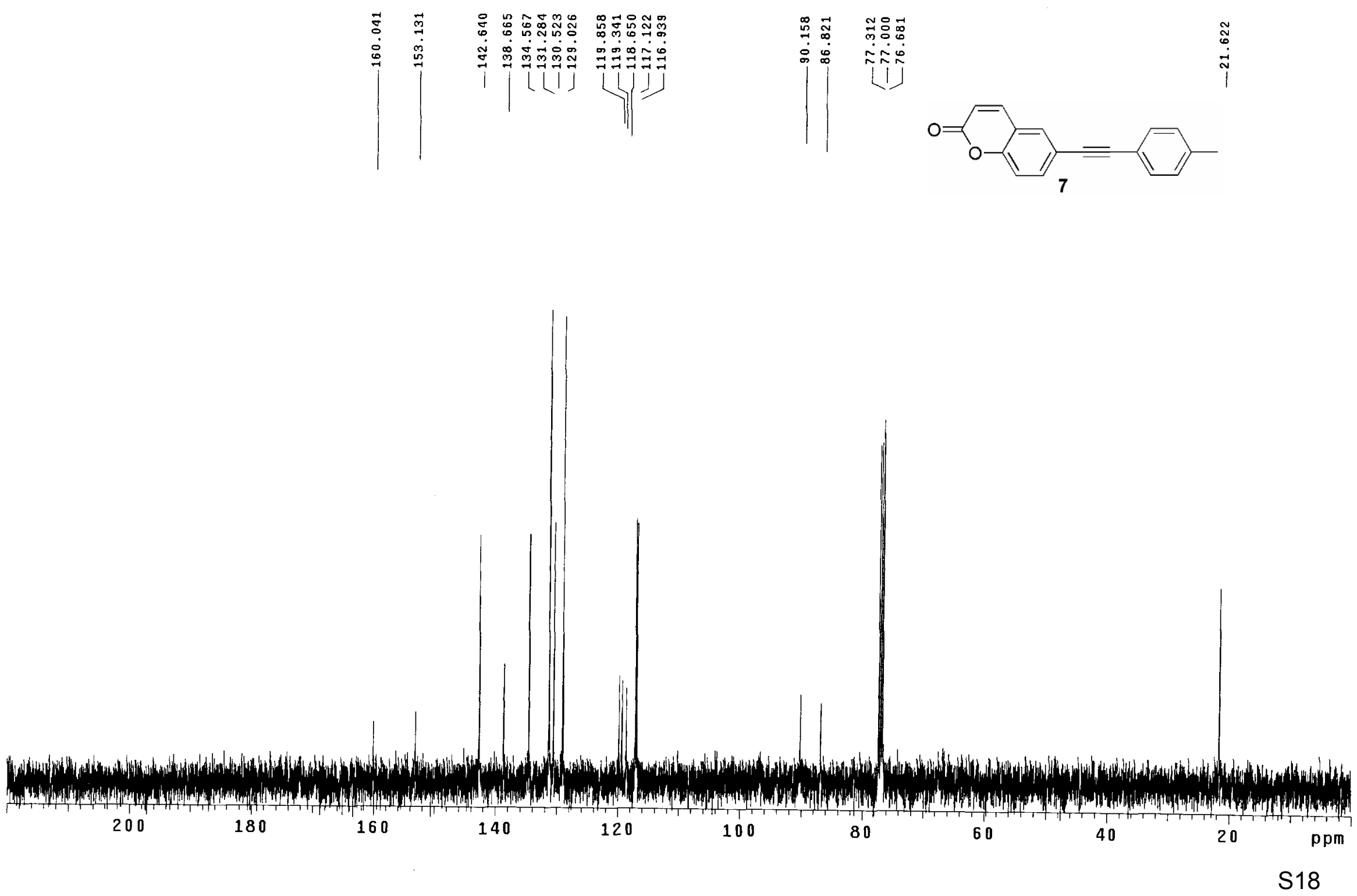


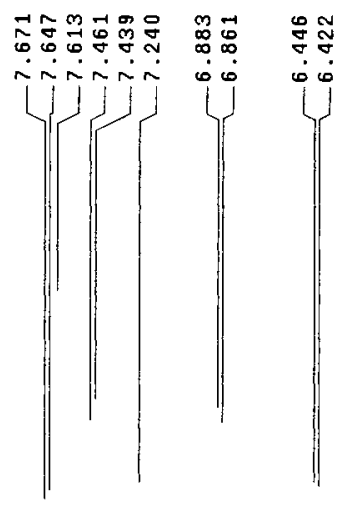

$\stackrel{\infty}{\infty}$
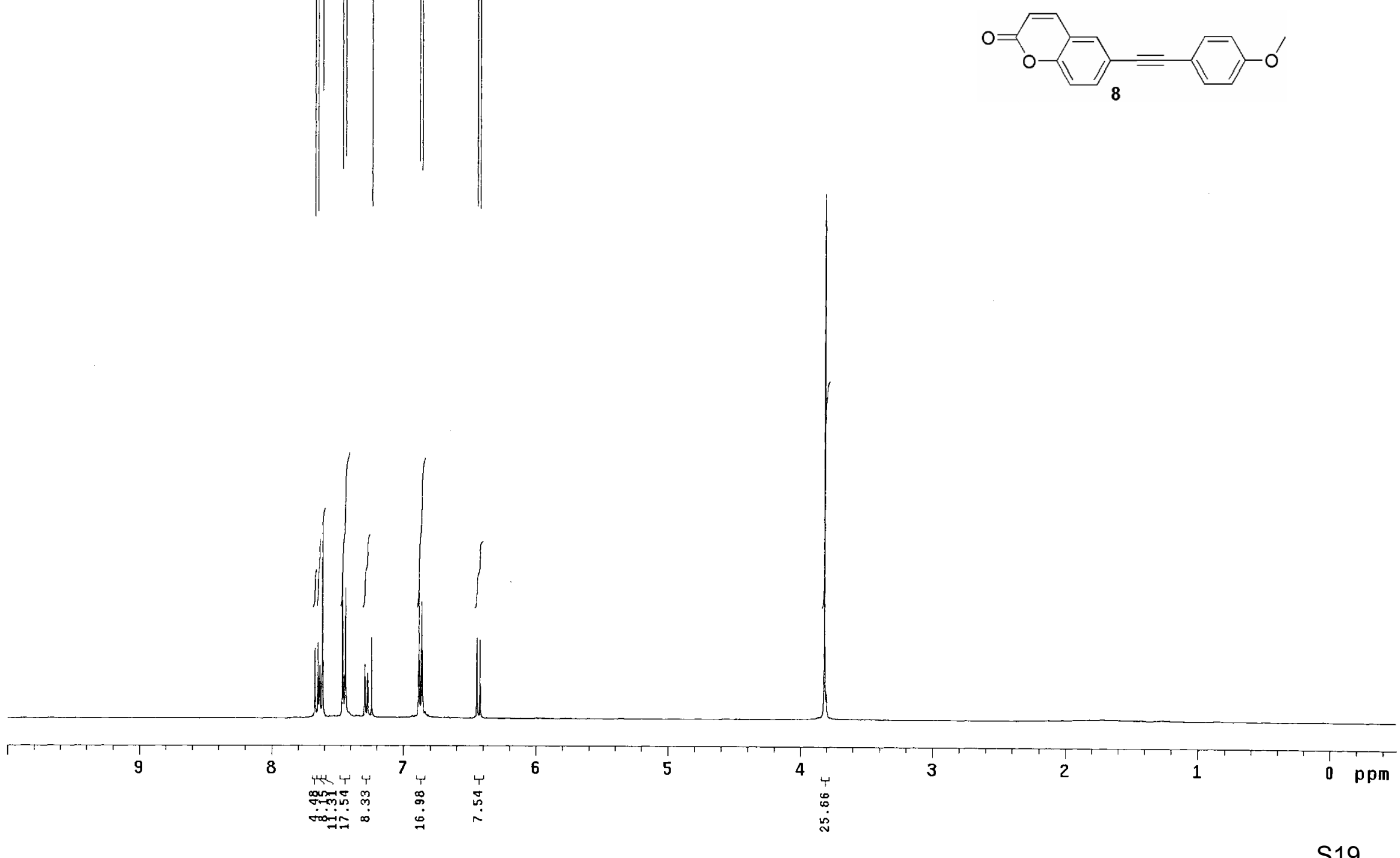


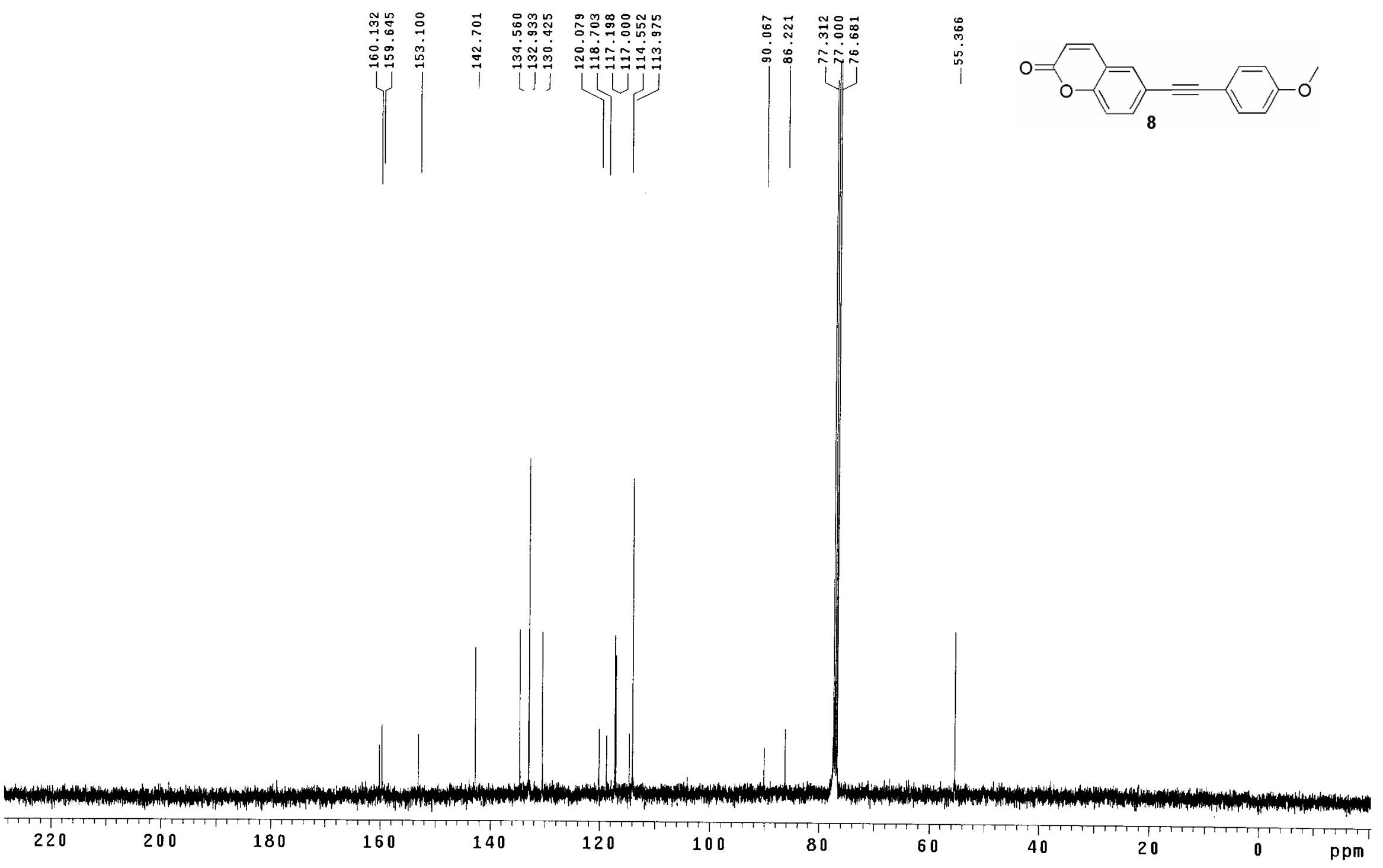


Pulse Sequence: s2pul
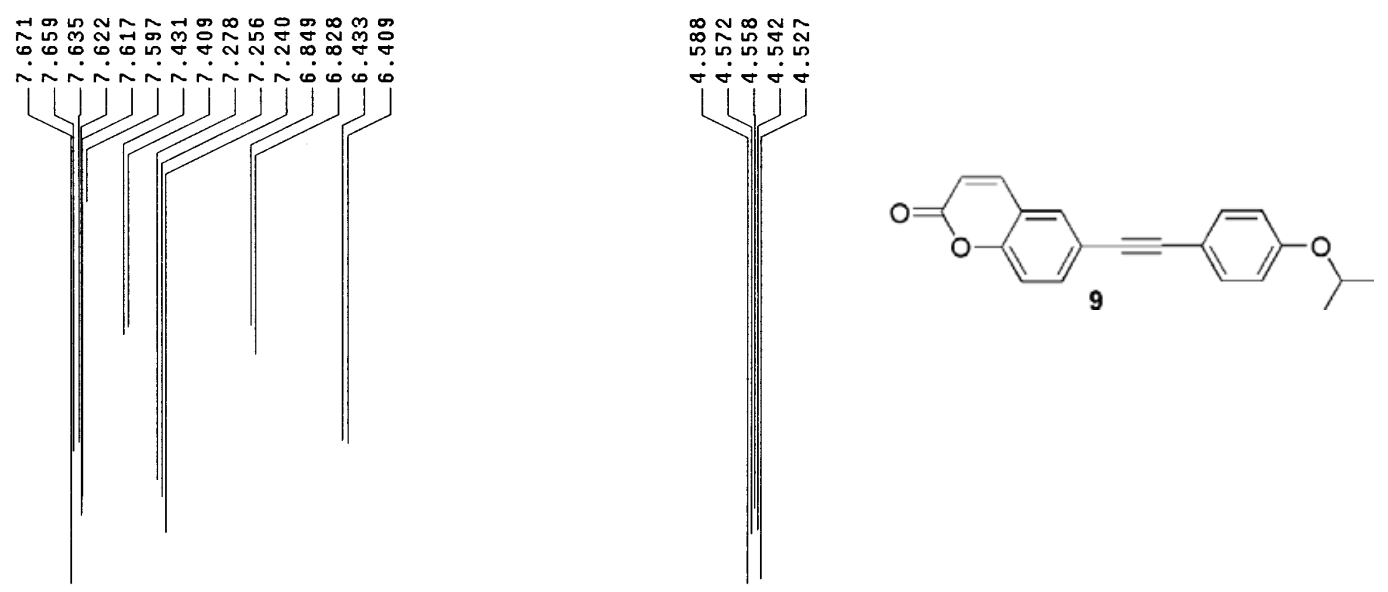

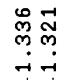

[j

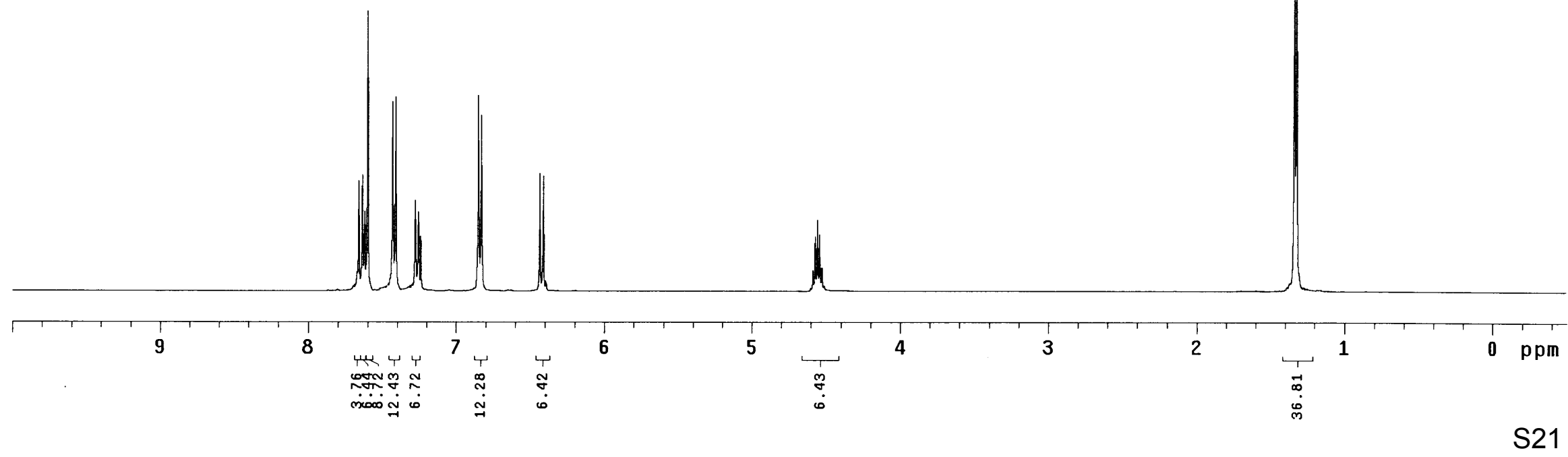



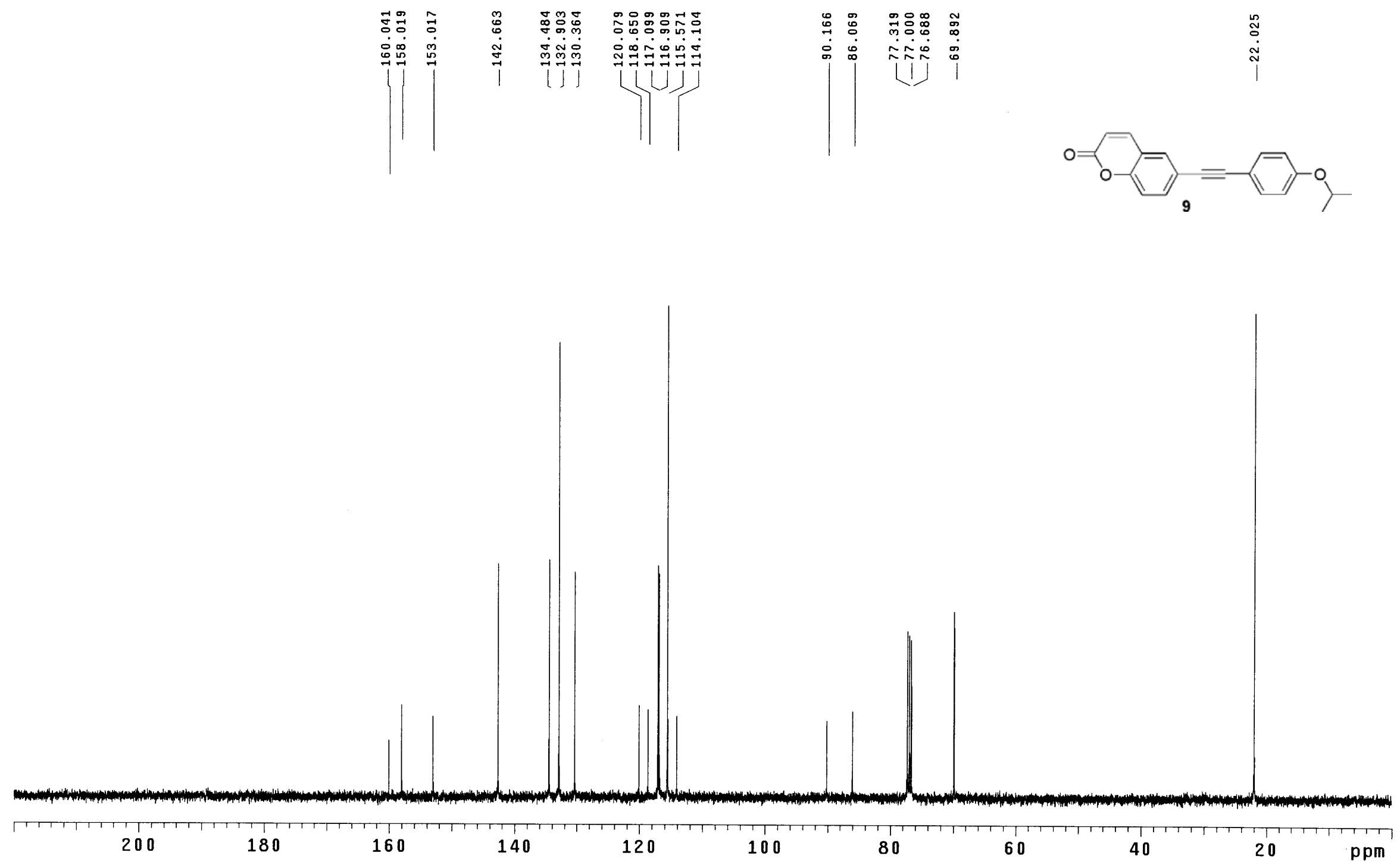
p2075

Pulse Sequence: s2pu1
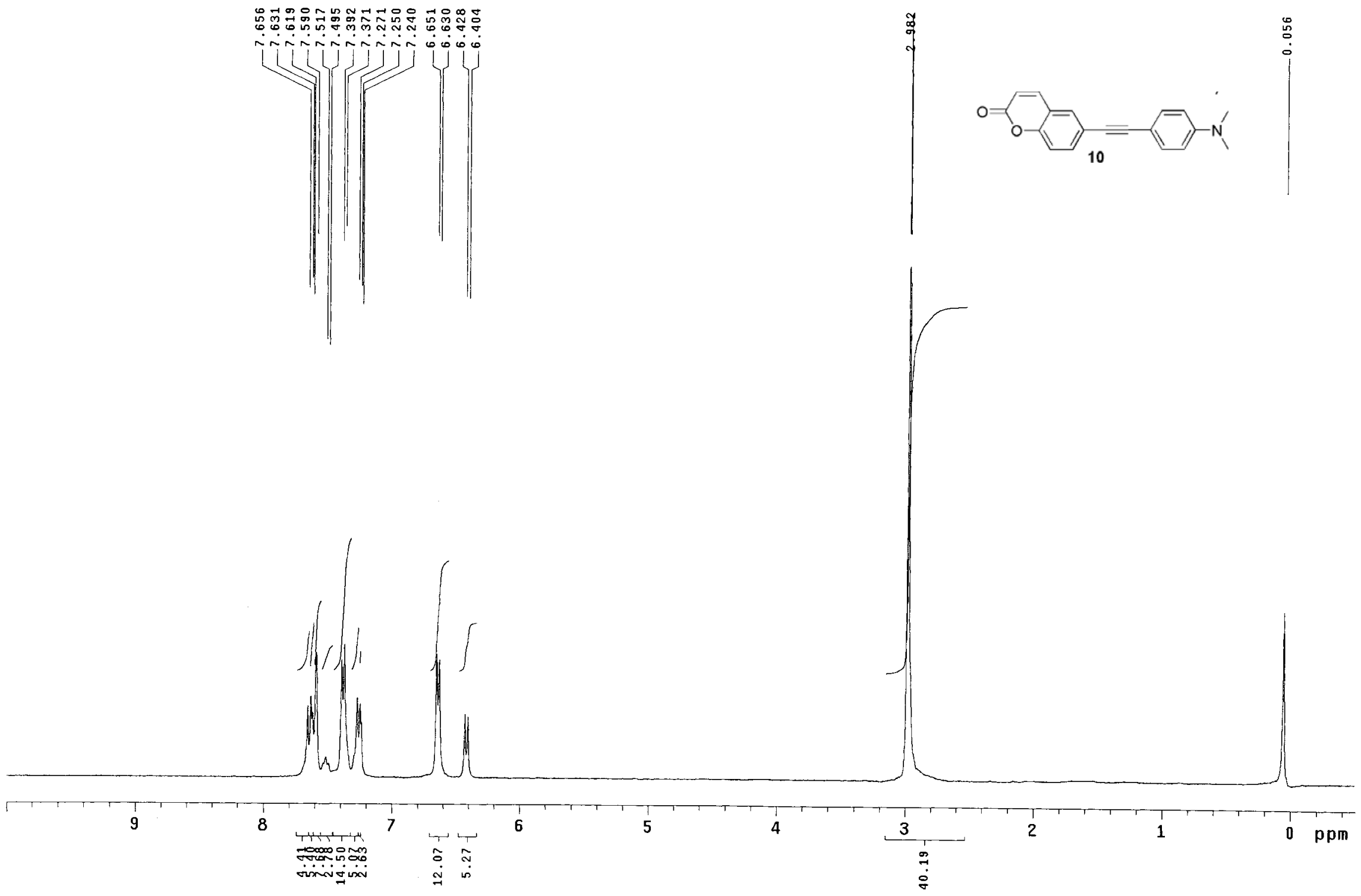

S23 
p2075-c13

Pulse Sequence: s2pu
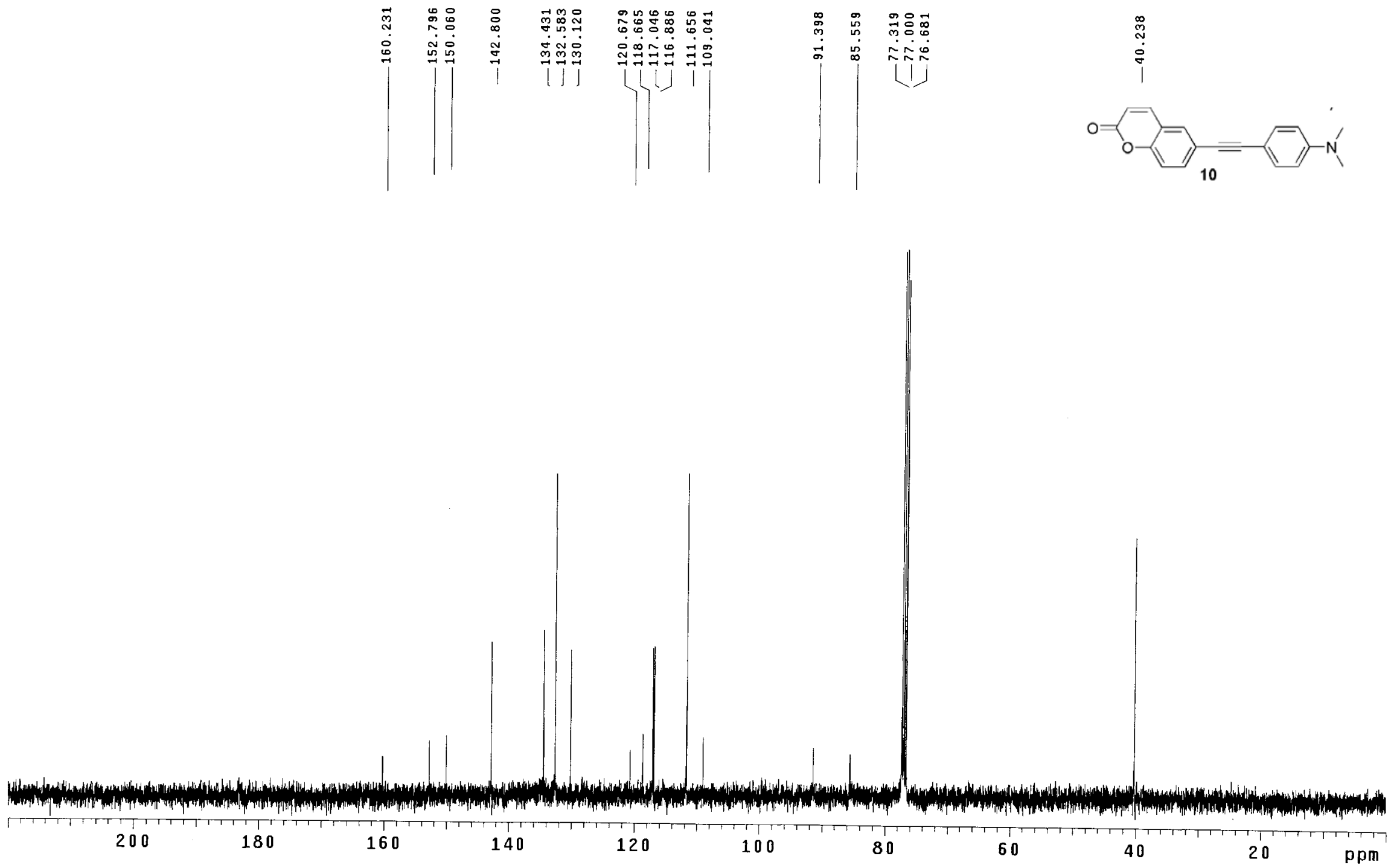


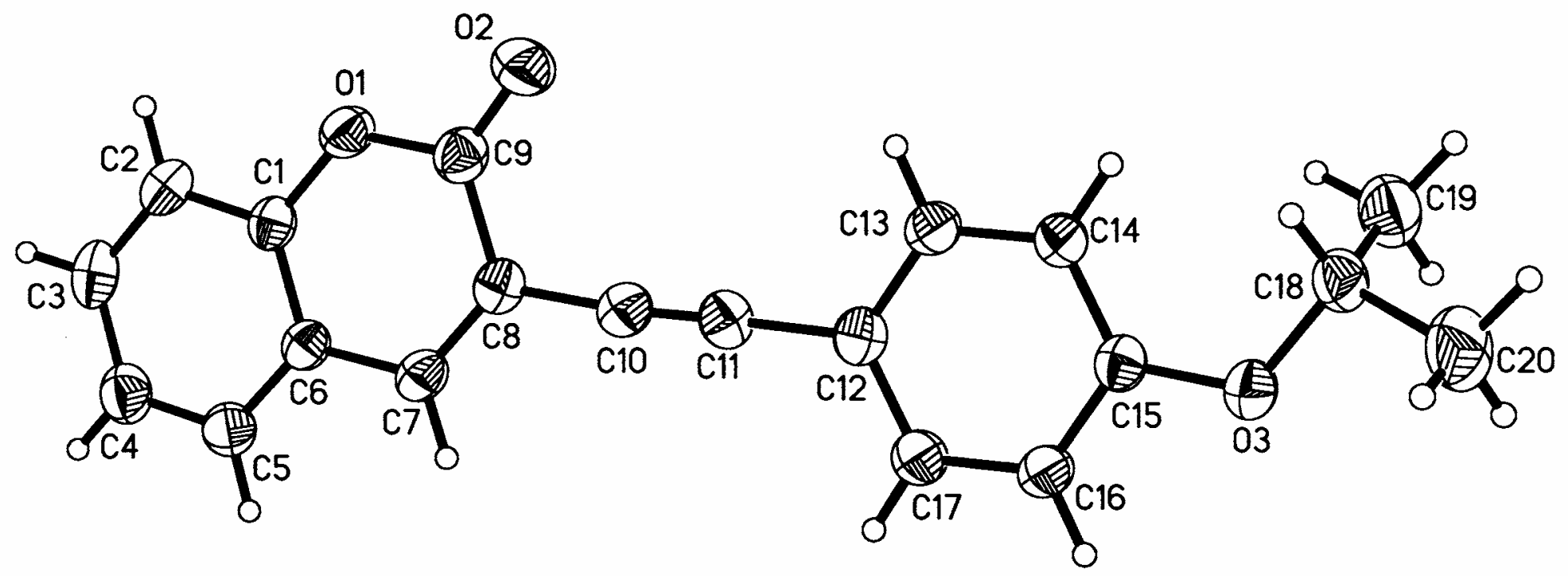

X-ray crystal structure (ORTEP) of compound 4 
Table 1. Crystal data and structure refinement for IC10547. (Compound 4 )

\begin{tabular}{|c|c|}
\hline Identification code & $i c 10547$ \\
\hline Diffractometer used & Nonius KappaCCD \\
\hline Empirical formula & $\mathrm{C}_{20} \mathrm{H}_{16} \mathrm{O}_{3}$ \\
\hline Formula weight & 304.33 \\
\hline Temperature & $295(2) \mathrm{K}$ \\
\hline Wavelength & $0.71073 \dot{A}$ \\
\hline Crystal system & Monoclinic \\
\hline Space group & $\mathrm{C} 2 / \mathrm{c}$ \\
\hline Unit cell dimensions & $\begin{array}{l}a=34.0680(4) \dot{A} \quad \text { alpha }=90^{\circ} \\
b=5.9080(2) \dot{A} \quad \text { beta }=115.704(2)^{\circ} \\
c=17.5600(7) \dot{A} \quad \text { gamma }=90^{\circ}\end{array}$ \\
\hline Volume, $\mathrm{z}$ & $3184.6(2) \dot{A}^{3}, 8$ \\
\hline Density (calculated) & $1.269 \mathrm{Mg} / \mathrm{m}^{3}$ \\
\hline Absorption coefficient & $0.085 \mathrm{~mm}^{-1}$ \\
\hline$F(000)$ & 1280 \\
\hline Crystal size & $0.30 \times 0.20 \times 0.15 \mathrm{~mm}$ \\
\hline$\theta$ range for data collection & 2.57 to $27.48^{\circ}$ \\
\hline Limiting indices & $-44 \leq h \leq 44,-6 \leq k \leq 7,-19 \leq I \leq 22$ \\
\hline Reflections collected & 8600 \\
\hline Independent reflections & $3576\left(\mathrm{R}_{\text {int }}=0.0434\right)$ \\
\hline Absorption correction & Multi-scan \\
\hline Max. and min. transmission & 0.988 and 0.914 \\
\hline Refinement method & Full-matrix least-squares on $F^{2}$ \\
\hline Data / restraints / parameters & $3523 / 0 / 209$ \\
\hline Goodness-of-fit on $F^{2}$ & 1.008 \\
\hline Final $R$ indices $[I>2 \sigma(I)]$ & $R 1=0.0582, w R 2=0.1354$ \\
\hline$R$ indices (all data) & $\mathrm{R} I=0.1377, \mathrm{wR2}=0.1734$ \\
\hline Extinction coefficient & $0.0045(12)$ \\
\hline Largest diff. peak and hole & 0.258 and $-0.165 \mathrm{e}^{-3}$ \\
\hline
\end{tabular}


Table 2. Bond lengths $[\AA]$ and angles $\left[{ }^{\circ}\right]$ for 10547. (Compound 4)

\begin{tabular}{|c|c|c|c|}
\hline$O(1)-C(9)$ & $1.371(2)$ & $O(1)-C(1)$ & $1.382(2)$ \\
\hline$O(2)-C(9)$ & $1.201(3)$ & $O(3)-C(15)$ & $1.357(2)$ \\
\hline$O(3)-C(18)$ & $1.445(3)$ & $C(1)-C(2)$ & $1.378(3)$ \\
\hline$C(1)-C(6)$ & $1.389(3)$ & $C(2)-C(3)$ & $1.381(3)$ \\
\hline$C(3)-C(4)$ & $1.384(3)$ & $C(4)-C(5)$ & $1.369(3)$ \\
\hline$C(5)-C(6)$ & $1.393(3)$ & $C(6)-C(7)$ & $1.428(3)$ \\
\hline$C(7)-C(8)$ & $1.354(3)$ & $C(8)-C(10)$ & $1.463(3)$ \\
\hline$C(8)-C(9)$ & $1.466(3)$ & $C(10)-C(11)$ & $1.125(3)$ \\
\hline$C(11)-C(12)$ & $1.473(3)$ & $C(12)-C(13)$ & $1.391(3)$ \\
\hline$C(12)-C(17)$ & $1.400(3)$ & $C(13)-C(14)$ & $1.371(3)$ \\
\hline$C(14)-C(15)$ & $1.383(3)$ & $C(15)-C(16)$ & $1.386(3)$ \\
\hline$C(16)-C(17)$ & $1.362(3)$ & $C(18)-C(19)$ & $1.492(3)$ \\
\hline$C(18)-C(20)$ & $1.503(3)$ & & \\
\hline$C(9)-O(1)-C(1)$ & $122.7(2)$ & $C(15)-O(3)-C(18)$ & $120.1(2)$ \\
\hline$C(2)-C(1)-O(1)$ & $116.8(2)$ & $C(2)-C(1)-C(6)$ & $122.5(2)$ \\
\hline$O(1)-C(1)-C(6)$ & $120.6(2)$ & $C(1)-C(2)-C(3)$ & $117.9(2)$ \\
\hline$C(2)-C(3)-C(4)$ & $121.2(2)$ & $C(5)-C(4)-C(3)$ & $119.6(2)$ \\
\hline$C(4)-C(5)-C(6)$ & $121.0(2)$ & $C(1)-C(6)-C(5)$ & $117.6(2)$ \\
\hline$C(1)-C(6)-C(7)$ & $118.2(2)$ & $C(5)-C(6)-C(7)$ & $124.1(2)$ \\
\hline$C(8)-C(7)-C(6)$ & $121.2(2)$ & $C(7)-C(8)-C(10)$ & $124.3(2)$ \\
\hline$C(7)-C(8)-C(9)$ & $120.3(2)$ & $C(10)-C(8)-C(9)$ & $115.5(2)$ \\
\hline$O(2)-C(9)-O(1)$ & $117.5(2)$ & $O(2)-C(9)-C(8)$ & $125.6(2)$ \\
\hline$O(1)-C(9)-C(8)$ & $116.9(2)$ & $C(11)-C(10)-C(8)$ & $174.9(2)$ \\
\hline$C(10)-C(11)-C(12)$ & $178.3(2)$ & $C(13)-C(12)-C(17)$ & $117.8(2)$ \\
\hline$C(13)-C(12)-C(11)$ & $119.5(2)$ & $C(17)-C(12)-C(11)$ & $122.6(2)$ \\
\hline$C(14)-C(13)-C(12)$ & $121.1(2)$ & $C(13)-C(14)-C(15)$ & $120.5(2)$ \\
\hline$O(3)-C(15)-C(14)$ & $125.8(2)$ & $O(3)-C(15)-C(16)$ & $115.6(2)$ \\
\hline$C(14)-C(15)-C(16)$ & $118.6(2)$ & $C(17)-C(16)-C(15)$ & $121.2(2)$ \\
\hline$C(16)-C(17)-C(12)$ & $120.7(2)$ & $O(3)-C(18)-C(19)$ & $110.8(2)$ \\
\hline$O(3)-C(18)-C(20)$ & $104.8(2)$ & $C(19)-C(18)-C(20)$ & $112.4(2)$ \\
\hline
\end{tabular}




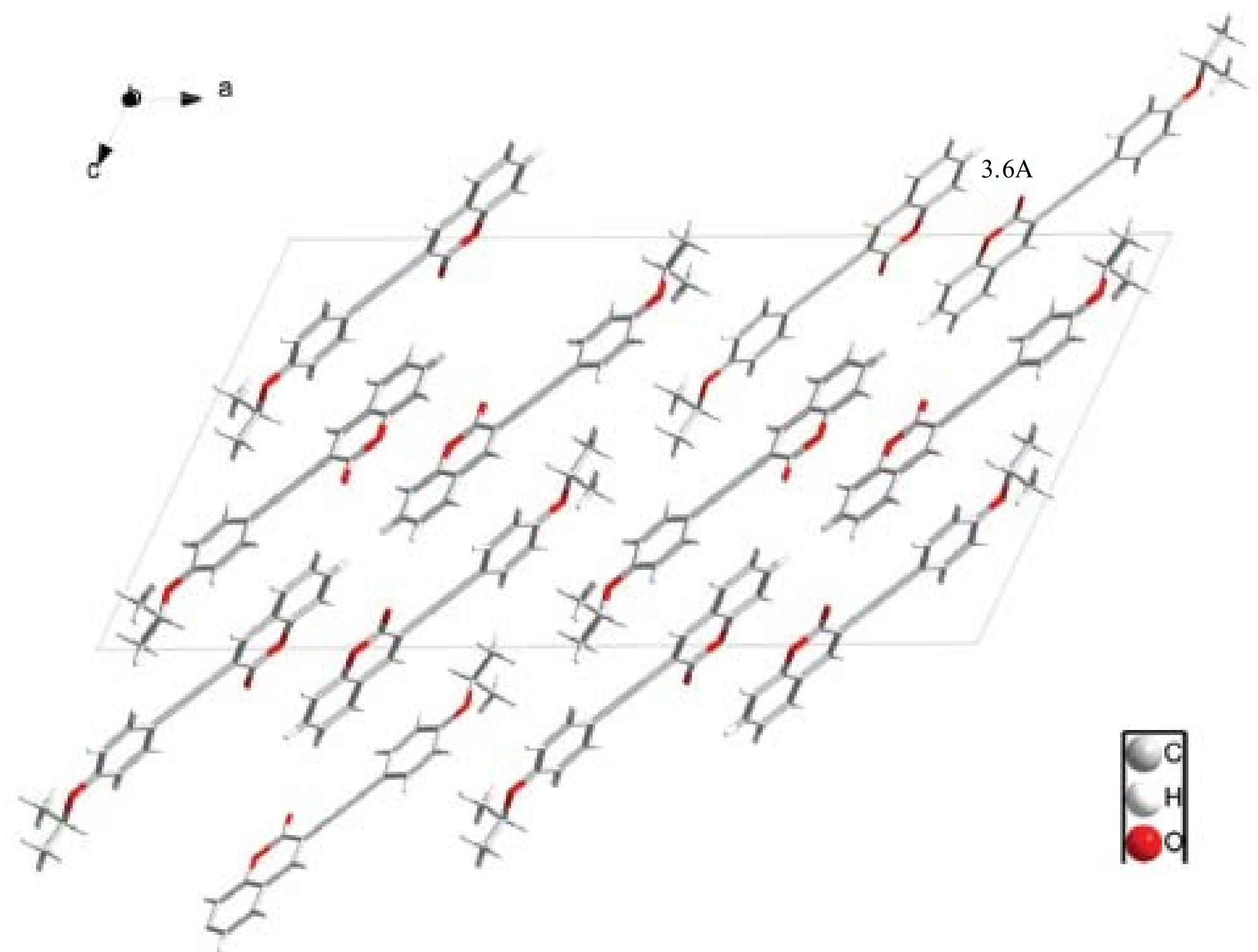

Crystal packing diagram showing $\pi$-interaction in $\mathbf{4}$ 

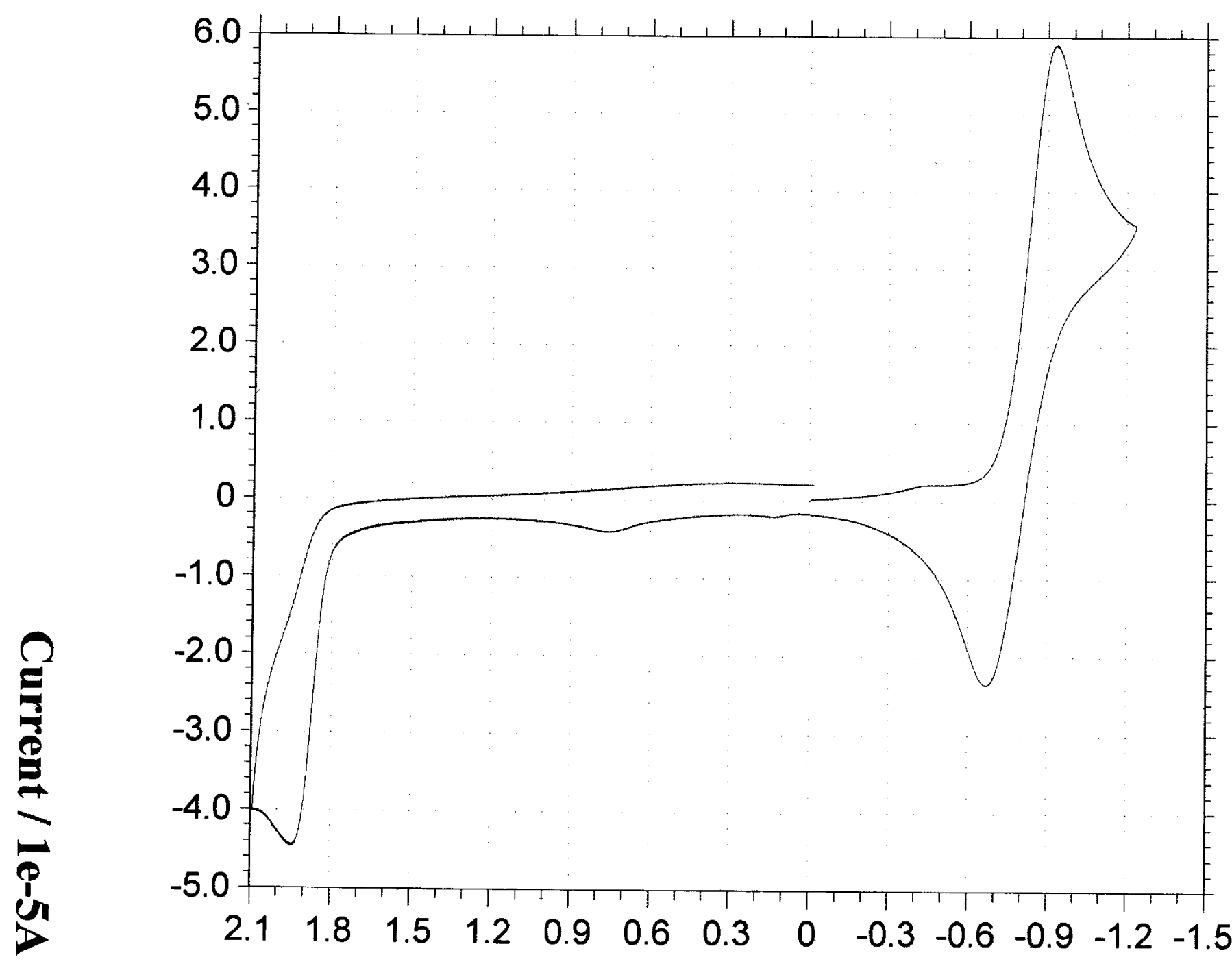

Mar. 18, 2004

$14: 14: 02$

Tech: CV

File: 3-H-2.bin

Init $E(V)=0$

High $E(V)=2.5$

Low $E(V)=-2$

Init $P / N=N$

Scan Rate $(\mathrm{V} / \mathrm{s})=0.05$

Segment $=3$

Smpl Interval $(V)=0.001$

Quiet Time $(s)=2$

Sensitivity $(A N)=1 e-5$

CV curve of 1

\section{Potential / V}



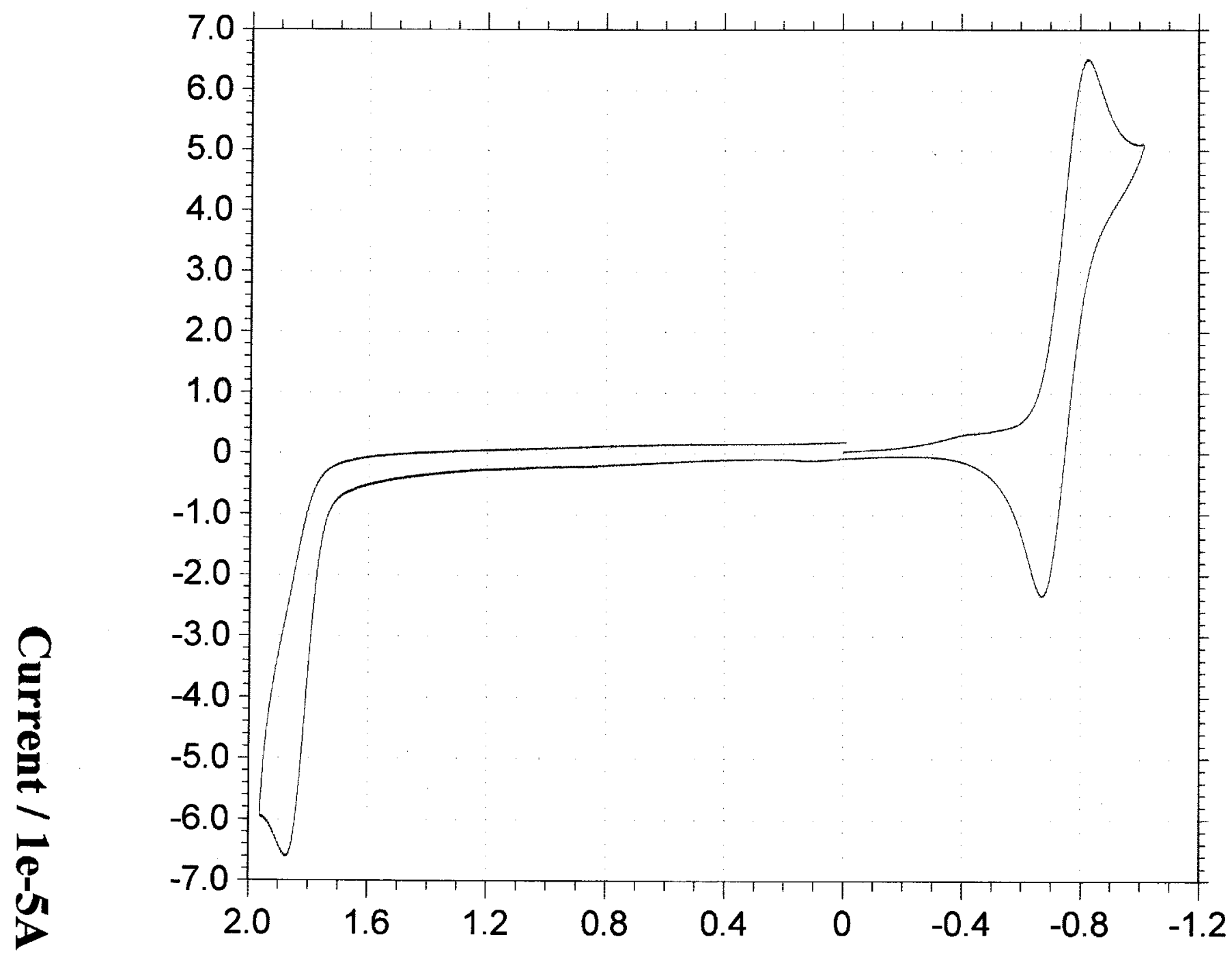

Feb. 20, 2004 12:55:30

Tech: CV

File: 3-CH3.bin

Init $E(V)=0$

High $E(V)=2$

Low $E(V)=-2$

Init $P / N=N$

Scan Rate $(\mathrm{V} / \mathrm{s})=0.05$

Segment $=3$

Smpl Interval $(V)=0.001$

Quiet Time (s) $=2$

Sensitivity $(A N)=1 e-5$

2nd $E(V)=0$

Sens $2(A N)=1 e-6$

CV curve of 2

\section{Potential / V}



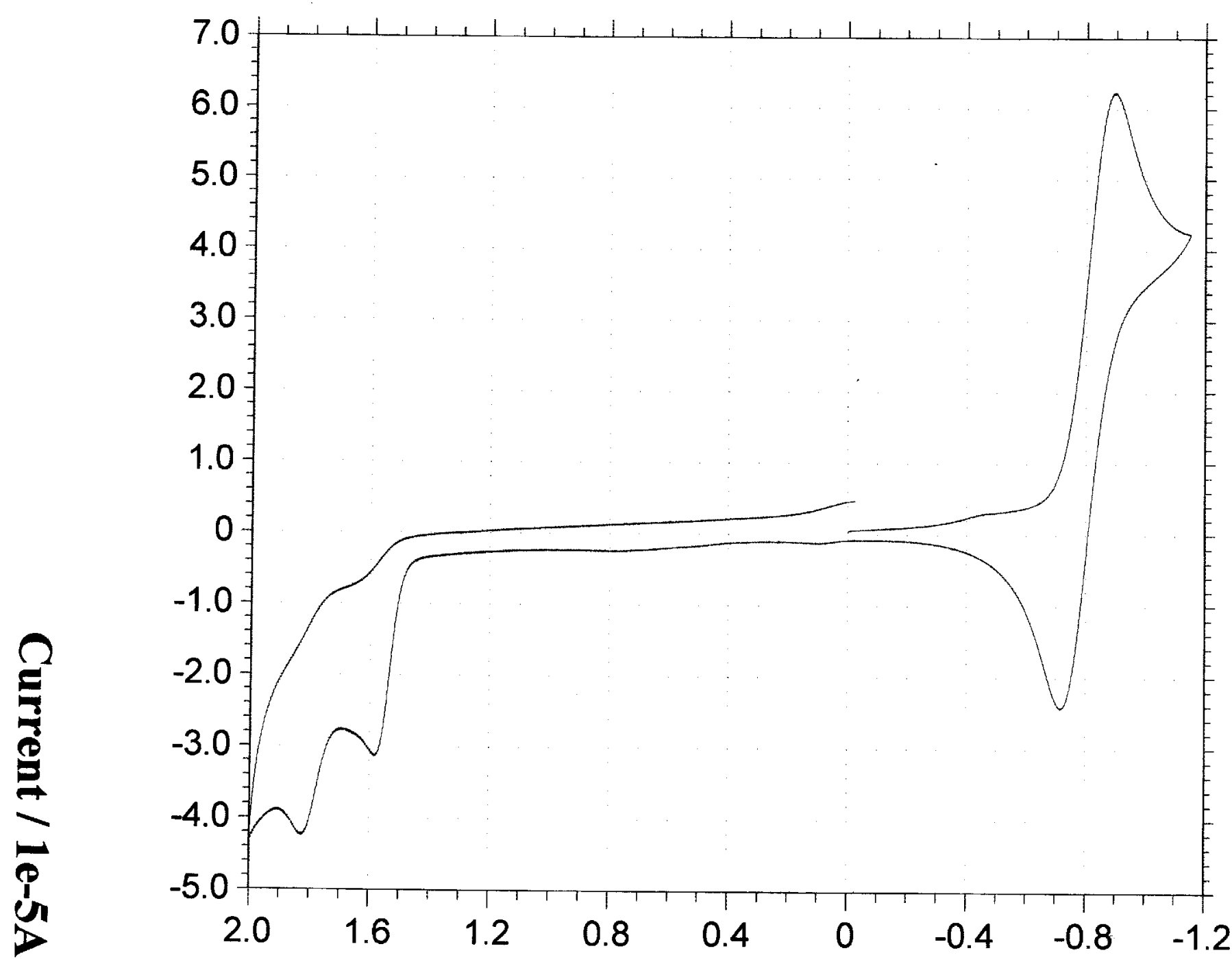

Mar. 18, 2004 15:58:56 Tech: CV

File: 3-OCH3.bin

Init $E(V)=0$

High $E(V)=2$

Low $E(V)=-3$

Init $P / N=N$

Scan Rate $(\mathrm{V} / \mathrm{s})=0.05$

Segment $=3$

Smpl Interval $(V)=0.001$

Quiet Time $(s)=2$

Sensitivity $(A N)=1 e-5$

CV curve of 3

\section{Potential / V}




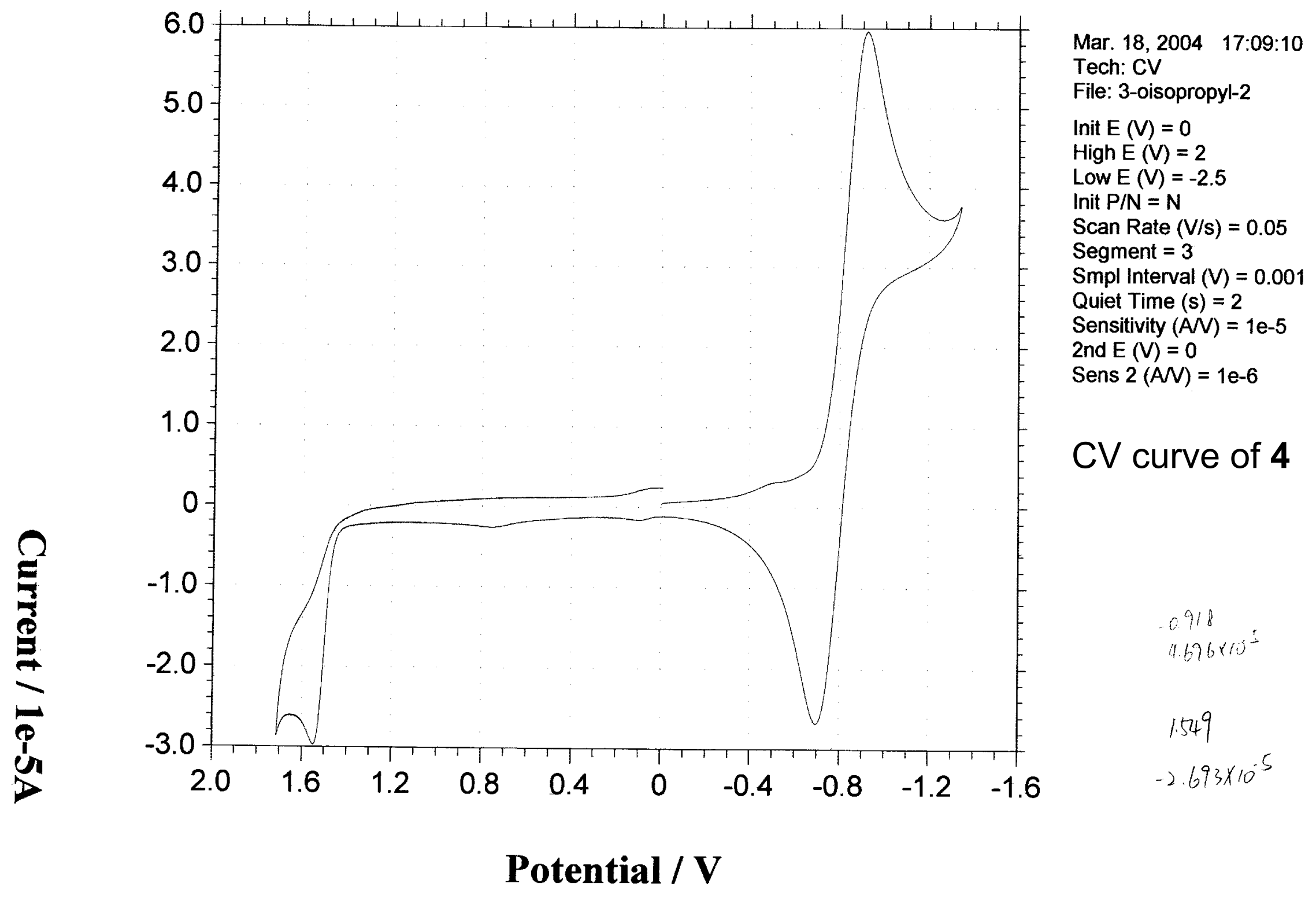



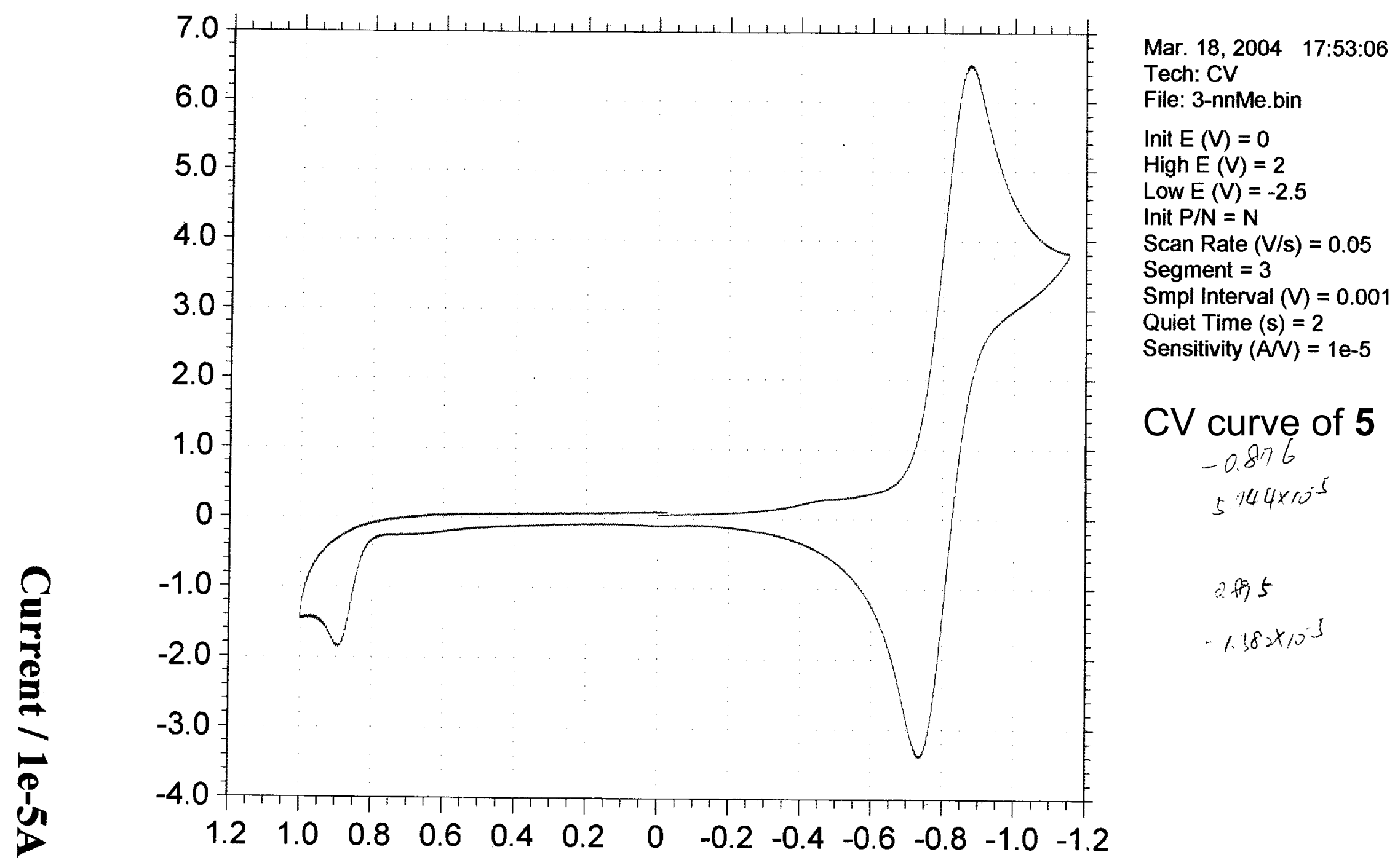

\section{Potential / V}




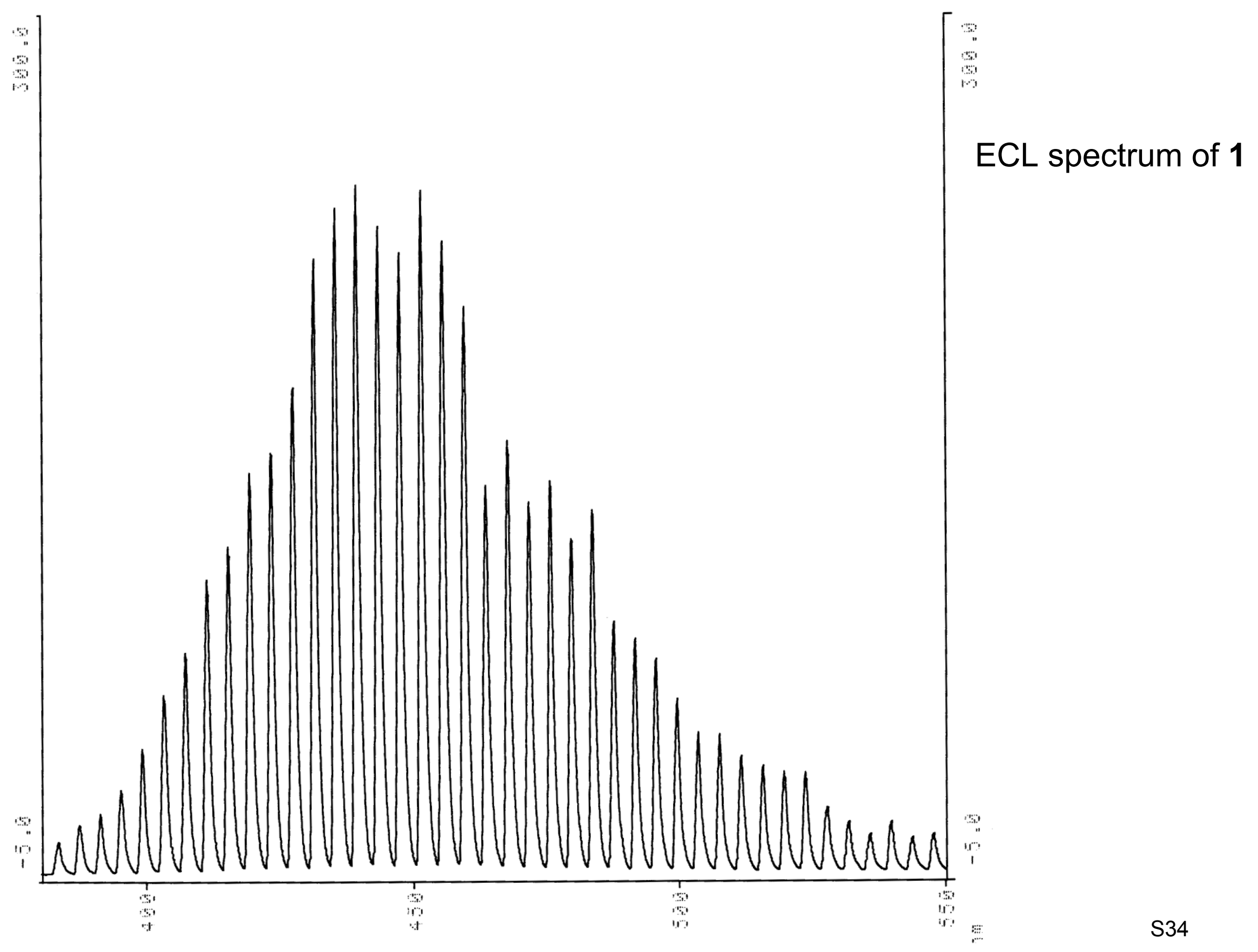




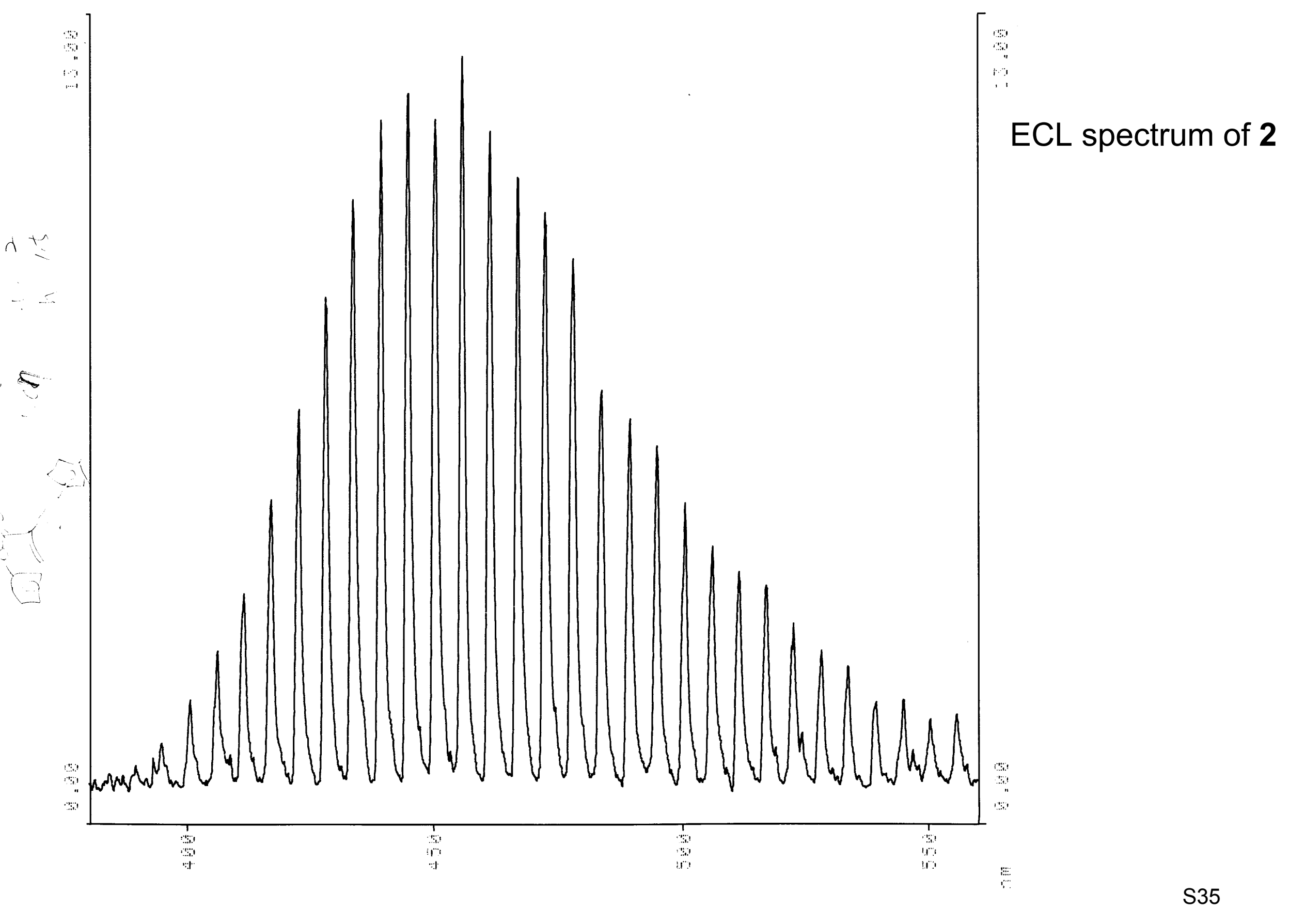




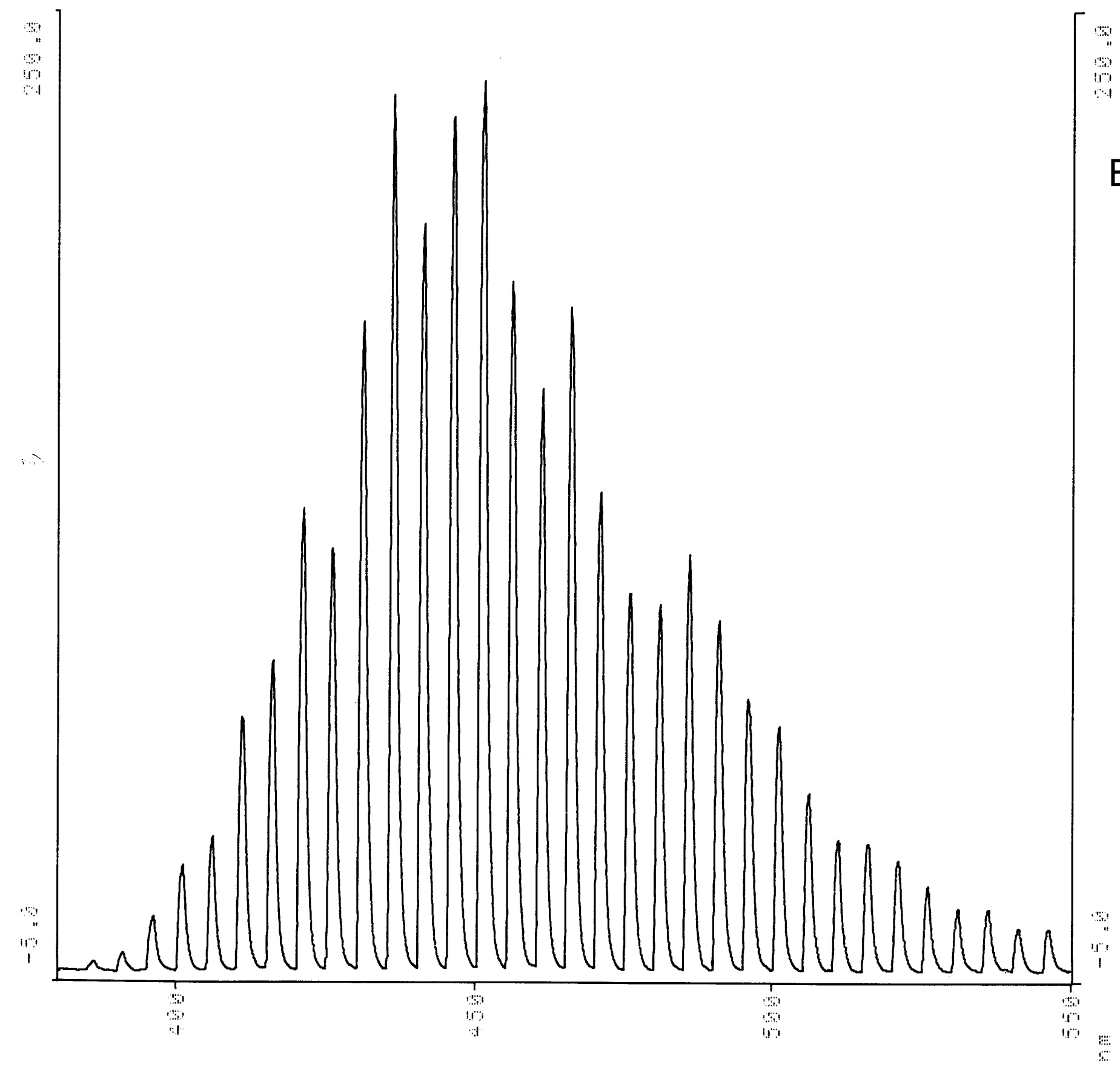

ECL spectrum of 4 


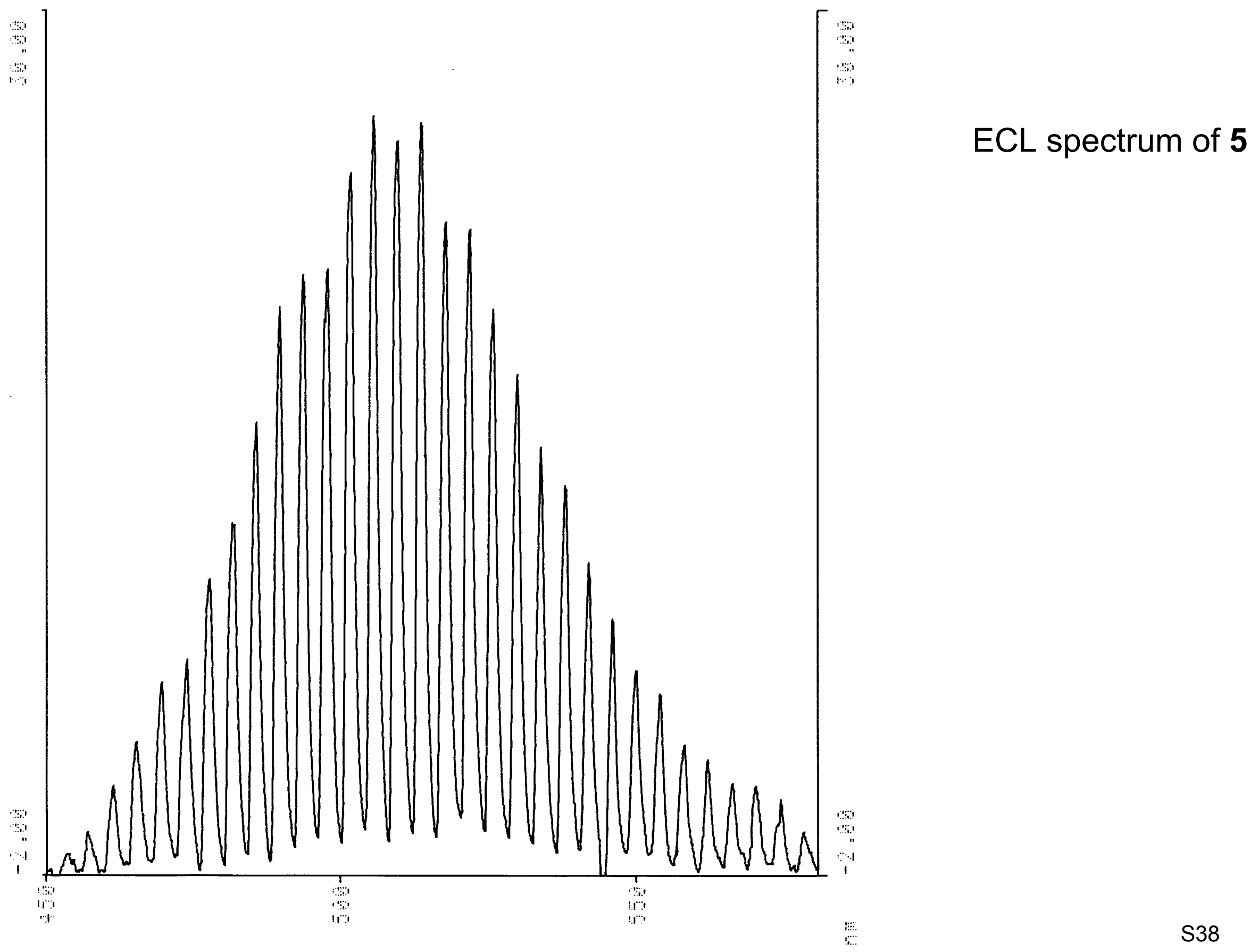




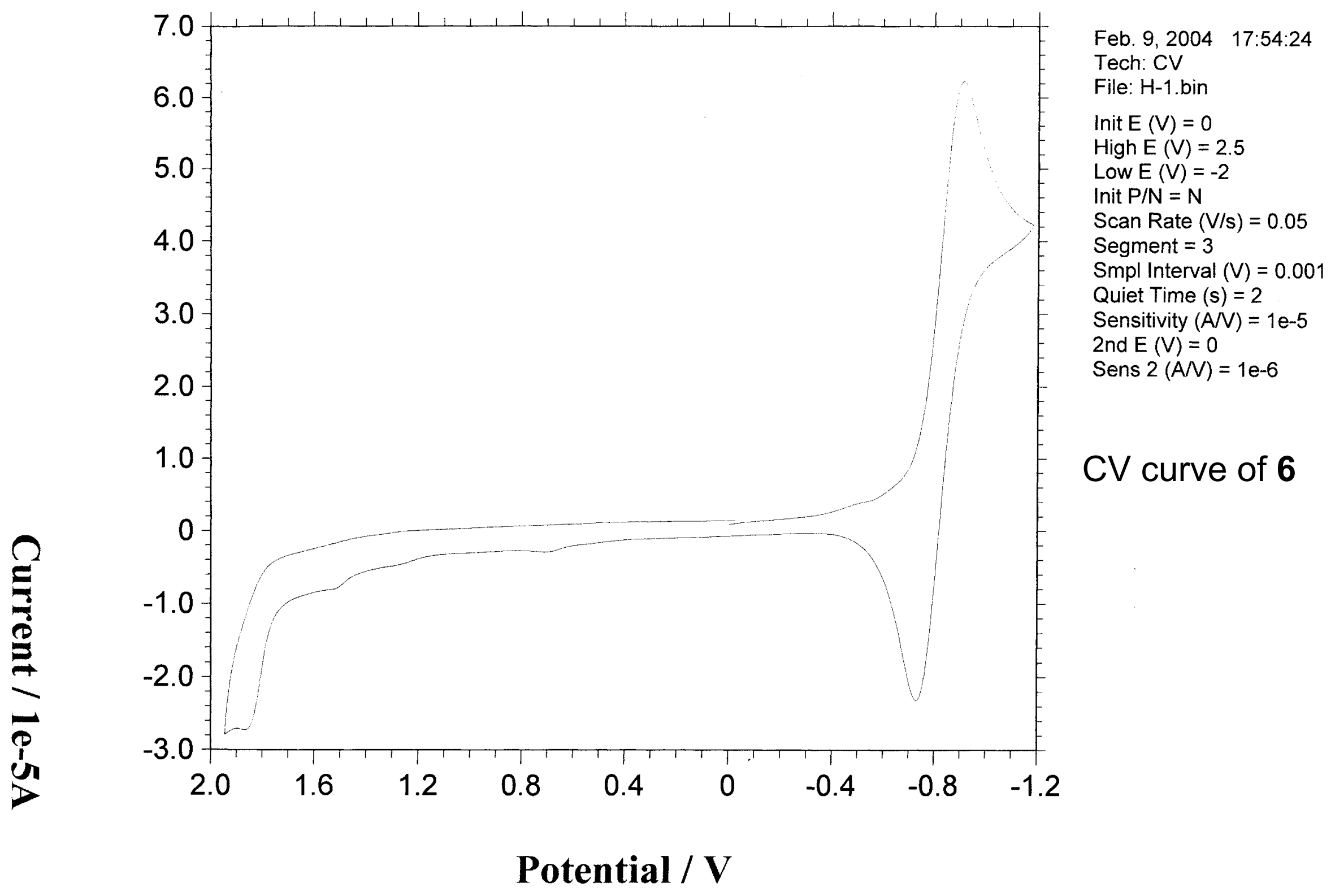



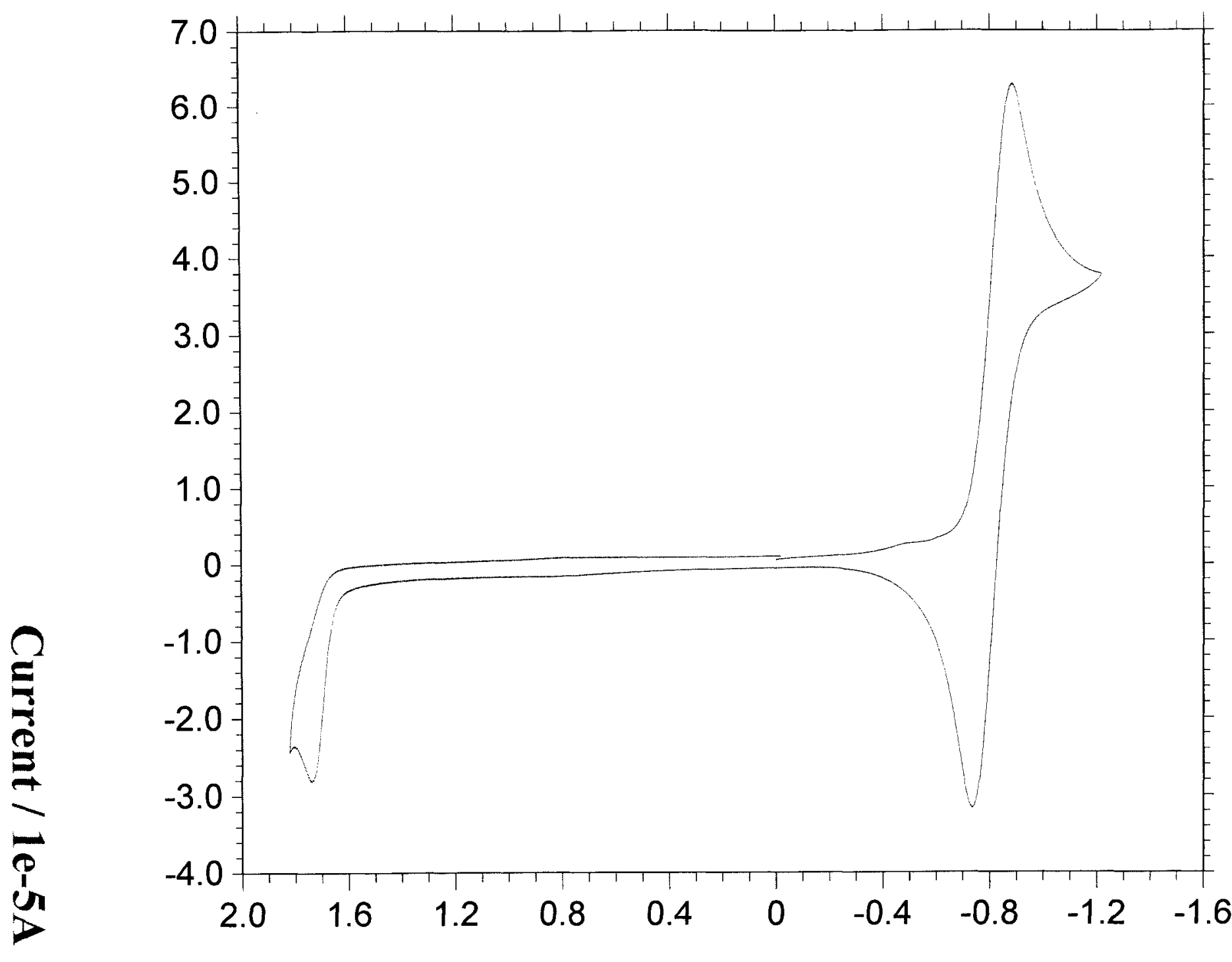

Feb. 9, 2004

$18: 22: 59$

Tech: CV

File: ch3

Init $E(V)=0$

High $E(V)=2.5$

Low $E(V)=-2$

Init $\mathrm{P} / \mathrm{N}=\mathrm{N}$

Scan Rate $(\mathrm{V} / \mathrm{s})=0.05$

Segment $=3$

Smpl Interval $(\mathrm{V})=0.001$

Quiet Time (s) $=2$

Sensitivity $(A N)=1 e-5$

2nd $E(V)=0$

Sens $2(A N)=1 e-6$

CV curve of 7

\section{Potential / V}



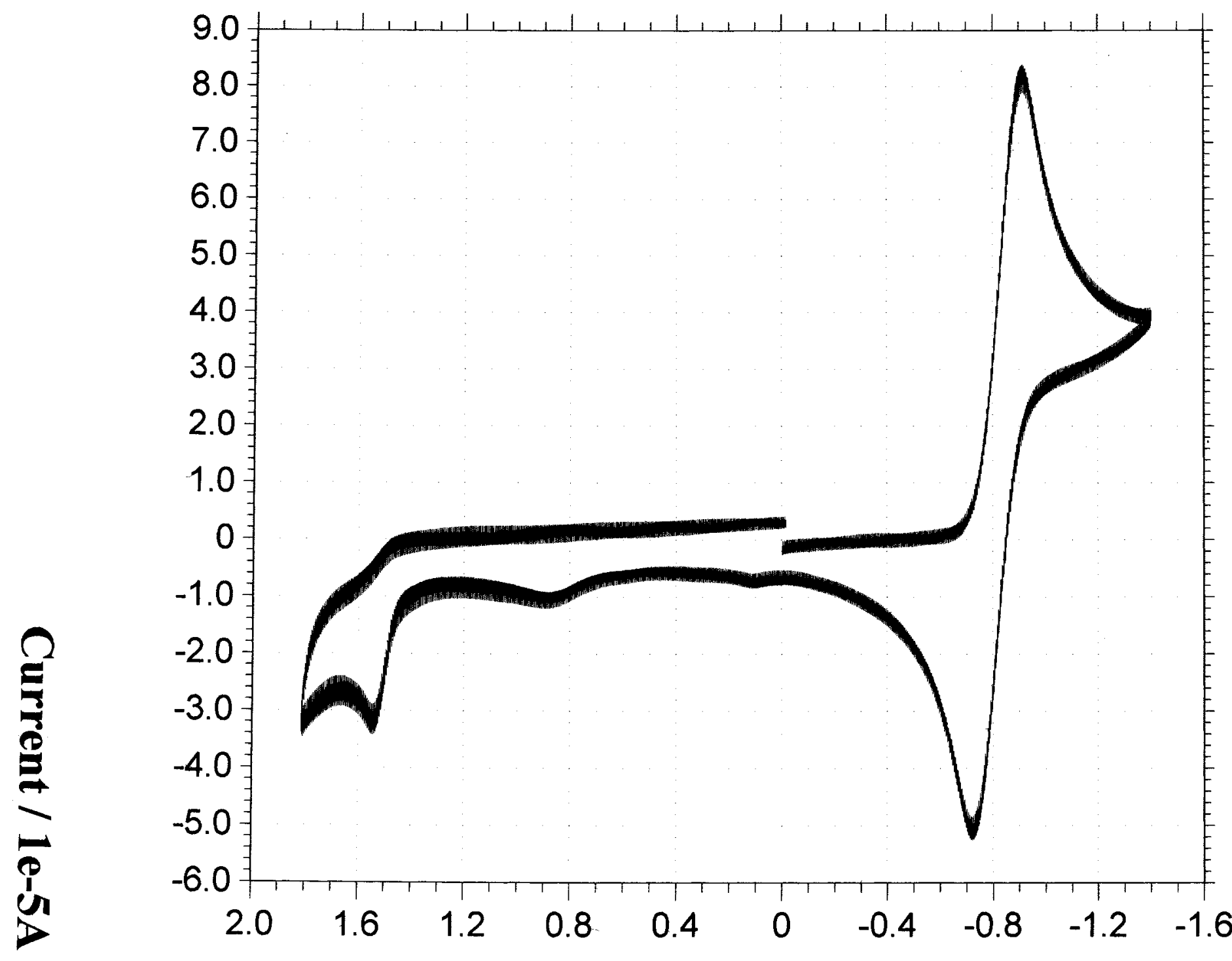

Dec. 26, 2003 14:23:47 Tech: CV

File: ome.bin

Init $E(V)=0$

High $E(V)=2$

Low $E(V)=-2$

Init $\mathrm{P} / \mathrm{N}=\mathrm{N}$

Scan Rate $(\mathrm{V} / \mathrm{s})=0.1$

Segment $=3$

Smpl Interval $(V)=0.001$

Quiet Time $(\mathrm{s})=2$

Sensitivity $(A N)=1 e-5$

CV curve of 8

\section{Potential / V}




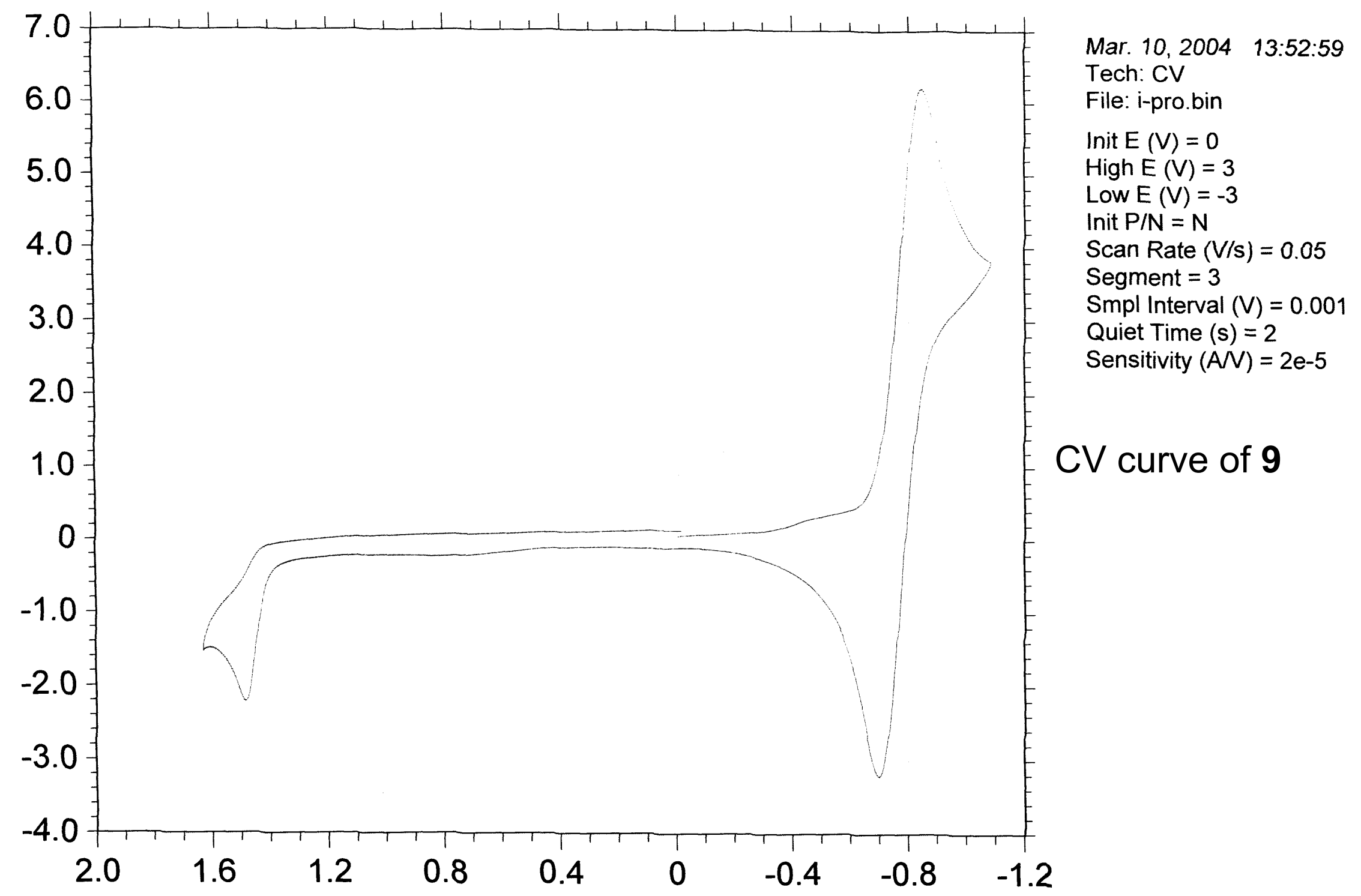




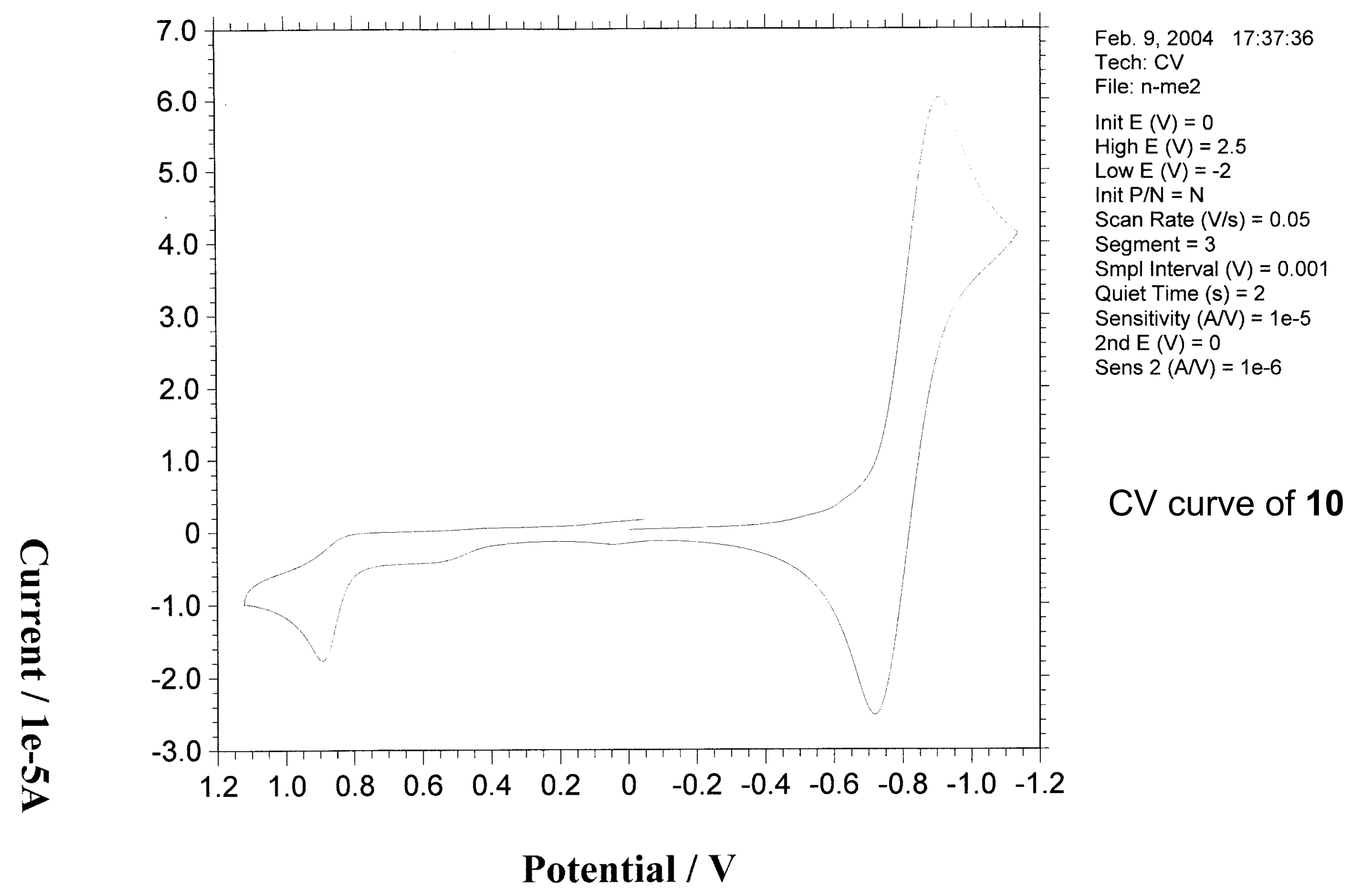




\section{ECL spectrum of 6}

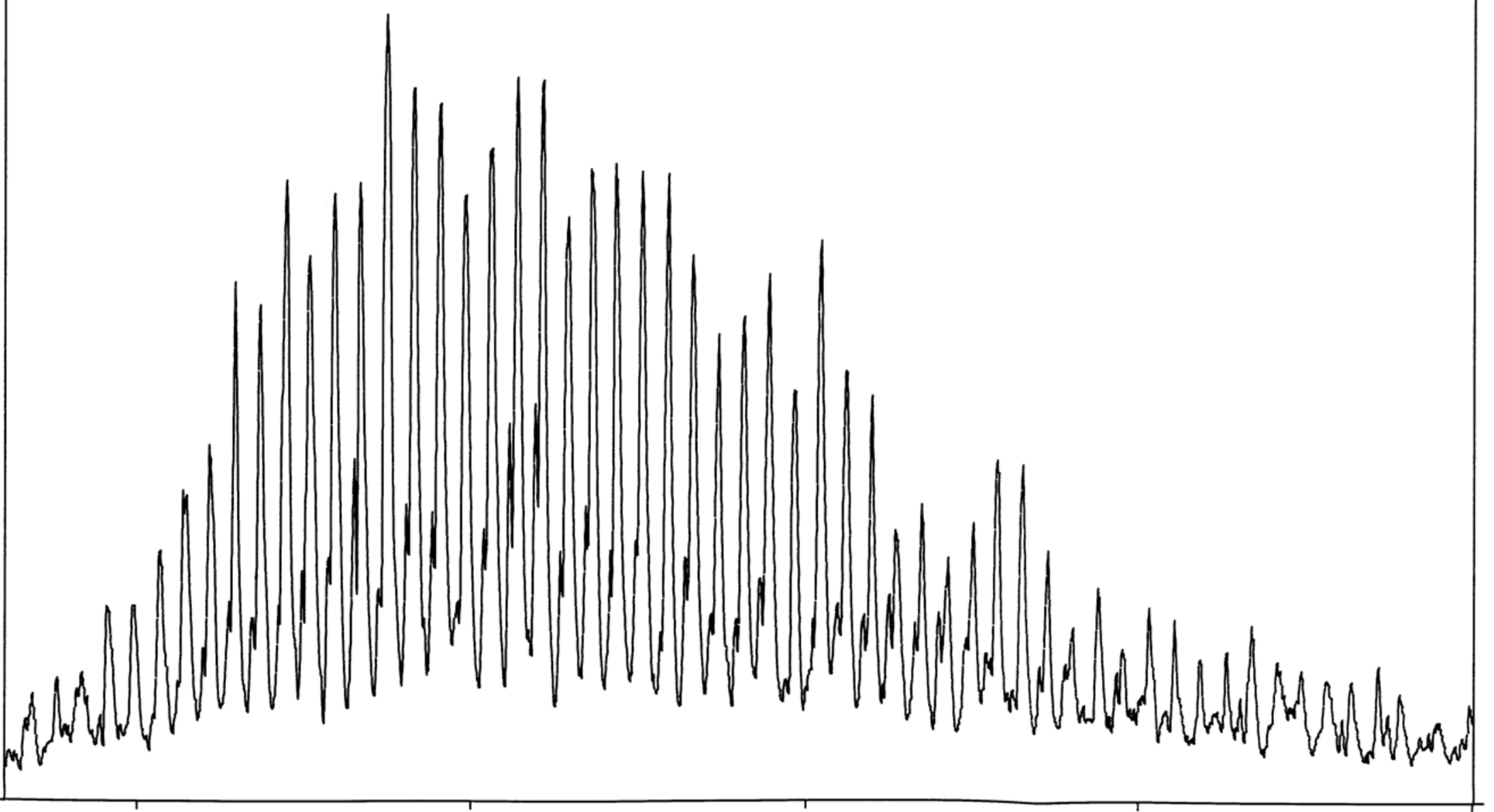




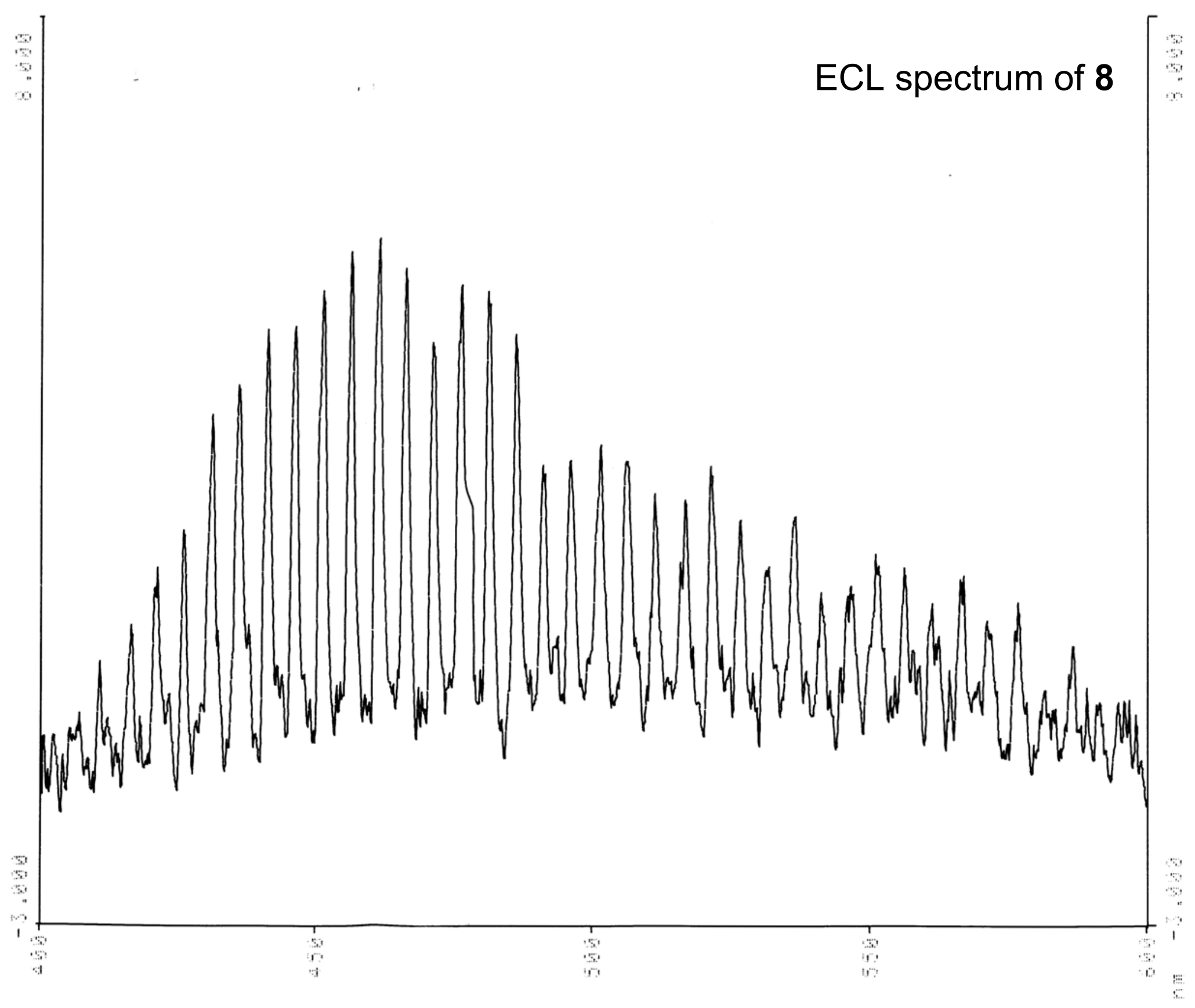




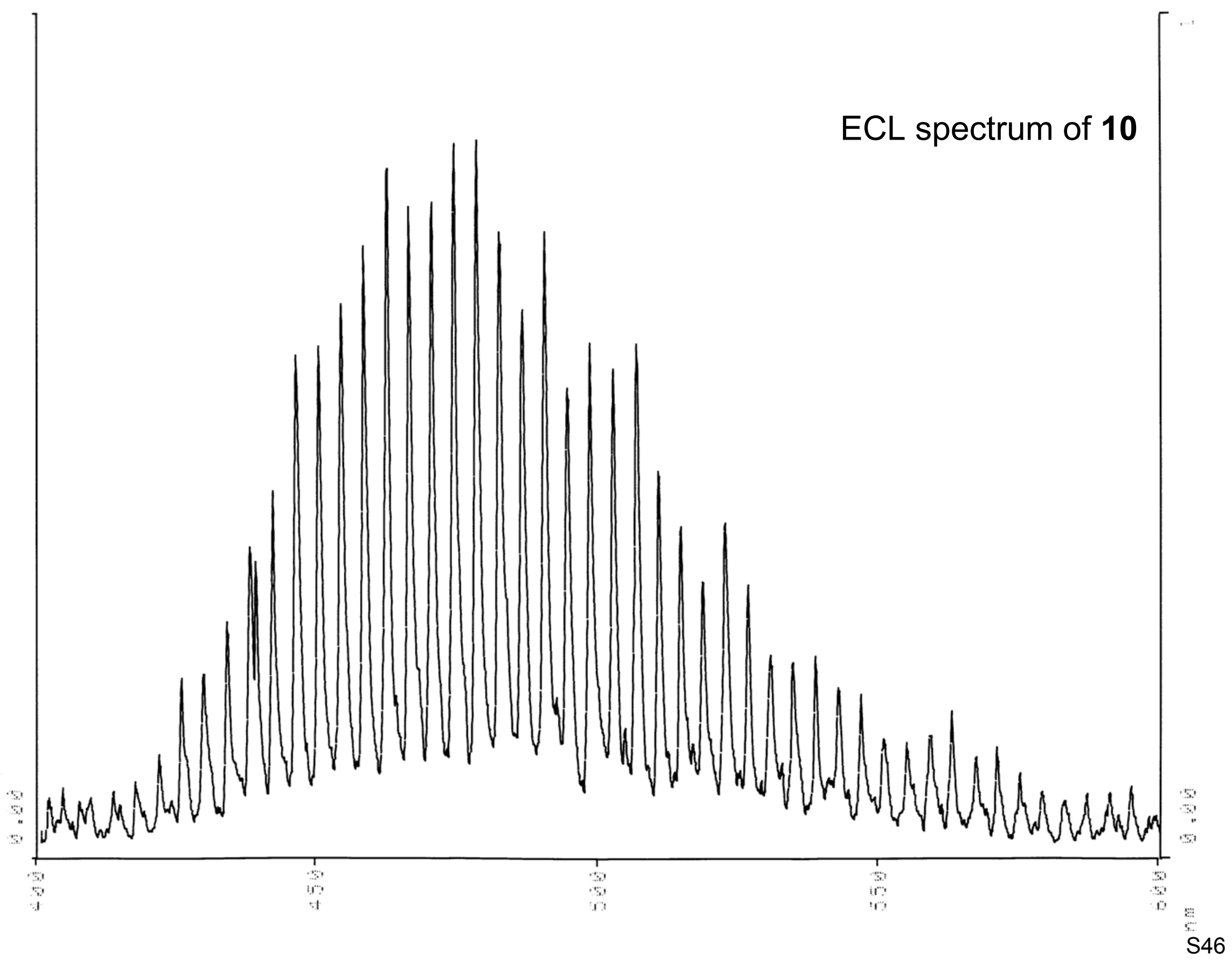




\section{Procedure used for performing theoretical calculations}

The program used:

Level of theory:

Basis set:

\section{Computational method:}

Structures were drawn at the Entry-level of input and minimized. Calculations were set up to perform geometry optimization in order to obtain equilibrium geometry for the molecule at the ground state with Semi-empirical method PM3 from its initial geometry subject to symmetry. The total charge was neutral and multiplicity was Singlet.

Print was requested for atomic charges, thermodynamics, vibrational modes, orbitals and energies. The required surfaces were selected and the problem was submitted. When the calculations were completed, the orbital surfaces were mapped and other properties were outputted.

The following pages contain the minimum energy structures of 1, 6, $\mathbf{5}$ and $\mathbf{1 0}$ as appropriate and their energies and Cartesian coordinates. No imaginary frequencies were present.

\author{
Spartan'04 (for Windows) \\ Semi-empirical Parameterization Method 3 (also called Parameter Model 3, PM3) \\ Predefined basis sets as provided by Spartan'04 \\ (restricted to minimal valence-only representation; \\ incorporates restricted Hartree-Fock model)
}




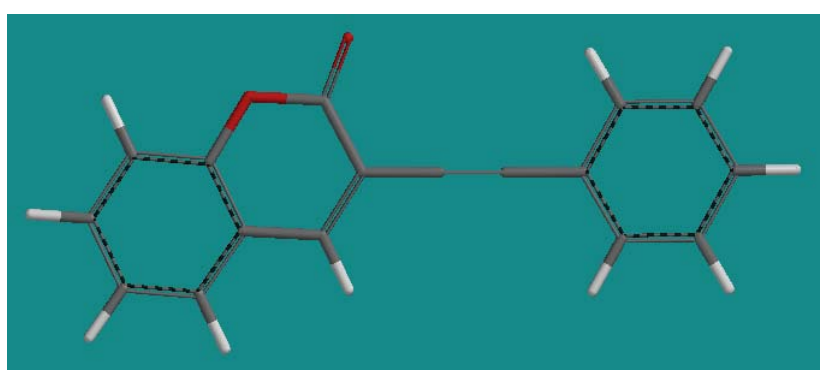

Optimized ground state structure of 1

Energy: $38.11 \mathrm{kcal} / \mathrm{mol}$

\begin{tabular}{rrrrr}
\multicolumn{5}{c}{ Cartesian Coordinates (Angstroms) } \\
Atom & X & Y & Z \\
1 H H1 & $-1-1.0388082$ & 0.0000000 & -5.2892747 \\
2 C C1 & -1.0151203 & 0.0000000 & -4.8969663 \\
3 C C4 & 1.6031850 & 0.0000000 & -3.8796035 \\
4 C C2 & -0.7866845 & 0.0000000 & -3.5110668 \\
5 C C6 & 0.0749204 & 0.0000000 & -5.7503883 \\
6 C C5 & 1.3794273 & 0.0000000 & -5.2448048 \\
7 C C3 & 0.5186479 & 0.0000000 & -2.9898926 \\
8 H H6 & -0.0844317 & 0.0000000 & -6.8342435 \\
9 H H5 & 2.2274379 & 0.0000000 & -5.9377119 \\
10 H H4 & 2.6246014 & 0.0000000 & -3.4822174 \\
11 C C7 & 0.7045554 & 0.0000000 & -1.5578525 \\
12 H H7 & 1.7261790 & 0.0000000 & -1.1601745 \\
13 C C8 & -0.3796842 & 0.0000000 & -0.7455194 \\
14 C C9 & -1.7330331 & 0.0000000 & -1.3396594 \\
15 O O1 & -1.9039649 & 0.0000000 & -2.7101632 \\
16 O O2 & -2.8281232 & 0.0000000 & -0.8241290 \\
17 C C10 & -0.2663860 & 0.0000000 & 0.6584845 \\
18 C C11 & -0.1747357 & 0.0000000 & 1.8503971 \\
19 C C12 & -0.0763243 & 0.0000000 & 3.2617537 \\
20 C C13 & 0.1100179 & 0.0000000 & 6.0380631 \\
21 C C14 & -1.2399629 & 0.0000000 & 4.0392197 \\
22 C C15 & 1.1802958 & 0.0000000 & 3.8772385 \\
23 C C16 & 1.2664305 & 0.0000000 & 5.2643942 \\
24 C C17 & -1.1393608 & 0.0000000 & 5.4255061 \\
25 H H2 & -2.2227436 & 0.0000000 & 3.5547655 \\
26 H H3 & 2.0899818 & 0.0000000 & 3.2677744 \\
27 H H8 & 2.2483986 & 0.0000000 & 5.7488496 \\
28 H H9 & -2.0480416 & 0.0000000 & 6.0366762 \\
29 H H10 & 0.1833287 & 0.0000000 & 7.1305318
\end{tabular}




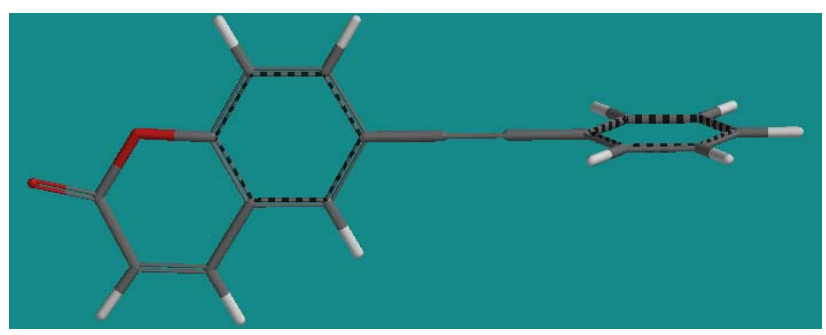

Optimized ground state structure of $\mathbf{6}$

Energy: $37.23 \mathrm{kcal} / \mathrm{mol} ; \mu=4.63 \mathrm{D} ;$ Pt.gp:Cs

\begin{tabular}{|c|c|c|c|}
\hline & \multicolumn{3}{|c|}{ Cartesian Coordinates (Angstroms) } \\
\hline & Atom & Y & Z \\
\hline $1 \mathrm{H} \mathrm{H} 1$ & -4.3396197 & 0.0000000 & \\
\hline $2 \mathrm{C} \mathrm{C} 1$ & -3.3127239 & 0.0000000 & 1.6363544 \\
\hline $3 \mathrm{C} \mathrm{C} 4$ & -0.6848425 & 0.0000000 & -1.6447841 \\
\hline $4 \mathrm{C} \mathrm{C} 2$ & -3.0751521 & 0.0000000 & -2.0212267 \\
\hline $5 \mathrm{CC}$ & 4047 & & +133 \\
\hline $6 \mathrm{CC5}$ & 7997 & 0.000 & 4624 \\
\hline $7 \mathrm{C} \mathrm{C} 3$ & 2256 & 0.000 & 13691 \\
\hline $8 \mathrm{H} \mathrm{H} 4$ & 7985 & & 17295 \\
\hline $9 \mathrm{CC} 7$ & 386 & & 9514 \\
\hline $10 \mathrm{H} \mathrm{H} 7$ & 5132 & & 05122 \\
\hline 11 C C8 & 7677 & & 91978 \\
\hline $12 \mathrm{H} \mathrm{H} 3$ & 48397 & & 716413 \\
\hline 13 C C9 & 74381 & 0.0 & 964392 \\
\hline 14001 & -5.0812964 & 0.00 & 373830 \\
\hline $150 \quad 02$ & -4.1879404 & 0.00 & -2.8287238 \\
\hline $16 \mathrm{H} \mathrm{H} 15$ & 2553 & 0.00 & 1.311685 \\
\hline 17 C C10 & 9447 & 0.0 & 0.6295463 \\
\hline 18 C C11 & 1.0923065 & 0.0 & 1.3914736 \\
\hline 19 C C12 & 2.1836893 & 0.0 & 41185 \\
\hline $20 \mathrm{C} C 13$ & & & 61150 \\
\hline 1 C C14 & 260 & -1.2 & $964 \varepsilon$ \\
\hline 22 C C 15 & & & 964 \\
\hline $23 \mathrm{C} C 16$ & & & 3954 \\
\hline $24 \mathrm{C} \quad \mathrm{C} 17$ & & & 39548 \\
\hline $25 \mathrm{H} \mathrm{H} 2$ & 86859 & -2.160 & 08596 \\
\hline $26 \mathrm{H} \mathrm{H} 6$ & 2.2986859 & 2.16066 & 2.3908596 \\
\hline $27 \mathrm{H} \mathrm{H} 5$ & 4.2163071 & 2.15298 & 3.9738156 \\
\hline $28 \mathrm{H} \mathrm{H} 8$ & 4.2163071 & -2.1529858 & 3.9738156 \\
\hline $29 \mathrm{H} \mathrm{H} 9$ & 5.1736545 & 0.0000000 & 4.7630895 \\
\hline
\end{tabular}


Cartesian Coordinates (Angstroms)

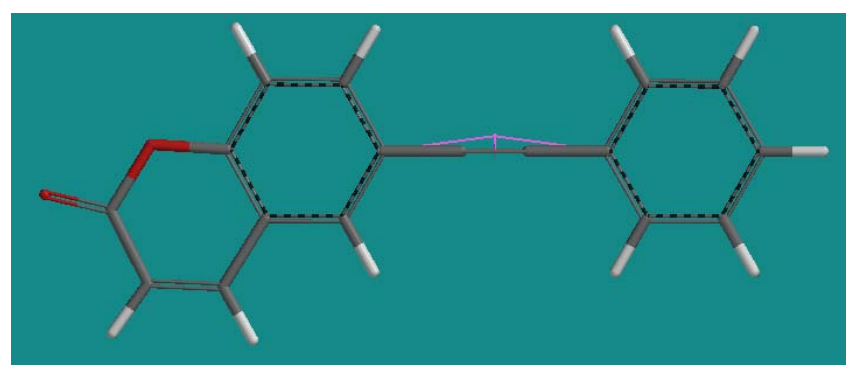

Minimum energy structure of $\mathbf{6}$ after imposing dihedral constraint of $0^{\circ}$.

Energy: $37.01 \mathrm{kcal} / \mathrm{mol} ; \mu=4.63 \mathrm{D}$; Pt.gp.: Cs

$\begin{array}{rrrrr} & \text { Atom } & \text { X } & Y & Z \\ \text { 1 H H1 } & -2.9001522 & 0.0000000 & -3.2363120 \\ \text { 2 C C1 } & -1.9006250 & 0.0000000 & -2.7854425 \\ \text { 3 C C4 } & 0.6747958 & 0.0000000 & -1.6495459 \\ \text { 4 C C2 } & -0.7561977 & 0.0000000 & -3.6007996 \\ \text { 5 C C6 } & -1.7441068 & 0.0000000 & -1.4114467 \\ \text { 6 C C5 } & -0.4582283 & 0.0000000 & -0.8400404 \\ \text { 7 C C3 } & 0.5342305 & 0.0000000 & -3.0425910 \\ \text { 8 H H4 } & 1.6766230 & 0.0000000 & -1.2043996 \\ \text { 9 C C7 } & 1.6802108 & 0.0000000 & -3.9278317 \\ \text { 10 H H7 } & 2.6821709 & 0.0000000 & -3.4835064 \\ \text { 11 C C8 } & 1.4888636 & 0.0000000 & -5.2581857 \\ \text { 12 H H3 } & 2.3117557 & 0.0000000 & -5.9806171 \\ \text { 13 C C9 } & 0.1219187 & 0.0000000 & -5.7942032 \\ \text { 14 O O1 } & -0.2660688 & 0.0000000 & -6.9417107 \\ \text { 15 O O2 } & -0.9769119 & 0.0000000 & -4.9578166 \\ \text { 16 H H15 } & -2.6251205 & 0.0000000 & -0.7597820 \\ \text { 17 C C10 } & -0.3201257 & 0.0000000 & 0.5674988 \\ \text { 18 C C11 } & -0.2045466 & 0.0000000 & 1.7570521 \\ \text { 19 C C12 } & -0.0676894 & 0.0000000 & 3.1656042 \\ \text { 20 C C13 } & 0.2020284 & 0.0000000 & 5.9356325 \\ \text { 21 C C14 } & -1.2067250 & 0.0000000 & 3.9783627 \\ \text { 22 C C15 } & 1.2067382 & 0.0000000 & 3.7434880 \\ \text { 23 C C16 } & 1.3345882 & 0.0000000 & 5.1272993 \\ \text { 24 C C17 } & -1.0651412 & 0.0000000 & 5.3608855 \\ \text { 25 H H2 } & -2.2037160 & 0.0000000 & 3.5253046 \\ \text { 26 H H6 } & 2.0974847 & 0.0000000 & 3.1065711 \\ \text { 27 H H5 } & 2.3307468 & 0.0000000 & 5.5819500 \\ \text { 28 H H8 } & -1.9549073 & 0.0000000 & 5.9991622 \\ \text { 29 H H9 } & 0.3081441 & 0.0000000 & 7.0254081\end{array}$


Cartesian Coordinates (Angstroms)

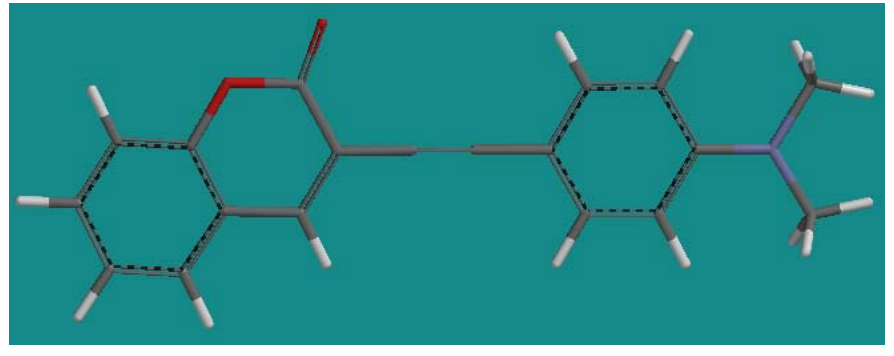

Optimized ground state geometry of $\mathbf{5}$.

Energy: $38.904 \mathrm{kcal} / \mathrm{mol}$
Atom

$1 \mathrm{H} \mathrm{H} 1$

$2 \mathrm{C}$ C1

$3 \mathrm{C}$ C4

$4 \mathrm{C} \mathrm{C} 2$

$5 \mathrm{C} \mathrm{C} 6$

$6 \mathrm{C}$ C5

$7 \mathrm{C} \mathrm{C} 3$

$8 \mathrm{H} \mathrm{H} 6$

$9 \mathrm{H} \mathrm{H} 5$

$10 \mathrm{H} \mathrm{H} 4$

$11 \mathrm{C} \mathrm{C7}$

$12 \mathrm{H} \mathrm{H} 7$

$13 \mathrm{C} \mathrm{C} 8$

14 C C9

15001

16002

17 C C10

18 C C11

19 C C12

$20 \mathrm{C} \mathrm{C} 13$

$21 \mathrm{C} \mathrm{C} 14$

22 C C15

23 C C16

$24 \mathrm{C} \mathrm{C} 17$

$25 \mathrm{H} \mathrm{H} 2$

$26 \mathrm{H} \mathrm{H} 3$

$27 \mathrm{H} \mathrm{H} 8$

$28 \mathrm{H} \mathrm{H} 9$

$29 \mathrm{~N} \mathrm{~N} 1$

30 C C18

$31 \mathrm{H} \mathrm{H} 11$

$32 \mathrm{H} \mathrm{H} 12$

$33 \mathrm{H} \mathrm{H} 13$

34 C C19

$35 \mathrm{H} \mathrm{H} 10$

$36 \mathrm{H} \mathrm{H} 14$

$37 \mathrm{H} \mathrm{H} 15$
$X$

$-7.2181135$

$-6.7666982$

$-5.6014556$

$-5.3701886$

$-7.5561708$

$-6.9772793$

$-4.7758403$

$-8.6470359$

$-7.6207629$

$-5.1472601$

$-3.3358398$

$-2.8787095$

$-2.5853073$

$-3.2546949$

$-2.8034613$

$-4.6333768$

$-1.1784327$

0.0160483

1.4268028

4.2286855

2.1846268

2.0745420

3.4582213

3.5681648

1.6789793

1.4835825

3.9321485

4.1305187

5.6425642

6.4633061

6.2655311

6.2655311

7.5285248

6.3538903

7.4386639

6.1051477

6.1051477
$Y$

$\begin{array}{rr}0.0000000 & -1.7968505 \\ 0.0000000 & -0.7982725 \\ 0.0000000 & 1.7581325 \\ 0.0000000 & -0.6489788 \\ 0.0000000 & 0.3387974 \\ 0.0000000 & 1.6120821 \\ 0.0000000 & 0.6245499 \\ 0.0000000 & 0.2422004 \\ 0.0000000 & 2.4982116 \\ 0.0000000 & 2.7558614 \\ 0.0000000 & 0.7286656 \\ 0.0000000 & 1.7249572 \\ 0.0000000 & -0.3991059 \\ 0.0000000 & -1.7163172 \\ 0.0000000 & -2.8394412 \\ 0.0000000 & -1.8095068 \\ 0.0000000 & -0.3522893 \\ 0.0000000 & -0.2953228 \\ 0.0000000 & -0.2267420 \\ 0.0000000 & -0.0983986 \\ 0.0000000 & -1.4030772 \\ 0.0000000 & 1.0132261 \\ 0.0000000 & 1.0808668 \\ 0.0000000 & -1.3428297 \\ 0.0000000 & -2.3753910 \\ 0.0000000 & 1.9355442 \\ 0.0000000 & 2.0697254 \\ 0.0000000 & -2.2841613 \\ 0.0000000 & -0.0353715 \\ 0.0000000 & -1.2570155 \\ 0.8906926 & -1.8713036 \\ -0.8906926 & -1.8713036 \\ 0.0000000 & -0.9850341 \\ 0.0000000 & 1.2528009 \\ 0.0000000 & 1.0739122 \\ -0.8904675 & 1.8485897 \\ 0.8904675 & 1.8485897\end{array}$

S51 
Cartesian Coordinates (Angstroms)

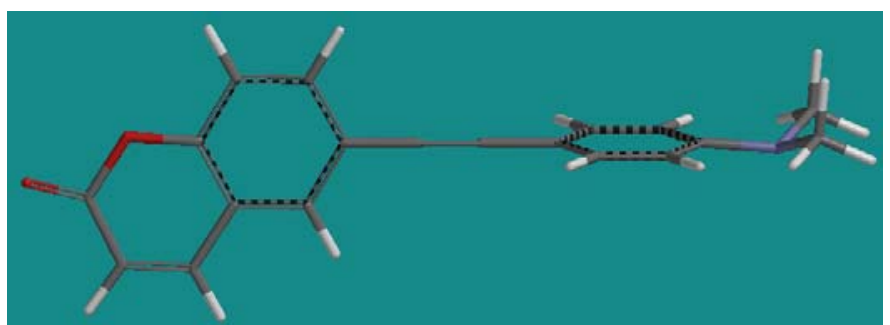

Optimized ground state geometry of 10 .

Energy: $33.48 \mathrm{kcal} / \mathrm{mol} ; \mu=5.61 \mathrm{D} ;$ Pt.gp: Cs

\begin{tabular}{lrrrr} 
& \multicolumn{1}{c}{ Atom } & X & \multicolumn{1}{c}{ Y } & \multicolumn{1}{c}{ Z } \\
\hline 1 H H1 & 2.8608857 & 0.0000000 & -5.0593671 \\
2 C C1 & 1.8653927 & 0.0000000 & -4.5998470 \\
3 C C4 & -0.6993599 & 0.0000000 & -3.4406513 \\
4 C C2 & 0.7139056 & 0.0000000 & -5.4048193 \\
5 C C6 & 1.7210308 & 0.0000000 & -3.2243075 \\
6 C C5 & 0.4406313 & 0.0000000 & -2.6415537 \\
7 C C3 & -0.5712939 & 0.0000000 & -4.8351390 \\
8 H H4 & -1.6972043 & 0.0000000 & -2.9865413 \\
9 C C7 & -1.7251349 & 0.0000000 & -5.7099058 \\
10 H H7 & -2.7229748 & 0.0000000 & -5.2564469 \\
11 C C8 & -1.5458009 & 0.0000000 & -7.0419907 \\
12 H H3 & -2.3750415 & 0.0000000 & -7.7570365 \\
13 C C9 & -0.1837467 & 0.0000000 & -7.5900597 \\
14 O O1 & 0.1937181 & 0.0000000 & -8.7413971 \\
15 O O2 & 0.9224728 & 0.0000000 & -6.7640769 \\
16 H H15 & 2.6077569 & 0.0000000 & -2.5804129 \\
17 C C10 & 0.3146516 & 0.0000000 & -1.2319555 \\
18 C C11 & 0.2085962 & 0.0000000 & -0.0416903 \\
19 C C12 & 0.0824045 & 0.0000000 & 1.3676750 \\
20 C C13 & -0.1595169 & 0.0000000 & 4.1605693 \\
21 C C14 & 0.0189579 & 1.2092500 & 2.0672584 \\
22 C C15 & 0.0189579 & -1.2092500 & 2.0672584 \\
23 C C16 & -0.1012469 & -1.2088709 & 3.4483596 \\
24 C C17 & -0.1012469 & 1.2088709 & 3.4483596 \\
25 H H2 & 0.0635243 & 2.1589871 & 1.5233717 \\
26 H H6 & 0.0635243 & -2.1589871 & 1.5233717 \\
27 H H5 & -0.1577475 & -2.1675834 & 3.9862436 \\
28 H H8 & -0.1577475 & 2.1675834 & 3.9862436 \\
29 N N1 & -0.3931496 & 0.0000000 & 5.5852641 \\
30 C C18 & -0.0027448 & -1.2252428 & 6.3197274 \\
31 H H9 & -0.5230378 & -1.2336918 & 7.2862514 \\
32 H H11 & 1.0795977 & -1.2907880 & 6.5083936 \\
33 H H12 & -0.3064571 & -2.1225690 & 5.7572016 \\
34 C C19 & -0.0027448 & 1.2252428 & 6.3197274 \\
35 H H10 & -0.5230378 & 1.2336918 & 7.2862514 \\
36 H H13 & -0.3064571 & 2.1225690 & 5.7572016 \\
37 H H14 & 1.0795977 & 1.2907880 & 6.5083936
\end{tabular}

S52 


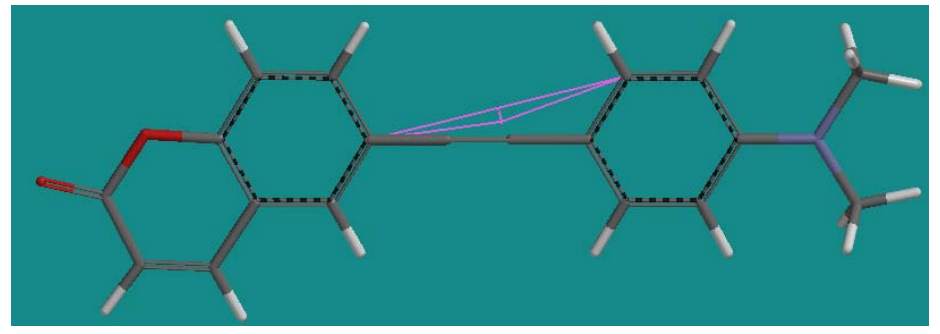

Optimized ground state geometry of $\mathbf{1 0}$ after planarization.

Energy: $37.82 \mathrm{kcal} / \mathrm{mol} ; \mathrm{m}=7.12 \mathrm{D}$; Pt. gp.: Cs
Cartesian Coordinates (Angstroms)

\begin{tabular}{|c|c|c|c|}
\hline & - & (A) & \\
\hline & $X$ & $Y$ & Z \\
\hline $1 \mathrm{H} \mathrm{H} 1$ & 2.8629458 & 0.0000000 & -5.1123388 \\
\hline 2 C C1 & 1.8755643 & 0.0000000 & -4.6356827 \\
\hline 3 C C4 & -0.6689360 & 0.0000000 & -3.4328380 \\
\hline $4 \mathrm{C} \mathrm{C} 2$ & 0.7104372 & 0.0000000 & -5.4208744 \\
\hline 5 C C6 & 1.7548656 & 0.0000000 & -3.2579783 \\
\hline 6 C C5 & 0.4845535 & 0.0000000 & -2.6525475 \\
\hline 7 C C3 & -0.5647699 & 0.000 & -4.8292499 \\
\hline $8 \mathrm{H} \mathrm{H} 4$ & -1.6587409 & 0.0000000 & -2.9617387 \\
\hline 9 C C7 & -1.7332356 & 0.0000000 & -5.6842598 \\
\hline $10 \mathrm{H} \mathrm{H} 7$ & -2.7232542 & 0.0000000 & -5.2139841 \\
\hline $11 \mathrm{C}$ C8 & -1.5767176 & & -7.0192728 \\
\hline $12 \mathrm{H} \mathrm{H} 3$ & 30741 & 000 & 99824 \\
\hline $13 \mathrm{C}$ C9 & -0.2242096 & 0.0000000 & -7.5904635 \\
\hline 14001 & 0.8958015 & 0.0000000 & -6.7839137 \\
\hline $150 \quad 02$ & 0.1328885 & 0.0000000 & -8.7485060 \\
\hline $16 \mathrm{H} \mathrm{H} 16$ & 2.6524702 & 0.00 & -2.6294761 \\
\hline 17 C C10 & 2856 & & 423970 \\
\hline 18 C C11 & 0.2985019 & 0.0000000 & -0.0498828 \\
\hline 19 C C12 & 0.1978431 & 0.0000000 & 1.3595574 \\
\hline $20 \mathrm{C} \mathrm{C} 13$ & -0.0028028 & 0.0000000 & 4.1590067 \\
\hline 21 C C14 & & 0.00 & 2.1485755 \\
\hline 22 C C15 & 216 & & 57335 \\
\hline 23 C C16 & -1.1609150 & 0.00 & 3.3571623 \\
\hline $24 \mathrm{C} \quad \mathrm{C} 17$ & 1.2577539 & 0.0000000 & 3.5305552 \\
\hline $25 \mathrm{H} \mathrm{H} 2$ & 2.3389528 & 0.0000000 & 1.6709333 \\
\hline $26 \mathrm{H} \mathrm{H} 6$ & -1.9656193 & 0.0000000 & 1.3623447 \\
\hline $27 \mathrm{H} \mathrm{H} 5$ & -2.1612155 & 0.00 & 3.8061200 \\
\hline $28 \mathrm{H} \mathrm{H} 8$ & & & 463 \\
\hline $29 \mathrm{~N} \mathrm{~N} 1$ & -0.1041464 & 0.0000000 & 5.5709271 \\
\hline 30 C C18 & 1.0946431 & 0.0000000 & 6.4244471 \\
\hline $31 \mathrm{H} \mathrm{H} 9$ & 0.7946361 & 0.0000000 & 7.4818949 \\
\hline $32 \mathrm{H} \mathrm{H} 11$ & 1.7144821 & 0.8905913 & 6.2440774 \\
\hline $33 \mathrm{H} \mathrm{H} 12$ & & & 6.2440774 \\
\hline 34 C C19 & -1.4126346 & 0.0000000 & 6.2442753 \\
\hline $35 \mathrm{H} \mathrm{H} 10$ & -1.2669760 & 0.0000000 & 7.3338046 \\
\hline $36 \mathrm{H} \mathrm{H} 13$ & -2.0003290 & -0.8905816 & 5.9771206 \\
\hline $37 \mathrm{H} \mathrm{H} 14$ & -2.0003290 & 0.8905816 & 5.91 \\
\hline
\end{tabular}


HOMO level of 1

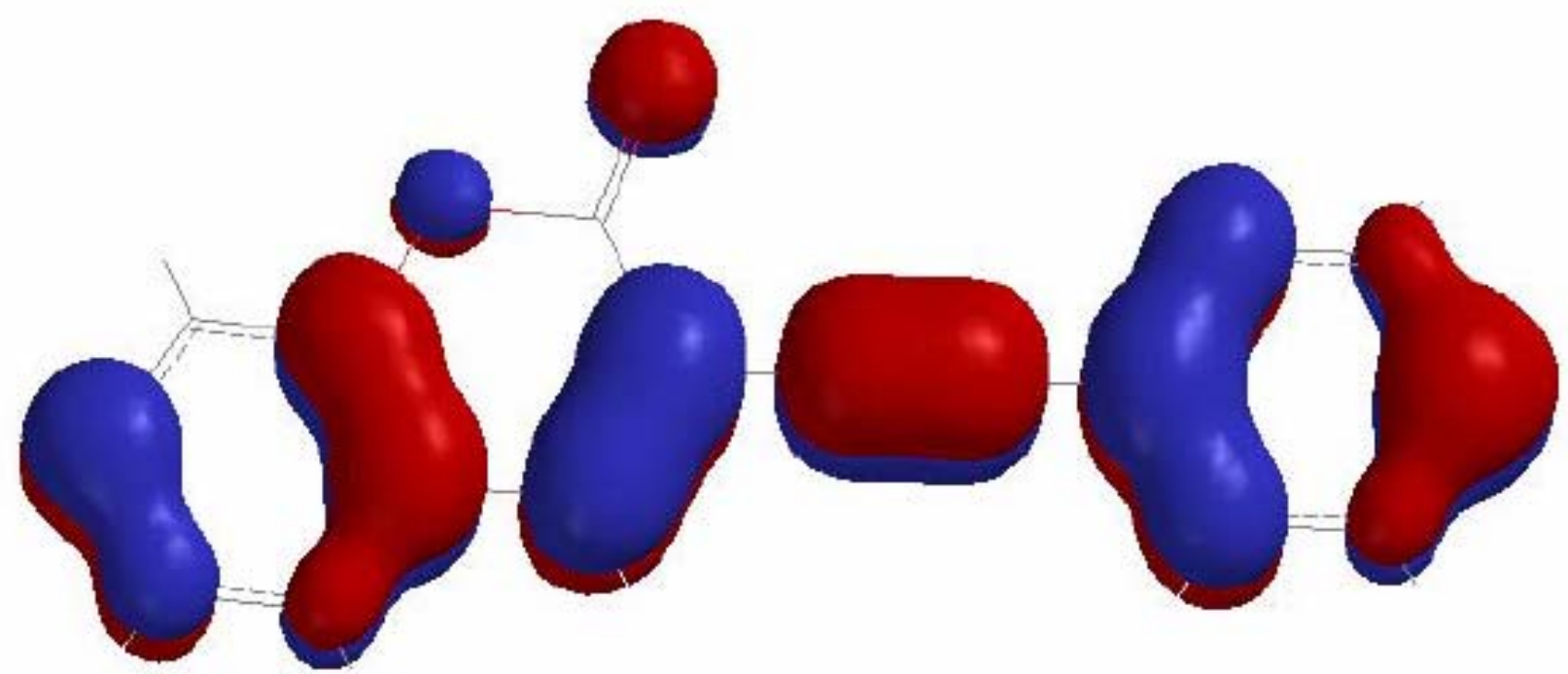

LUMO level of 1

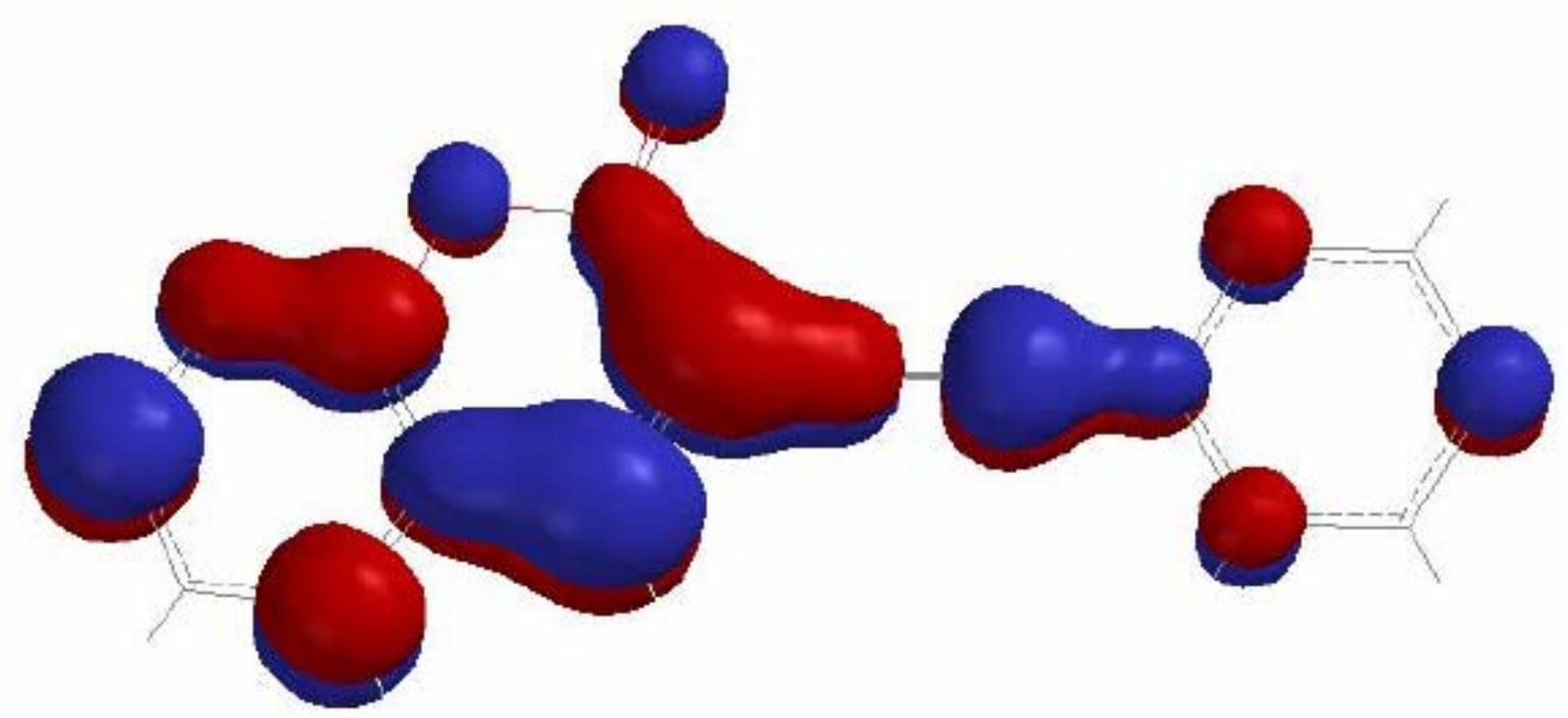


HOMO level of 6

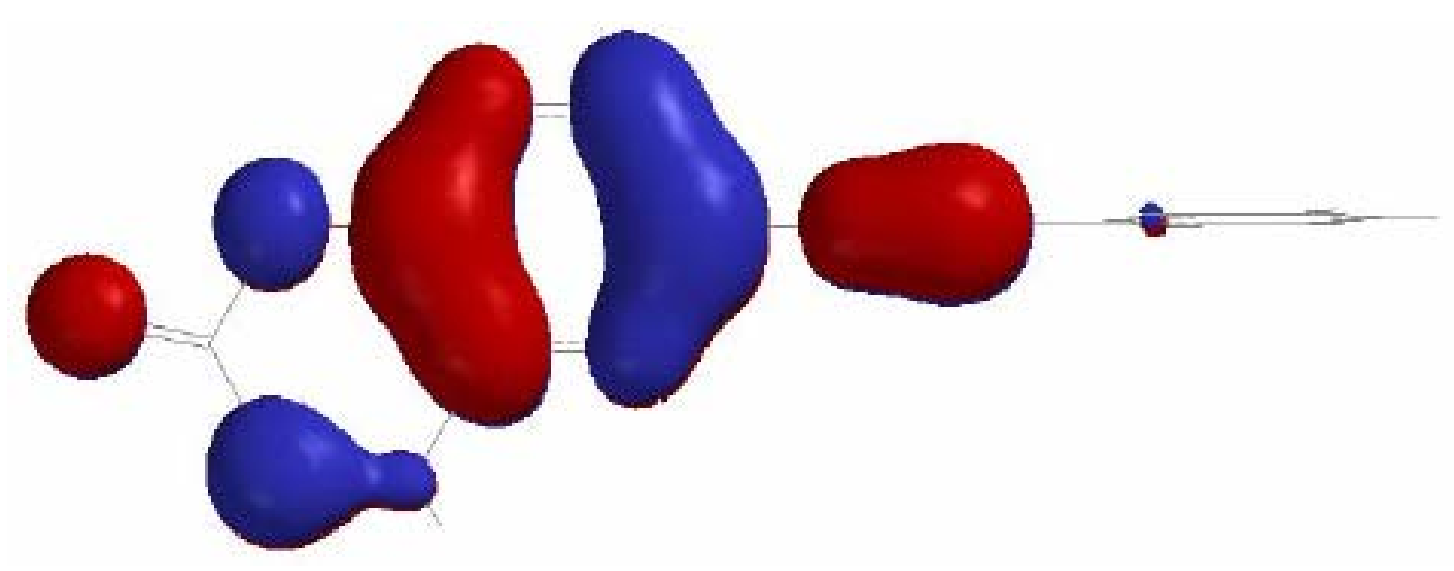

LUMO level of 6

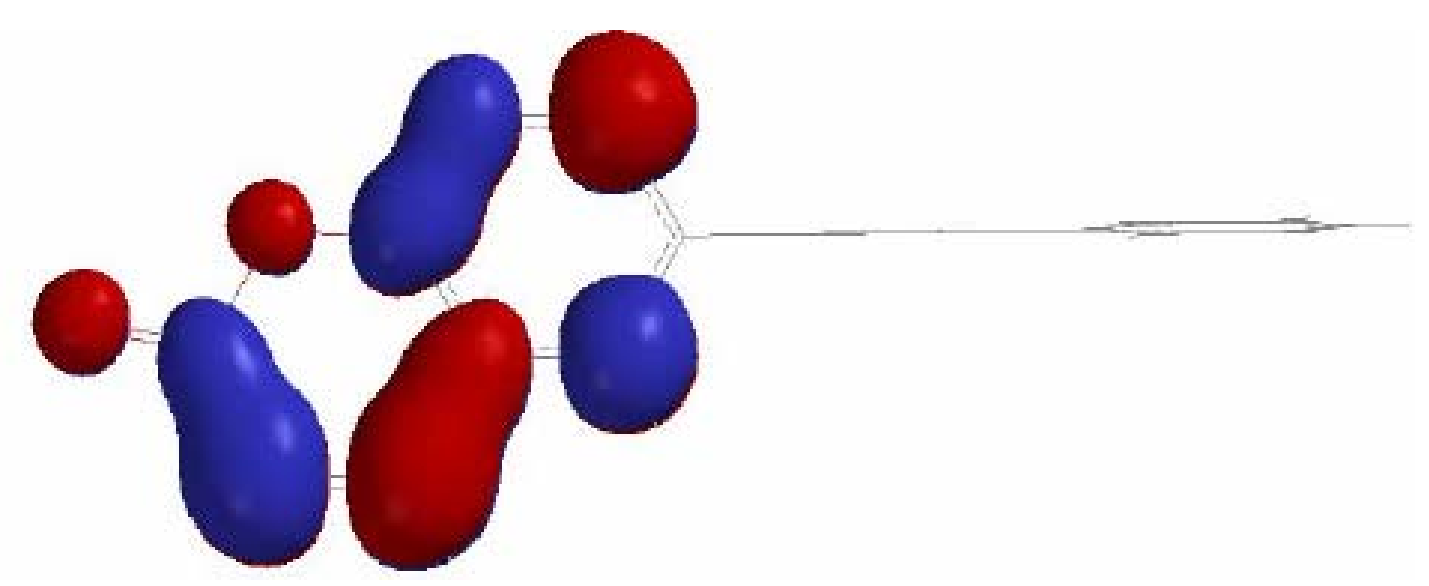


HOMO level of 6 after planarization

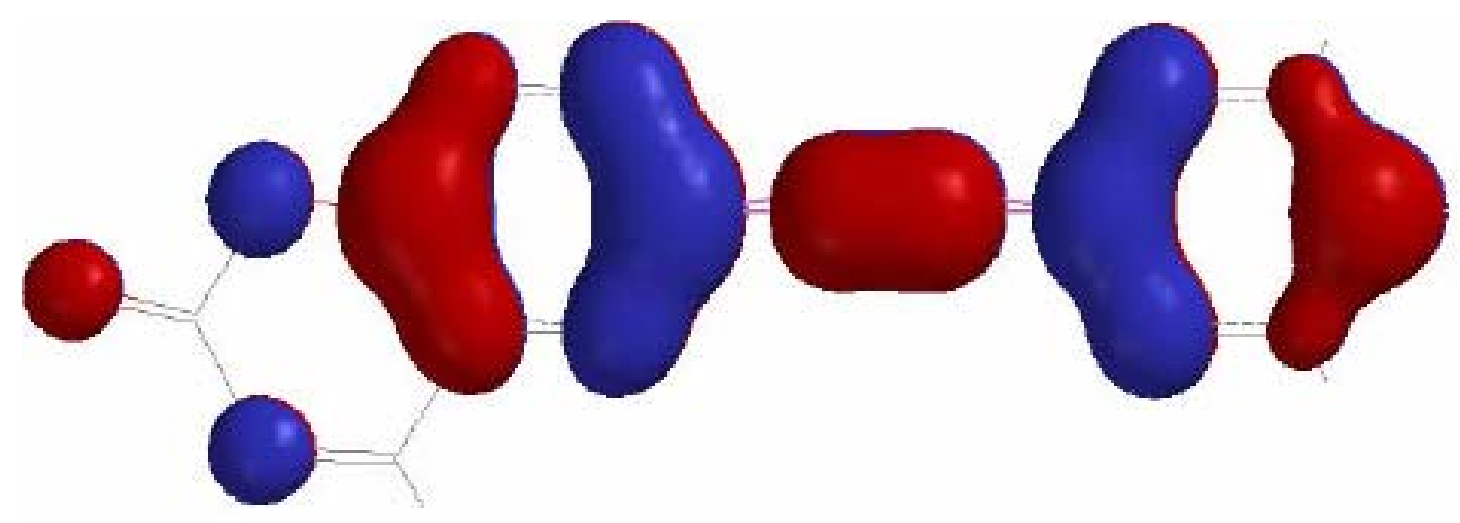

LUMO level of 6 after planarization

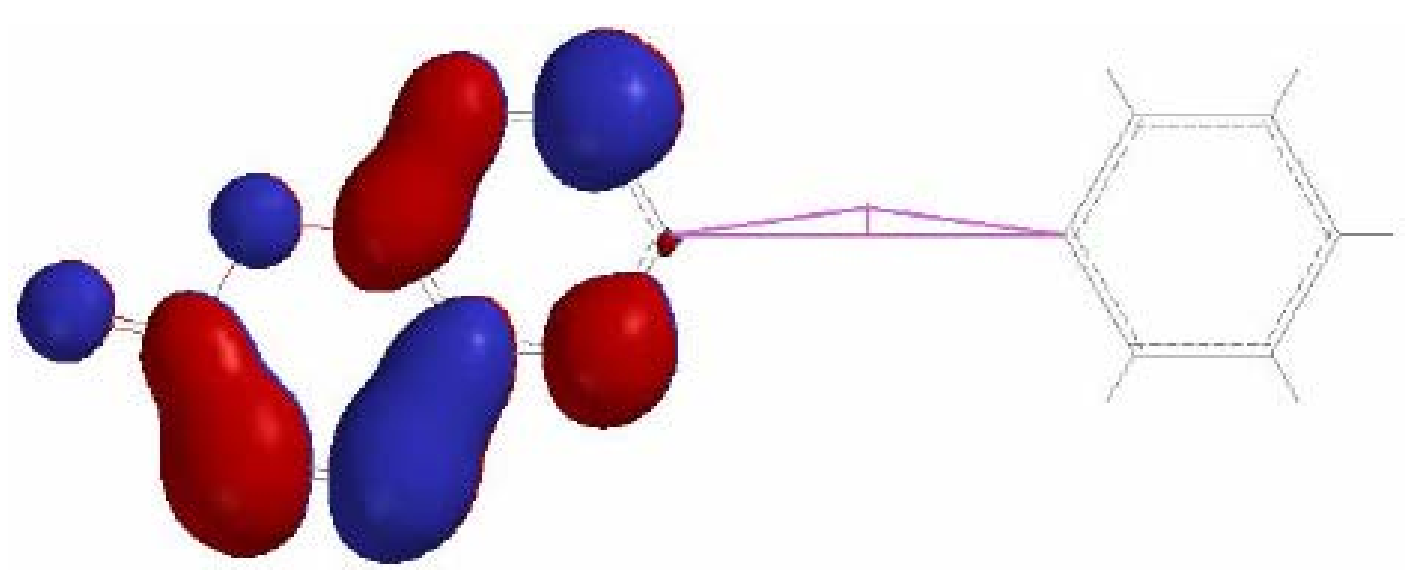


HOMO level of $\mathbf{5}$

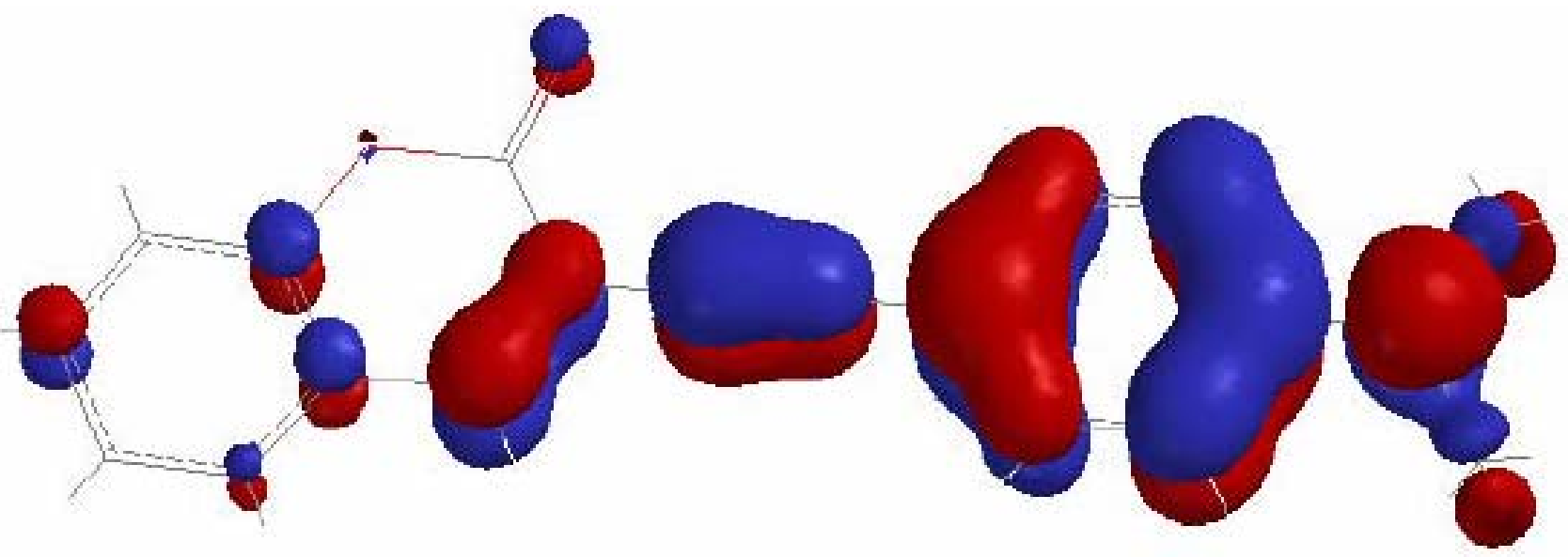

LUMO level of $\mathbf{5}$

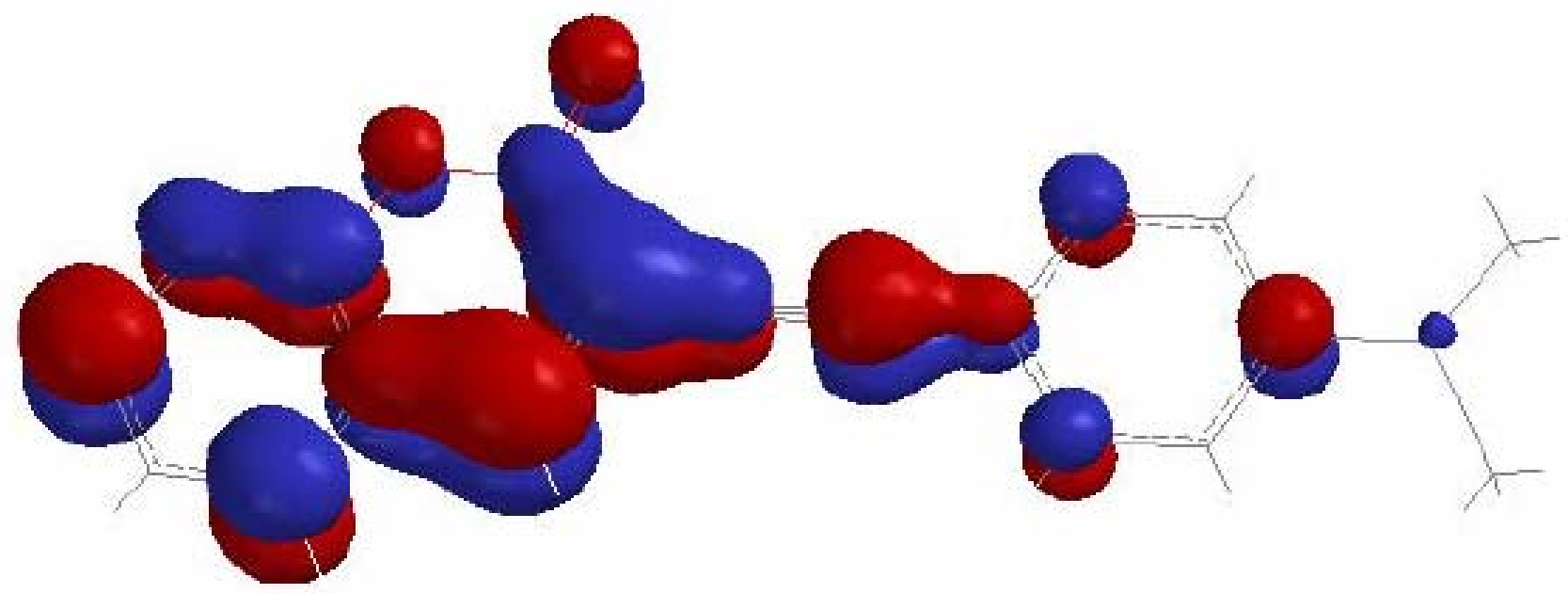


HOMO level of 10

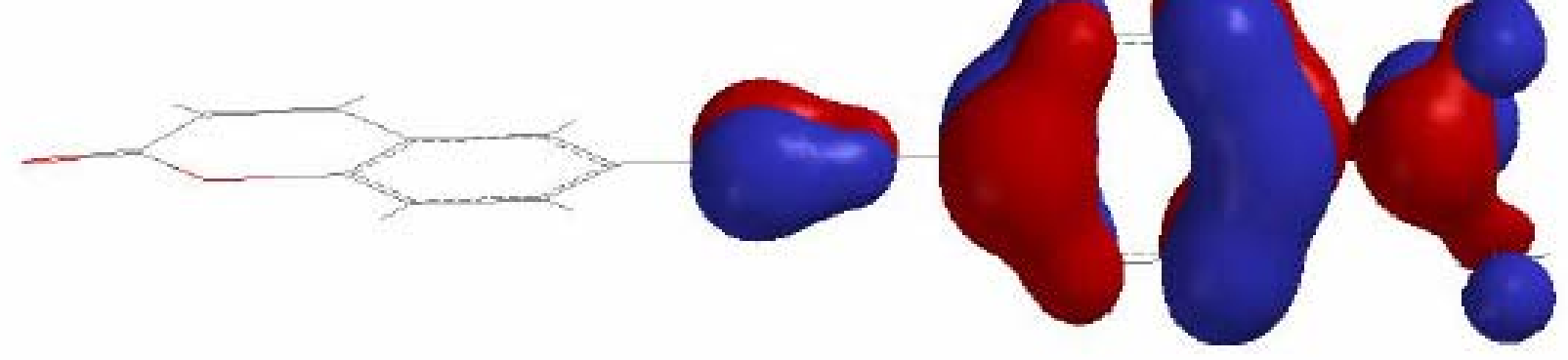

LUMO level of 10

S58 
HOMO level of 10 after planarization

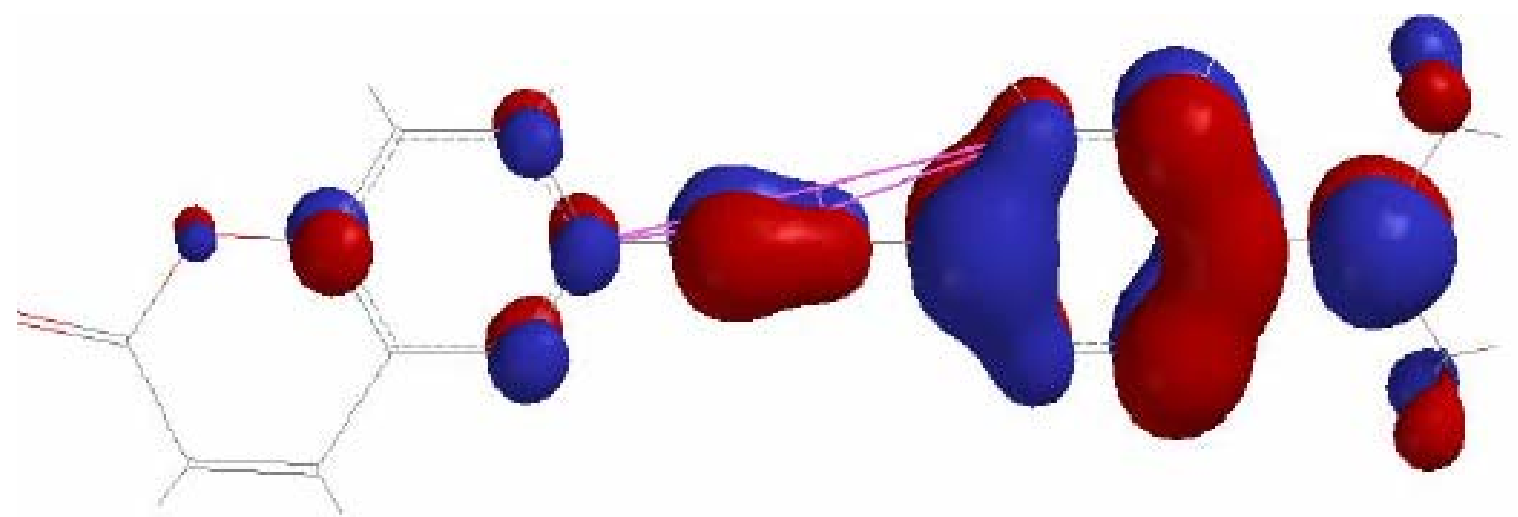

LUMO level of 10 after planarization

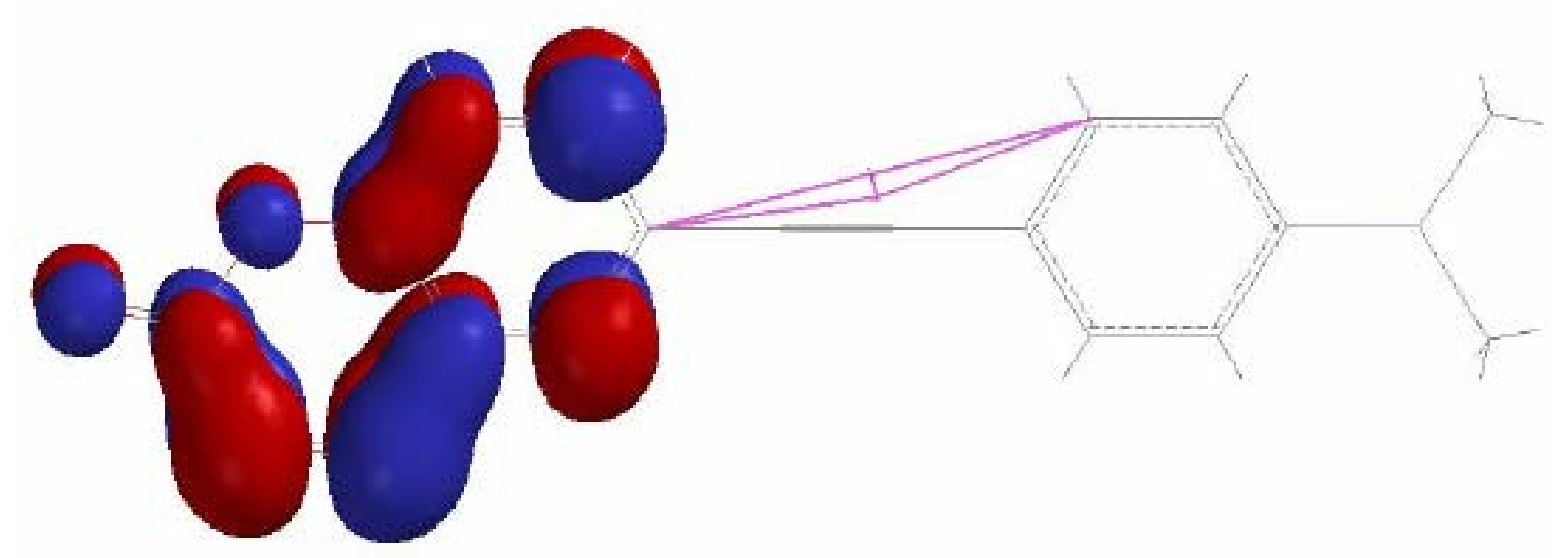

